\title{
Redox imbalance and oxidative stress in Mecp2 deficient neurons
}

\author{
Dissertation \\ for the award of the degree \\ "Doctor rerum naturalium" \\ Division of Mathematics and Natural Sciences \\ Of the Georg-August-Universität Göttingen
}

within the doctoral program Molecular Physiology of the Brain of the Georg-August University School of Science (GAUSS)

\author{
submitted by \\ Karolina Can \\ from \\ Bielawa, Poland
}

Göttingen, 2016 


\section{Thesis Committee}

Prof. Dr. Michael Müller

Universitätsmedizin Göttingen, Georg-August-Universität Göttingen, Zentrum für Physiologie und Pathophysiologie, Institut für Neuro- und Sinnesphysiologie

\section{Prof. Dr. Dr. Detlev Schild}

Universitätsmedizin Göttingen, Georg-August-Universität Göttingen, Zentrum für Physiologie und Pathophysiologie, Abtl. Neurophysiologie und Zelluläre Biophysik

\section{Dr. Sebastian Kügler}

Universitätsmedizin Göttingen, Georg-August-Universität Göttingen, Abtl. Neurologie

\section{Examination Board}

\section{Prof. Dr. Dr. Hannelore Ehrenreich}

Max-Planck-Institut für experimentelle Medizin, Klinische

Neurowissenschaften, Göttingen

Prof. Dr. Michael Hörner

Johann-Friedrich-Blumenbach-Institut für Zoologie und Anthropologie Abt. Zelluläre Neurobiologie, c/o ENI, Göttingen

\section{Prof. Dr. Thomas Dresbach}

Universitätsmedizin Göttingen, Zentrum Anatomie, Abt. Anatomie und Embryologie

Date of oral examination: 05.09.2016 
Men love to wonder, and that is the seed of our science.

Ralph Waldo Emerson 


\section{Declaration}

I hereby declare that my doctoral thesis "Redox imbalance and oxidative stress in Mecp2 deficient neurons" has been written independently with no other sources and aids than quoted. This thesis has not been submitted elsewhere for any academic degree.

Göttingen, 18.052016

KAROLINA CAN 


\section{Contents}

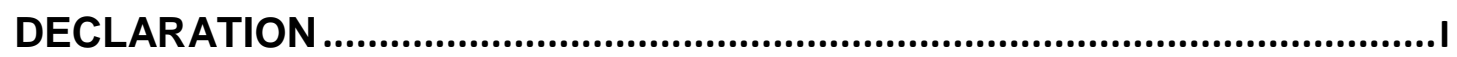

LIST OF ABBREVIATIONS ........................................................................

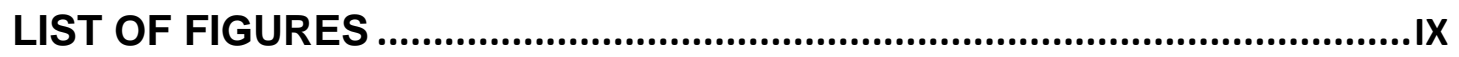

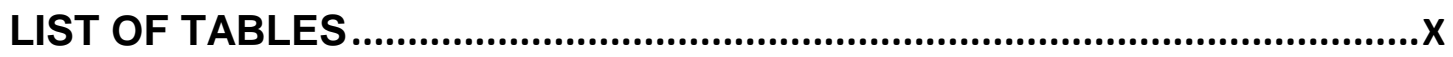

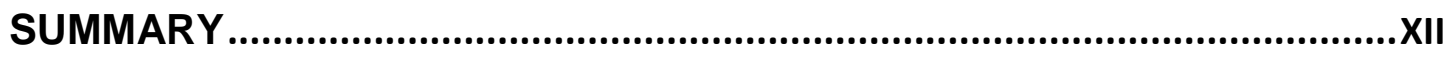

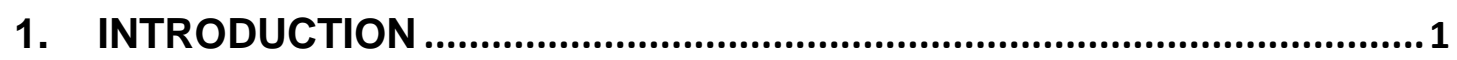

1.1. Pathology of Rett syndrome ……............................................................

1.2. Genetic cause of Rett syndrome .............................................................

1.3. MeCP2 protein - structure, function and expression...........................

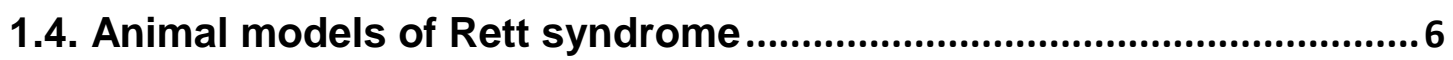

1.5. Breathing impairment and mitochondriopathy in Rett syndrome ......7

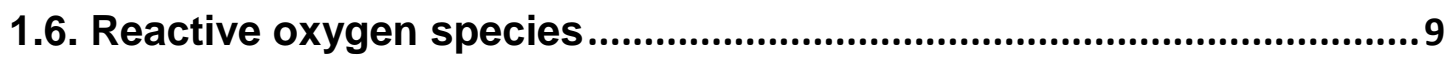

1.7. Oxidative stress in Rett syndrome and oxidative stress markers.... 12

1.8. Imaging of reactive oxygen species - outline of redox sensors ...... 14

2. AIMS OF THIS THESIS ...................................................................... 16

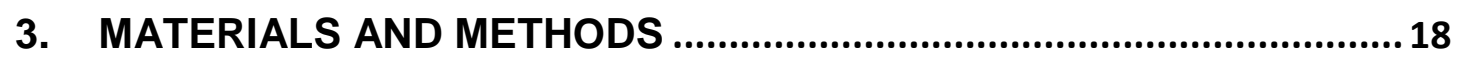

3.1. Solutions and pharmacological compounds......................................18

3.2. Mouse model of Rett syndrome............................................................. 19

3.3. Preparation of dissociated cell and organotypic cultures...................20

3.4. Culturing media .............................................................................................. 21

3.5. Preparation of brain acute slices ............................................................ 22

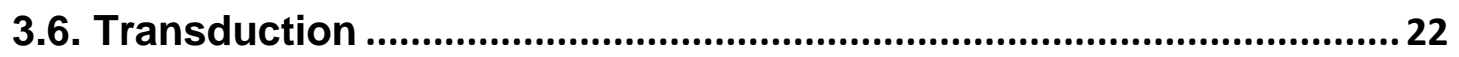

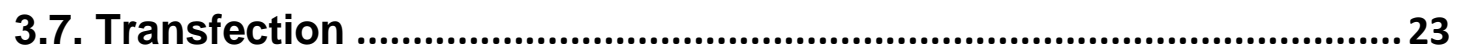


3.8. Visualizing of mitochondria in vitro using MitoTracker RED FM .....23

3.9. Immunostaining of neurons with MAP2 ….......................................24

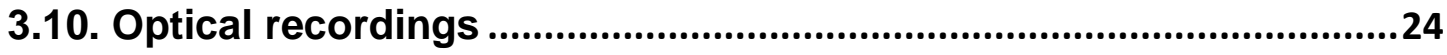

3.11. The 2-photon laser scanning microscope........................................26

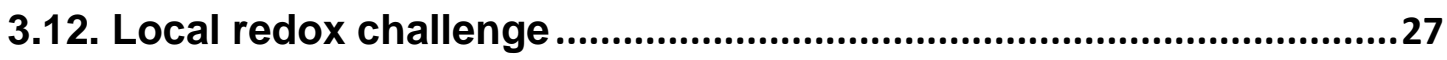

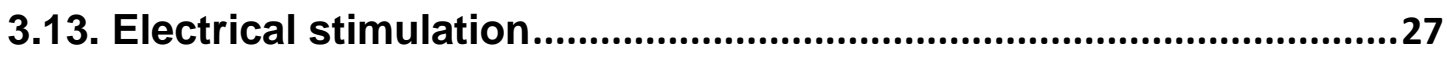

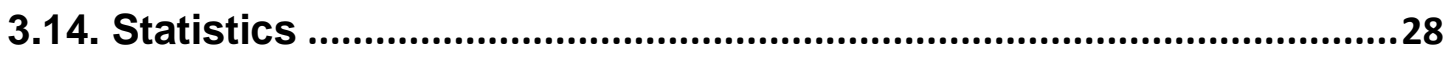

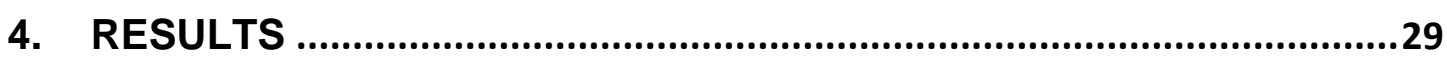

4.1. Challenge to select the ideal optical redox indicator .........................29

4.1.1. Calibration of the SypHer sensor ................................................30

4.1.2. Challenging SypHer by various redox stimuli ...............................32

4.2. Redox changes in primary neuronal cell culture and organotypic

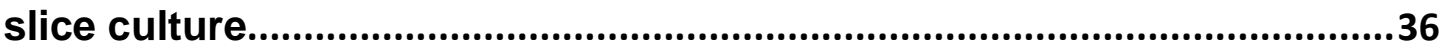

4.2.1. Confirming proper sub-cellular localization of mito- and cyto-

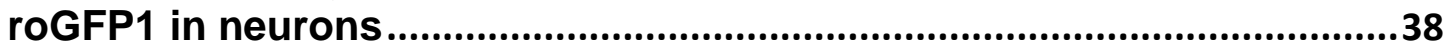

4.2.2. Calibration of cyto- and mito-roGFP1 ...........................................39

4.2.3. The roGFP1 pre-treatment baseline ratio in mitochondria and

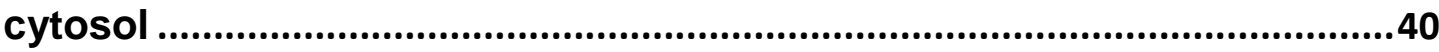

4.2.4. Mitochondrial and cytosolic redox status...................................42

4.3. Sub-cellular redox changes under control conditions and upon oxidative challenge. ............................................................................47

4.4. Local redox stimulation of primary neurons...................................50

4.5. Electrical stimulation of neurons....................................................56

4.6. The effect of neurotransmitters on cellular redox balance................60

4.7. Mechanisms of transmitter-mediated redox changes ......................62

4.8. The roGFP1-transgenic mice as a novel tool for cellular redox imaging during disease progression. .......................................................69

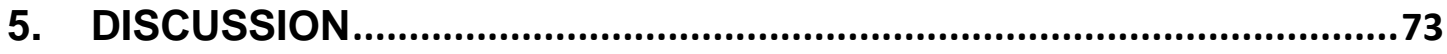


5.1. The genetically-encoded sensors allow for ratiometric measurements of redox imbalance.

5.1.1. Diminished reliability of the redox sensor HyPer

5.2. The genetically-encoded optical redox sensor roGFP1 allows for detailed and quantitative monitoring of ROS/redox changes

5.3. Redox homeostasis and mitochondrial function are impaired in Mecp2 $^{-/ y}$ neurons

5.3.1. Mecp2 $^{-/ y}$ neurons are more oxidized already under control conditions

5.3.2. Redox imbalance is more pronounced in somatic areas of hippocampal neurons 80

5.3.3. Mecp2 $^{-/ y}$ neurons reveal altered responses to redox challenge and show less efficient superoxide dismutation

5.3.4. Mecp2 $^{-/ y}$ hippocampus is more susceptible to hypoxia and mitochondrial challenge

5.4. Intact neurotransmission is required for a proper neuronal network functioning

5.4.1. Activation of NADPH oxidase and xanthine oxidase contributes to ROS production in response to glutamate.

5.4.2. Dopamine modulates mitochondrial function in $\mathbf{M e c p 2}^{-/ y}$ hippocampal neurons

6. Redox imbalance occurs not only in $\mathrm{Mecp2}^{-/ y}$ neonates but also in neurons of adult and symptomatic Rett mice.

6. CONCLUSION AND FUTURE PERSPECTIVES

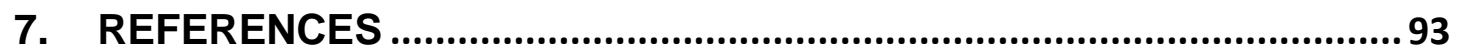

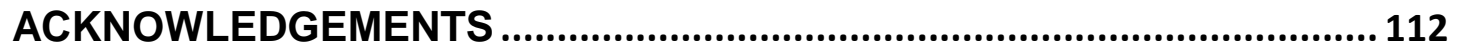

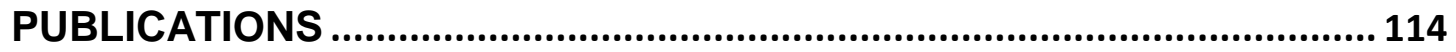

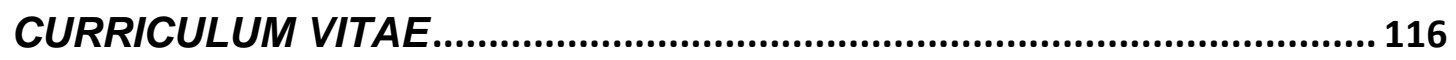




\section{List of abbreviations}

A

ACSF

Allo

AMC

AMPA

AQP8

ATP

BDNF

C

CA

CA1

CA3

$\mathrm{Ca}^{2+}$

$\mathrm{CaCl}_{2}$

cAMP

Cat

CDKL5

$\mathrm{CN}^{-}$

$\mathrm{CO}_{2}$

CpG

cPYFP

CREB1

CTD

cyto-roGFP1

Adenine

Artificial cerebrospinal fluid

Allopurinol

Antimycin A

a-amino-3-hydroxy-5-methyl-4-isoxazolepropionic acid

Aquaporin-8

Adenosinetriphosphate

Brain-derived neurotrophic factor protein (human)

Cytosine

Cornu ammonis

Cornu ammonis 1

Cornu ammonis 3

Calcium ion

Calcium chloride

Cyclic adenosine monophosphate

Catalase

Cyclin-dependent kinase-like 5 gene (human)

Cyanide

Carbon dioxide

Cytosine-phosphate-guanine

Circularly permuted yellow fluorescent protein

cAMP responsive element binding protein 1

C-terminal domain

Reduction/oxidation-sensitive green fluorescent protein 1 selectively targeting cytosol

DIV

Days in vitro

DG

Dentate gyrus

DMSO

Dimethyl sulfoxide

DNA

Deoxyribonucleic acid

DPI

Diphenyleneiodonium chloride

DTT

1,4-dithio-DL-threitol

e.g.

Exempli gratia 
FAD

FCCP

FCS

$\mathrm{Fe}^{2+}$

FOXG1

FRET

GFP

GP

GR

GSH

GSSG

GST

$\mathrm{H}_{2} \mathrm{O}$

$\mathrm{H}_{2} \mathrm{O}_{2}$

HBSS

HDAC

HEPES

HIF1a

HyPer

IGF-1

IsoPs

$\mathrm{K}^{+}$

$\mathrm{KCl}$

$\mathrm{KOH}$

LOI

MAP2

MBD

MECP2

MECP2

Mecp2

$\mathrm{MeCP} 2$

MEM

$\mathrm{Mg}^{2+}$

$\mathrm{MgCl}_{2}$
Flavin adenine dinucleotide

Carbonyl cyanide 4-trifluoromethoxy)phenylhydrazone

Fetal calf serum

Iron ion

Forkhead box protein G1 gene (human)

Fluorescence resonance energy transfer

Green fluorescent protein

Glutathione peroxidase

Glutathione reductase

Glutathione

Glutathione disulfide

Glutathione S-transferase

Water

Hydroxyl peroxide

Hanks' balanced salt solution

Histone deacetylase

4-(2-hydroxyethyl)piperazine-1-ethanesulfonic acid

Hypoxia-inducible factor 1 alpha

Hydroxyl peroxide sensor

Insuline-like growth factor 1

Prostaglandin-like-compounds

Potasium ion

Potassium chloride

Potasium hydroxide

Line of interest

Microtubule associated protein 2 neuronal marker

Methyl-CpG-binding domain

Methyl-CpG binding protein 2 protein (human)

Methyl-CpG binding protein 2 encoding gene (human)

Methyl-CpG binding protein 2 encoding gene (mouse)

Methyl- CpG binding protein 2 protein (mouse)

Minimum essential medium

Magnesium ion

Magnesium chloride 


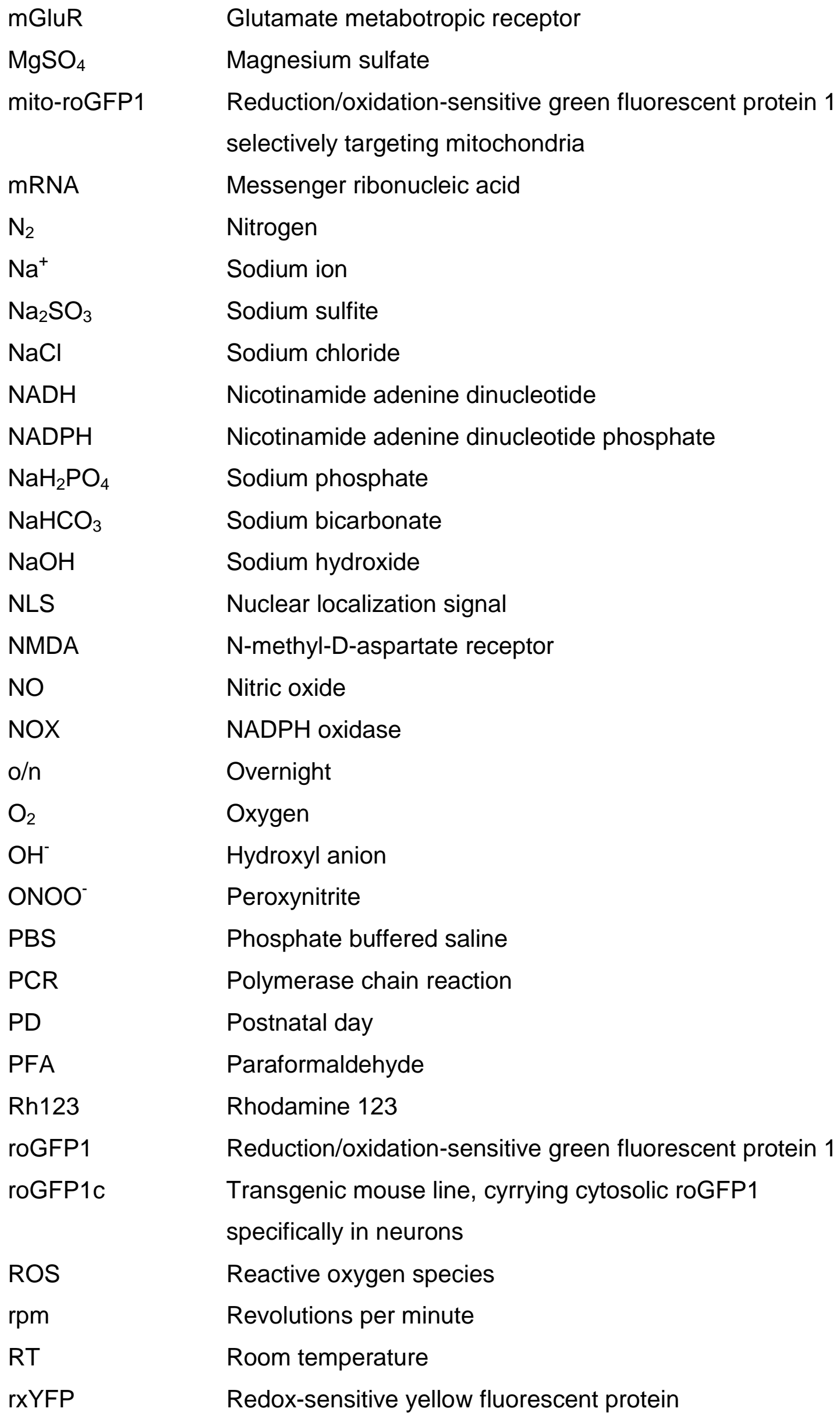


SD

$\operatorname{Sin} 3 A$

SOD

SOD1

SOD2

SOD3

S-S

SypHer

$\mathrm{T}$

tBHP

TRD

$\mathrm{TRX} 1$

TRX2

Trx

Ugcrc1

(mouse)

UTR

WT

$\mathrm{XDH}$

XO

YFP

. $\mathrm{O}_{2}{ }^{-}$

. $\mathrm{OH}$

3
Standard deviation

SIN3 transcription regulator family member $A$

Superoxide dismutase

Cu-Zn-SOD; cooper, zinc superoxide dismutase (cytosol)

Mn-SOD; manganian superoxide dismutase

(mitochondria)

EC-SOD (extracellular elements)

Disulfide bridge

Modified, $\mathrm{pH}$-sensitive and redox-insensitive variant of HyPer optical sensor (Hyper C199S)

Tymine

Tert-butylhydroperoxide

Transcription repression domain

Thioredoxin isoform 1

Thioredoxin isoform 2

Thioredoxin reductase

Ubiquinol-cytochrome c reductase core protein 1 gene

Untranslated region

Wildtype

Xanthine dehydrogenase

Xanthine oxidase

Yellow fluorescent protein

Superoxide

Hydroxyl radical

3-phosphate-end 


\section{List of figures}

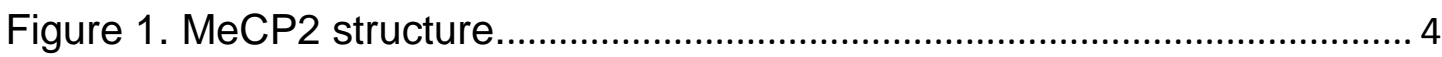

Figure 2. MeCP2 acts as a transcriptional repressor and activator.................. 5

Figure 3. ROS generation and cellular scavenging systems ............................ 10

Figure 4. ROS as a potential source of cellular damage .................................... 12

Figure 5. The 'Bird strain' Mecp2 $2^{\text {tm1.1Bird }}$ mouse model of Rett syndrome....... 19

Figure 6. Ratiometric response properties of roGFP1.................................... 26

Figure 7. Hippocampal primary cell transfected with SypHer............................ 30

Figure 8. The $\mathrm{pH}$-calibration of the SypHer sensor. ............................................. 31

Figure 9. Full range calibration of SypHer recorded as an example in a single

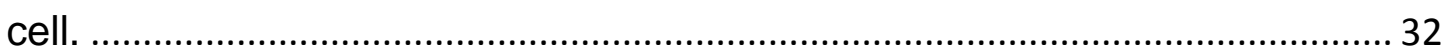

Figure 10. HyPer and SypHer responses to modulation of cellular $\mathrm{pH} . \ldots \ldots \ldots . . .33$

Figure 11. SypHer responses to redox challenge............................................... 34

Figure 12. SypHer responses to hypoxia and excitotoxicity.............................. 35

Figure 13. Summary of SypHer responses to changes in redox balance....... 35

Figure 14. Transduction of primary cell and slice cultures with cyto-roGFP1

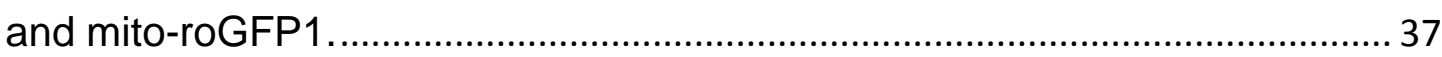

Figure 15. Sub-cellular localization of mito-roGFP1 and cyto-roGFP1 .......... 38

Figure 16. Response range of mito-roGFP1 and cyto-roGFP1 ........................ 39

Figure 17. The cytosolic and mitochondrial pre-treatment baseline ratio

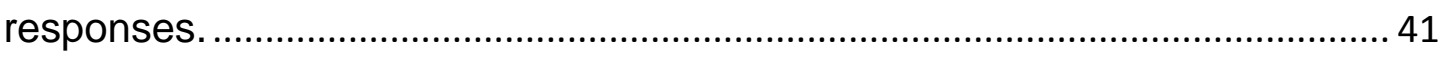

Figure 18. Rating of cellular redox homeostasis.............................................. 43

Figure 19. Summary of redox responses in mitochondria and cytosol. .......... 45

Figure 20. Oxidation level changes between somata and dendrites in WT and $M e c p 2^{-1 /}$ neurons under control conditions. ........................................................ 47

Figure 21. Local redox changes in response to oxidation challenge................ 48

Figure 22. Local neuronal changes in response to SOD inhibition................... 49

Figure 23. Rh123 release from a pipette tip by using pulsed puff stimuli. ...... 50

Figure 24. Local redox stimulation of a cyto-roGFP1-transduced neuron. ..... 51

Figure 25. Sub-cellular changes within a single neuron upon local oxidant

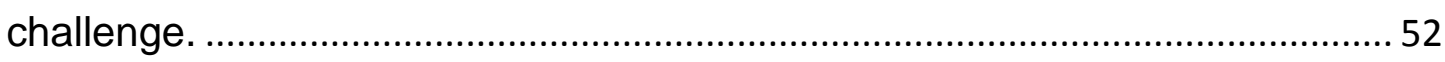

Figure 26. Local stimulation of a neuron with low doses of $\mathrm{H}_{2} \mathrm{O}_{2} \ldots \ldots \ldots \ldots \ldots \ldots \ldots . . . . .53$ 
Figure 27. Relative redox changes in dendrites and soma of neurons. .55

Figure 28. Principle of electrical stimulation.

Figure 29. Fluo-3 responses upon massive neuronal depolarisation. .58

Figure 30. The cyto-roGFP1 ratiometric response to extracellular electrical stimulation. .58

Figure 31 . Variability of cyto-roGFP1 ratio responses to extracellular electrical stimulation. .59

Figure 32. The cyto-roGFP1 ratio responses to neurotransmitters. .61

Figure 33. Mechanistic deciphering of the glutamate-mediated roGFP1

responses. .64

Figure 34. Mechanistic analysis of dopamine-mediated redox responses. ....65

Figure 35. Dual laser ratiometric 2-photon excitation of hippocampal and cortical neurons of roGFP1c transgenic Mecp2 $2^{-/ y}$ mice.

Figure 36. Redox baselines in hippocampal and cortical neurons of adult roGFP1c mice. .71

Figure 37. Redox hypothesis of Rett syndrome disease progression. .79

\section{List of tables}

Table 1. SypHer ratio changes induced by various $\mathrm{pH}$. .31

Table 2. The $\mathrm{pH}$ changes of redox-insensitive sensor SypHer. .33

Table 3. The $\mathrm{pH}$ changes of SypHer sensor upon various redox stimuli. .36

Table 4. Calibration of the roGFP1 redox sensors in mitochondrial and cytosolic compartments.

Table 5. Mito-roGFP1 and cyto-roGFP1 ratio baselines under control conditions in WT and Mecp2 $2^{-/ y}$ neurons.

Table 6. Overview of the mito-roGFP1 and cyto-roGFP1 ratio responses in cultured neurons and hippocampal organotypic slices upon various redox stimuli.

Table 7. Relative changes in the cyto-roGFP1 ratio in somatic and dendritic areas of WT and $M e c p 2^{-/ y}$ neurons upon oxidative challenge.

Table 8. Differences in oxidation levels of somatic and dendritic areas between Mecp2 $^{-/ y}$ and WT neurons in response to DEDTC. 
Table 9. Oxidative changes in dendrites and soma in response to locally stimulated neurons.

Table 10. Effects of neurotransmitters on the cyto-roGFP1 ratio in hippocampal cultured neurons.

Table 11. Redox changes in response to glutamate and dopamine upon $\mathrm{Ca}^{2+}$ depletion, mitochondrial uncoupling, NADPH oxidase inhibition and xanthine oxidation blockade.

Table 12. RoGFP1 baseline changes upon arrest of potential cellular ROS sources.

Table 13. RoGFP1 baselines of adult hippocampal and cortical neurons in acute tissue slices of roGFP1c transgenic Mecp2 $2^{-1 y}$ mice. 


\section{Summary}

Rett syndrome is a neurodevelopmental disorder that primarily occurs in girls with a prevalence of 1:10.000-1:15.000 life births. The main genetic reasons of Rett syndrome are mutations in the methyl-CpG binding protein 2 (MECP2) gene. After a short, but normal development, a Rett child falls into developmental stagnation, which is followed by neuronal and autonomic dysfunction, and manifests as mental retardation, breathing impairment, epilepsy, loss of speech, mobility disturbances and stereotypical hand movements. Growing evidence shows that Rett syndrome is associated with mitochondrial dysfunction and oxidation stress. Mitochondria of MeCP2deficient $\left(\mathrm{Mecp}^{-/ y}\right)$ mouse brain have been previously confirmed to be partly uncoupled and to show increased respiratory rates. More oxidized baseline conditions, exaggerated responses to oxidants and mitochondrial inhibition have been detected in the hippocampus of $M e c p 2^{-/ y}$ mice. To unveil the molecular causes of this redox imbalance specifically in neurons, and to enable a quantitative live-cell imaging of sub-cellular redox dynamics, viral vectors, expressing the genetically-encoded optical redox sensor reduction/oxidation-sensitive green fluorescent protein 1 (roGFP1) in cytosol and mitochondrial matrix have been generated. For quantitation, the ratiometric responses of roGFP1s were calibrated to full oxidation and reduction in mitochondrial and cytosolic compartments. Detailed fluorescence microscopy and two photon imaging confirmed that mitochondrial and cytosolic redox baselines were more oxidized in $\mathrm{Mecp2}^{-1 /}$ hippocampal neurons. Redox challenge induced by hydrogen peroxide $\left(\mathrm{H}_{2} \mathrm{O}_{2}\right)$ and severe hypoxia elicited intensified oxidizing and reducing transients in $\mathrm{Mecp}^{-/ y}$ neurons, respectively. Moreover, inhibition of superoxide dismutase (SOD) caused a less intense oxidation in $M e c p 2^{-/ y}$ cytosol and mitochondria, suggesting a decreased efficiency of this scavenging enzyme in Rett mice. Interestingly, differences among wildtype (WT) and $M e c p 2^{-1 y}$ mice were evident especially in the more complex organotypic slices, and they occurred already at neonatal stages in mitochondria and the cytosol. Furthermore, the current work is the first study, showing a pronounced shift towards more oxidizing conditions in $M e c p 2^{-/ y}$ neurons in response to different 
neurotransmitters. Taking advantage of the recently generated transgenic mouse lines, stably expressing roGFP1 in neuronal cytosol, the redox changes could be also confirmed for hippocampal neurons of adult and symptomatic $M e c p 2^{-/ y}$ mice. Taken together, roGFP1 responds reliably to oxidation and reduction, and it allows for semi-quantitative recordings of redox changes specifically in neurons. Since mitochondria are a primary source of reactive oxygen species (ROS), and the neuronal mitochondria of $M e c p 2^{-1 y}$ hippocampus revealed a more oxidizing and more vulnerable redox balance, this supports the hypothesis that mitochondrial dysfunction underlies the oxidative burden in Rett syndrome and drives potentially disease progression. Moreover, the roGFP1 transgenic mice will extend quantitative redox imaging to all postnatal stages and more complex preparations. This will enable studying disease progression and redox conditions throughout the brain of maturing Rett and WT mice. 



\section{Introduction}

\subsection{Pathology of Rett syndrome}

Rett syndrome is a progressive, postnatal and neurodevelopmental disorder which was originally described by the Austrian paediatrician Andreas Rett as an 'unusual brain atrophy syndrome' in 1966 (Rett 1966; Hagberg et al. 1983). This X-linked disorder affects predominantly girls with a prevalence of approximately 1:10.000-1:15.000 live births worldwide, and is apparently lethal in male embryos. However, individual cases of Rett syndrome in male patients were also reported (Wan et al. 1999; Clayton-Smith et al. 2000; Meloni et al. 2000; Orrico et al. 2000; Villard et al. 2000).

Four main stages constitute the typical disease progression, spanning from normal development through developmental and neuronal stagnation up to rapid regression, and final motor deterioration (Chahrour and Zoghbi 2007). Rett girls develop properly during the first 6-18 months. Hence, the first step of Rett syndrome often might be not even noticed. While fine motor functions develop, head growth and circumference starts to decelerate (Schultz et al. 1993). Along with an acquired microcephaly, general growth retardation is followed by a loss of weight and muscle hypotonia. With the onset of the rapid regression, patients start loosing hand skills, developing stereotypic hand movements such as washing movements, clapping, wringing, flapping or putting hands into the mouth (Chahrour and Zoghbi 2007; Weng et al. 2011). Furthermore, motor abnormalities together with seizures, loss of speech and social interaction occur and patients fall into a stationary, and late motor deterioration stage. They also acquire additional symptoms such as scoliosis, mental retardation, autism, epilepsy, sleep disturbances and anxiety (Chahrour and Zoghbi 2007). Other characteristic features, which might associate with the disease are teeth grinding, laughing or crying at night and screaming episodes (Young et al. 2007; Weng et al. 2011).

Furthermore, although the lifespan of Rett patients is very diverse and some individuals might even reach their 60s (Hagberg et al. 2001; Hagberg 2005), life expectancy might be highly reduced due to serious breathing 
complications, which is one of the hallmarks of Rett syndrome (Kerr et al. 1997; Julu et al. 2001; Acampa and Guideri 2006). The respiratory abnormalities appear in the rapid regression stage of the disease and include breath-holding, aerophagia, forced expulsion of air and saliva as well as frequent apnoeas (Chahrour and Zoghbi 2007).

Impaired regulation of normal brain development might arise from changes in various neurotransmitters and neuropeptides such as altered levels of dopamine (Zoghbi et al. 1985; Brucke et al. 1987; Zoghbi et al. 1989; Lekman et al. 1990; Percy 1992), serotonin (Segawa 1997), noradrenaline, acetylcholine (Wenk and Mobley 1996; Wenk 1997; Wenk and HaussWegrzyniak 1999), nerve growth factor (Lappalainen et al. 1996; Riikonen and Vanhala 1999), endorphins, substance P (Matsuishi et al. 1997; Deguchi et al. 2000), glutamate (Hamberger et al. 1992; Lappalainen and Riikonen 1996) and other aminoacids as well as their receptor expression levels (Jellinger 2003; Acampa and Guideri 2006). Altered neurotrophin signalling together with reduced serotonin plasma and substance $P$ levels may lead to disturbances in the autonomic nervous system. This causes cardiac autonomic nervous system alterations, sympathetic imbalance and reduced cardiac vagal tone, each of which contributes to life threatening cardiac arrhythmias. In fact, sudden death represents $\sim 25 \%$ of mortality rate among Rett patients (Kerr et al. 1997; Julu et al. 2001; Acampa and Guideri 2006).

So far, reports have shown a reduced size of the brains of Rett patients (Armstrong 2005). Moreover, neuronal size in cortex, thalamus, basal ganglia, amygdala and hippocampus has been reported to be decreased (Bauman et al. 1995). Also, studies on cerebral cortex indicated a less complex dendritic arborisation and decreased numbers of dendritic spines. Furthermore, less afferent axons were found in Rett mice as well as in patients (Belichenko et al. 1994; Belichenko and Dahlstrom 1995; Belichenko et al. 2009).

Although obvious brain structural and functional impairments in Rett syndrome are manifesting, no clear neuronal or glial cell atrophy or degeneration as well as no gliosis, demyelization or disruption of neuronal 
migration have ever been reported. Therefore, Rett syndrome is not considered as a neurodegenerative disorder (Jellinger et al. 1988; Reiss et al. 1993; Armstrong 2005).

\subsection{Genetic cause of Rett syndrome}

Mutations in the MECP2 gene, encoding the methyl-CpG binding protein 2 (MECP2), are the primary cause of Rett syndrome, and they have been confirmed in more than $95 \%$ of typical cases of this disorder (Amir et al. 1999). MECP2 is located on chromosome $X$ in the $X q 28$ band position and it consists of four exons. Due to alternative splicing of exon 2 and alternative use of the polyadenylation sites in the 3' untranslated region (3'UTR), two different variants of MECP2 transcripts can be created. It has been shown that expression of the MECP2-e1 longer transcript in brain is high especially during embryonic development, but it decreases in the postnatal period, and increases again in adulthood (Shahbazian et al. 2002; Pelka et al. 2005).

De novo mutations in MECP2 occur in the male germline, and therefore they are transmitted from the father (Trappe et al. 2001). Mutations of MECP2 involve transition of C to T at CpG dinucleotides (Wan et al. 1999; Trappe et al. 2001), and include missense, nonsense and frameshift mutations as well as entire exons' deletions (Christodoulou et al. 2003; Archer et al. 2006). Although Rett syndrome is a sporadic disorder, arising from spontaneous sperm mutations, $\sim 1 \%$ of reported cases support a genetic basis for the disease and a damaged copy of MECP2 from one inheritance of generation to the next (Zoghbi 1988; Schanen et al. 1997).

However, also atypical forms of Rett syndrome exist. They arise from mutations in the cyclin-dependent kinase-like 5 (CDKL5) gene (Tao et al. 2004; Weaving et al. 2004; Weaving et al. 2005; Archer et al. 2006). Moreover, congenital variants of Rett syndrome are caused by mutations in the forkhead box protein G1 (FOXG1) gene (Ariani et al. 2008). 


\subsection{MeCP2 protein - structure, function and expression}

MeCP2 is a $53 \mathrm{kDa}$ protein and it is a member of the methyl-CpG binding protein family (Hendrich and Bird 1998). It consists of three main domains: methyl-CpG binding domain (MBD), transcriptional repression domain (TRD) with two nuclear localization signals (NLSs), and C-terminal domain (CTD) (Figure 1).

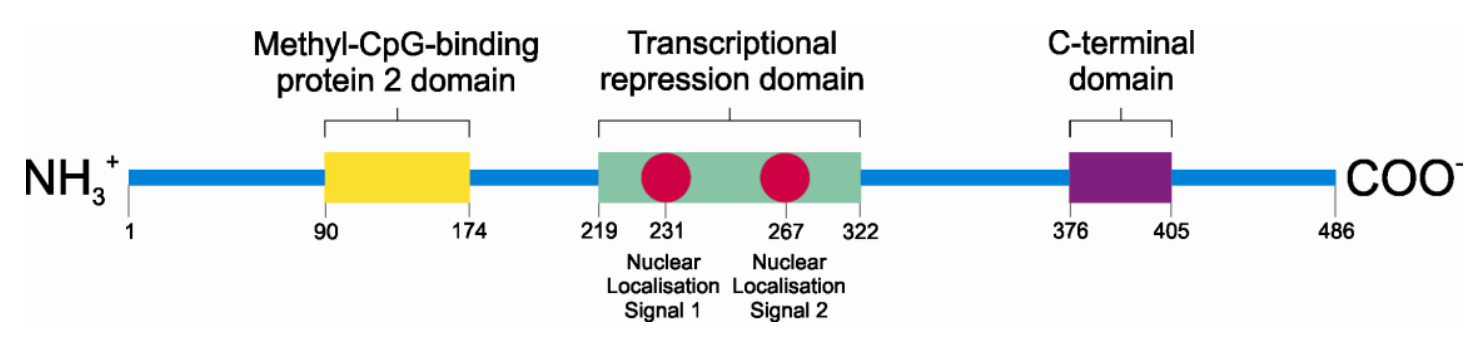

\section{Figure 1. MeCP2 structure.}

The MeCP2 protein consists of three main domains, and two of them are especially important for MeCP2 function: methyl-CpG binding domain and transcriptional repression domain, which includes two nuclear localization signals. Third domain of MeCP2 is the C-terminal domain.

The MBD domain binds specifically to methylated $\mathrm{CpG}$ dinucleotides, allowing therefore for MeCP2-chromatine interactions and chromatine modulations. However, the presence of adjacent A/T base pairs for efficient MeCP2-DNA binding is mandatory (Klose et al. 2005). Yet, an equally efficient methylationindependent form of MeCP2-DNA binding was also identified (Galvao and Thomas 2005). The TRD domain consists of residues that were originally thought to be required only for transcriptional repression (Nan et al. 1997). The hypothesis of transcriptional silencing (Figure $2 \mathrm{~A}$ ) is based on the binding of SIN3 transcription regulator family member A (Sin3A) to MeCP2 protein, which recruits histone deacetylases (HDACs) and mediates posttranscriptional modifications of histone tails (Chahrour et al. 2008).

On the other hand, MeCP2 acts also as a transcriptional activator (Figure 2 B), and by microarray analysis it was found to activate the majority ( $85 \%)$ of genes in the hypothalamus (Chahrour et al. 2008). Also, mass spectrometry analysis revealed that the CAMP responsive element binding protein 1 (CREB1) is the major co-activator, which binds to MeCP2 protein and thus, 
allows for a successful transcription of target genes (Chahrour et al. 2008). The CTD domain of MeCP2 is not yet completely known. Nevertheless, data on mice lacking the CTD domain show that CTD is necessary for proper MeCP2 function and facilitates the MeCP2-DNA binding processes (Shahbazian et al. 2002).
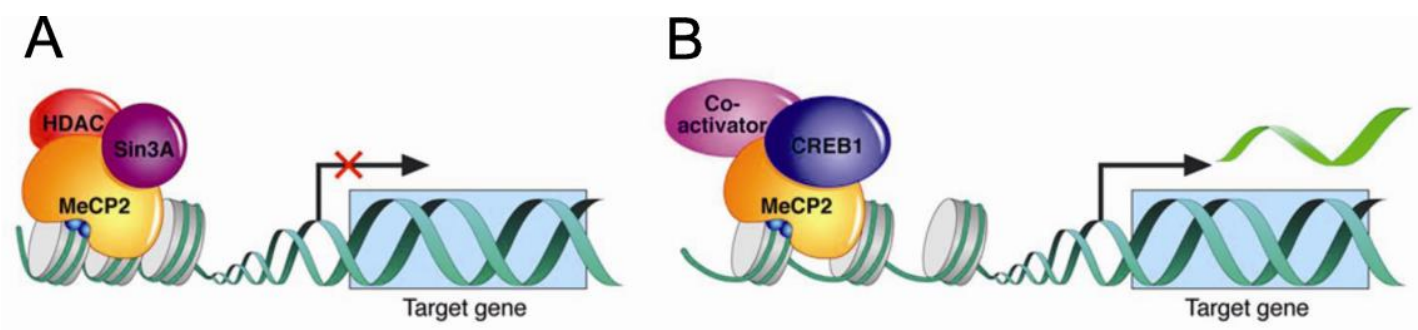

Figure 2. MeCP2 acts as a transcriptional repressor and activator.

A) The TRD domain of MeCP2 binds to the Sin3A co-repressor and recruits HDAC, causing transcriptional repression of MeCP2 target genes.

B) Due to interaction of MeCP2 with co-activators such as CREB1, successful transcription of MeCP2 target genes may take place.

From Chahrour M, Jung SY, Shaw C, Zhou X, Wong ST, Qin J, Zoghbi HY. MeCP2, a key contributor to neurological disease, activates and represses transcription. Science 2008; May 30; 320(5880):1224-9. Reprinted with permission from AAAS.

MeCP2 is a nuclear and multifunctional protein. Apart from its role in transcriptional repression and activation as well as chromatin modulation, it modulates neuronal development and neuronal differentiation. Studies on MeCP2 expression have confirmed a changing pattern of MeCP2 protein levels. MeCP2 expression is low during embryogenesis and increases during neuronal maturation at the postnatal stage, finally reaching its peak in mature neurons (Shahbazian et al. 2002; Balmer et al. 2003; Cohen et al. 2003; Kishi and Macklis 2004; Mullaney et al. 2004). Thus, it has been suggested that MeCP2 is involved in modulation of general activity and/or plasticity of mature neurons (Chahrour and Zoghbi 2007). Furthermore, MeCP2 was reported to modulate RNA splicing (Young et al. 2005). 


\subsection{Animal models of Rett syndrome}

Over the years, various mouse models of Rett syndrome have been developed and they include Mecp2 knockout, Mecp2 mutant and MECP2 knock-in mice.

The 'Bird strain' Mecp2 $2^{\text {tm1.1Bird }}$, which was used in this study, represents a Mecp2-deficient mouse model. Its Mecp2 gene lacks exons 3 and 4 and therefore, it remains completely dysfunctional with no expression (Guy et al. 2001). Mouse strain Mecp2 $2^{\text {tm1Tam }}$, which was generated by removal of the coding sequence of the MBD domain of MeCP2, constitutes another Mecp2null mouse (Pelka et al. 2006). The Mecp2-null male mice develop hindlimb clasping and reduced spontaneous movements, growth arrest, uncoordinated gait, irregular breathing and increased mortality (Guy et al. 2001). These mice die around postnatal day (PD) 50. Moreover, studies on brain sections from cerebral cortex, cerebellum and hippocampus showed smaller size and increased density of neuronal cell bodies and nuclei (Chen et al. 2001; Fischer et al. 2009). The Mecp2-null females represent only the minimal initial phenotype. They regularly take care of their offspring, live more than a year and develop clinical features only with a marked delay (Guy et al. 2001).

The group of Mecp2 mutant mouse models are mice that express only a truncated MeCP2 protein, cell-type-specific Mecp2 deletions and mutations or reduced levels of Mecp2 (Calfa et al. 2011). The MECP2 knock-in mice carry human MECP2 mutations associated with Rett syndrome and hence, they represent a cohort of experimental animal models mimicking the specific mutations of Rett syndrome in humans (Lawson-Yuen et al. 2007; Jentarra et al. 2010; Wegener et al. 2014). So far, all of the developed mouse models of Rett syndrome reveal behavioural symptoms and clinical features that are associated with the disease (Ricceri et al. 2008; Tao et al. 2009). Yet, they clearly differ in the severity of symptoms and the time course at which they appear. 
Interestingly, a novel Mecp2 knockout rat model of Rett syndrome has recently become available, and very first studies on neuronal behavioural processing of communication in Rett syndrome have been performed (Engineer et al. 2015). However, it should be mentioned that none of the various rodent models fully corresponds to the complex clinical conditions of the human disorder.

\subsection{Breathing impairment and mitochondriopathy in Rett syndrome}

It is commonly known, that Rett syndrome is associated with severe and lifethreating breathing irregularities, causing a sudden death (Kerr et al. 1997; Julu et al. 2001; Acampa and Guideri 2006). Also, the Mecp2 $2^{\text {tm1.1Bird-null }}$ mouse model (Guy et al. 2001) for Rett syndrome shows such respiratory disruptions. After PD 20 these Mecp2-deficient mice develop erratic breathing characterized by alternating fast and slow breathing frequencies as well as long-lasting apnoeas, resulting in drops of arterial oxygen saturation defined as intermittent systemic hypoxia (Julu et al. 2001; Viemari et al. 2005; Ogier and Katz 2008; Stettner et al. 2008; Katz et al. 2009).

Recent data suggest an enhanced hypoxia susceptibility of neuronal networks of hippocampus and brainstem in Mecp2-null mice. As possible underlying mechanisms, contributing to this increased hypoxia susceptibility, disruption of $\mathrm{K}^{+}$channel function as well as an imbalance in $\mathrm{Ca}^{2+}$ homeostasis were proposed (Fischer et al. 2009; Mironov et al. 2009; Kron and Müller 2010; Kron et al. 2011). Also, since mitochondria are actively involved in the $\mathrm{Ca}^{2+}$ uptake and $\mathrm{Ca}^{2+}$ release pathways (Duchen 2000; Brookes et al. 2004), it is tempting to take a closer look at these organelles.

Indeed, various signs of mitochondrial pathology have been found in tissue samples from Rett patients and mouse models. A case report on the frontal lobe biopsy tissue of a Rett individual at age 3 showed impaired mitochondrial morphology, which became evident due to numerous large and spherical mitochondria, low-density structure of mitochondrial matrix, few rudimentary 
cristae and abundant ribosomal content. Moreover, electron microscopy studies on brain post-mortem tissue from the same 15 years old patient showed granular matrix inclusions and disorganized membranous material (Cornford et al. 1994). Basing on muscle biopsy material, also mitochondrial distention, vacuolisation as well as irregular cristae were uncovered by other studies (Ruch et al. 1989; Eeg-Olofsson et al. 1990).

Apart from morphological studies, strong evidence of mitochondrial enzyme impairment has been also reported (Coker and Melnyk 1991; Gibson et al. 2010). Enzyme assays performed on muscle biopsy material revealed lower levels of cytochrome $c$ oxidase and succinate cytochrome $c$ reductase in three Rett girls (Coker and Melnyk 1991). Furthermore, the cytochrome $c$ oxidase subunit 1 was downregulated in the frontal cortex of Mecp2-deficient mice (Gibson et al. 2010).

Mitochondrial impairment has been confirmed also on the gene level. Gene expression profiles, which were performed on the peripheral blood lymphomonocytes from Rett patients by using microarray assays, suggest misregulation of 482 genes, out of which 146 genes are involved in mitochondrial function, ubiquitination, proteosome degradation, mitochondrial organisation, ATP-synthesis, chromatin modulation and RNA processing (Pecorelli et al. 2013). Moreover, expression analyses performed on Rett mice identified an overexpression of the ubiquinol-cytochrome $c$ reductase core protein 1 (Uqcrc1), which encodes for a complex III subunit of the mitochondrial respiratory chain, and whose promoter interacts with MeCP2 (Kriaucionis et al. 2006). Along with these findings, a potential uncoupling and $30 \%$ more intensified respiration rates of isolated brain mitochondria were identified. However, due to various tissue processing and mRNA extraction protocols, microarray studies might lead to differing results and quite variable gene expression patterns. Moreover, it should be mentioned, that a change on the gene level does not always correspond to a downstream modulation of protein activity (Li et al. 2013). 
Evidence for altered mitochondrial function as well as oxidative changes has been also reported by our group. An increased FAD/NADH autofluorescence ratio in both neonatal and adult Rett mice was detected, confirming more intensively respiring mitochondria (Großer et al. 2012; Müller and Can 2014). Based on blood analyses on human patients, a reduced activity of the scavenging enzyme SOD (Sierra et al. 2001) and decreased levels of vitamin E were identified, which also plays an important role in the cellular scavenging system (Formichi et al. 1998).

Other evidence for a functional impairment of mitochondria in Rett syndrome includes variations in mitochondrial ATP production and energy disturbances. Indeed, Saywell and colleagues showed decreased ATP levels in Rett mice, which was detected by using magnetic resonance imaging (Saywell et al. 2006). Our group could, however, not confirm these observations as no significant changes in ATP levels between adult hippocampus of Mecp2-null and the respective WT tissue have been observed (Fischer et al. 2009). Interestingly, increased cellular ATP levels and more intensified ATP turnover have been recently reported for neonatal Mecp2-deficient hippocampus by using the ATP-specific genetically-encoded sensor Ateam 1.03 (Toloe et al. 2014).

\subsection{Reactive oxygen species}

Mitochondria are considered to be one of the most important and major generators of superoxide $\left(\cdot \mathrm{O}_{2}^{-}\right)$(Loschen et al. 1971; Boveris et al. 1972). Approximately $2-5 \%$ of electrons escape during the electron transit within the respiratory chain from complex I to complex IV and bind to molecular oxygen, forming thereby $\mathrm{O}_{2}^{-}$and contributing to the secondary production of other reactive oxygen species (ROS) such as hydroxyl radicals $(\cdot \mathrm{OH})$ at complexes I and II (Boveris and Chance 1973).

Superoxide anions are highly unstable and reactive molecules. They rapidly convert into the somewhat less reactive $\mathrm{H}_{2} \mathrm{O}_{2}$. The $\mathrm{H}_{2} \mathrm{O}_{2}$ might form $\cdot \mathrm{OH}$ and hydroxyl anions $\left(\mathrm{OH}^{-}\right)$in the presence of iron $\left(\mathrm{Fe}^{2+}\right)$ due to electron 
acceptance from a transition metal in a Fenton reaction. Furthermore, it can generate peroxynitrite ( $\mathrm{ONOO}^{-}$) in a reaction with nitric oxide (NO) (Dean et al. 1997; Lipton and Nicotera 1998) (Figure 3).

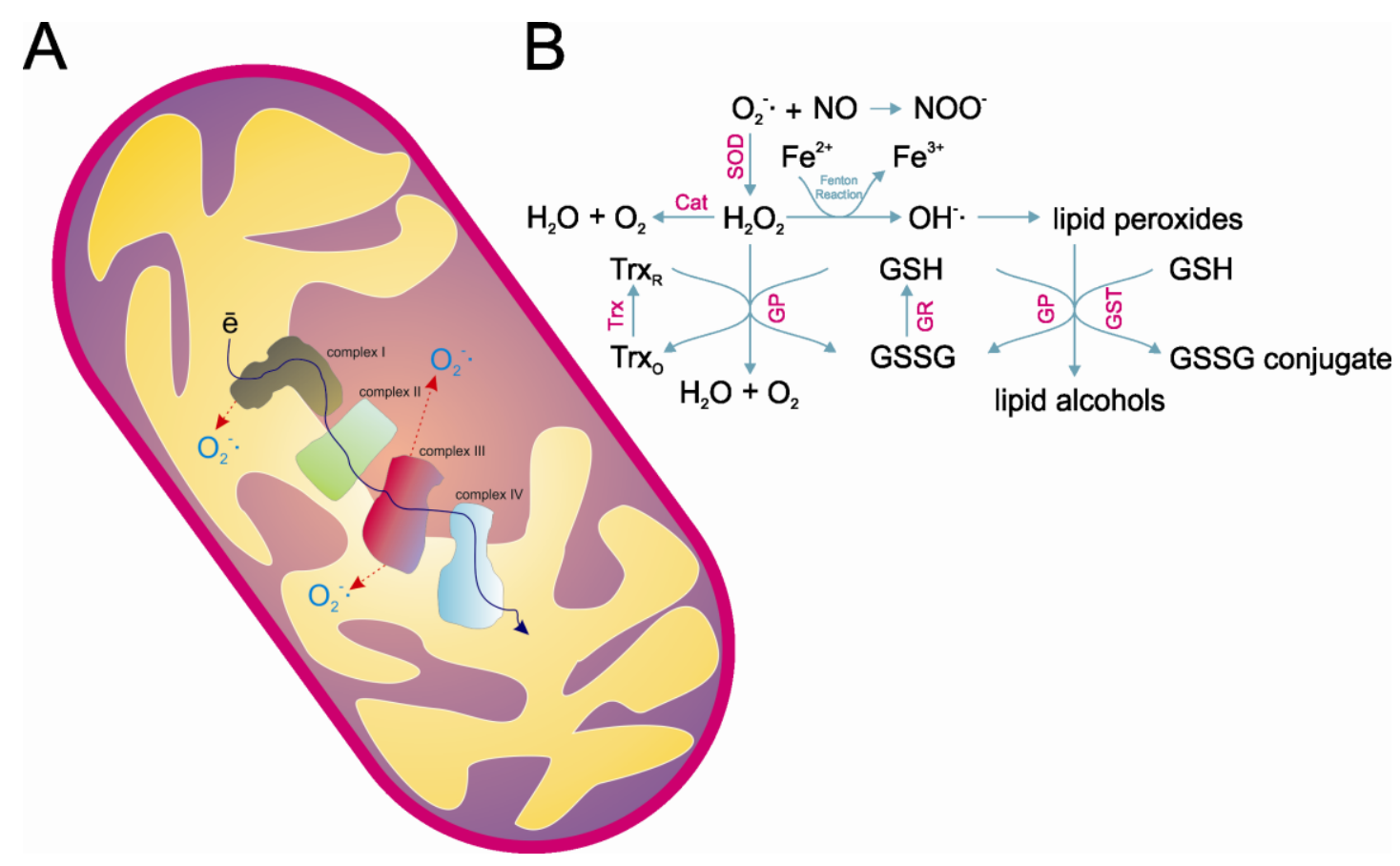

Figure 3. ROS generation and cellular scavenging systems

A) The respiratory chain complexes I and III generate $\cdot \mathrm{O}_{2}^{-}$due to the electron (ē) flow in mitochondria.

B) The superoxide dismutase (SOD); catalase (Cat), thioredoxin reductase (Trx); glutathione peroxidase (GP), glutathione reductase (GR) and glutathione $S$-transferase (GST) constitute the main components of the cellular anti-oxidant defence. GSH represents monomeric glutathione, and GSSG represents the oxidized glutathione disulfide. NO, nitric oxide; ONOO, peroxynitrite.

Generation of ROS within mitochondria is constant and low levels of ROS play an important role in cellular physiology. ROS were proven to participate in various cellular signalling pathways, e.g. the control of the vascular tone in the brain (Demchenko et al. 2002). Furthermore, they take part in host defence and cellular proliferation, and they are also involved in aging and cell apoptosis processes (Sinha et al.; Harman 2003; Kroncke 2003; Nathan 2003; Finkel 2011; Sinha et al. 2013). 
However, any imbalance in ROS production due to mitochondrial dysfunction, e.g. enhanced mitochondrial activity, respiratory chain inhibition or disruption in the cellular ROS neutralization systems, leads to oxidative stress, and may therefore seriously threaten cell viability or cell function (Sies 1991; Halliwell and Cross 1994). Due to oxidation challenge, highly activated ROS constitute stress signals, causing DNA damage, protein nitrosylation, lipid peroxidation and alterations in DNA methylation level, and finally changes in gene expression (Weitzman et al. 1994) (Figure 4). Yet, the severity of cellular changes due to ROS interference depends on the exact type of ROS. Whereas $\mathrm{H}_{2} \mathrm{O}_{2}$ seems to be less harmful, $\mathrm{O}_{2} \cdot{ }^{-}$, $\cdot \mathrm{OH}$ and $\mathrm{ONOO}^{-}$are considered to be very aggressive in their reactivity (Halliwell and Gutteridge 1984; Lipton 1999; Chan 2001; Foster et al. 2006).

To prevent intensified ROS accumulation, cells developed various enzymatic defence systems, e.g. catalase (Cat), superoxide dismutases (SOD1, SOD2, SOD3), glutathione peroxidase (GP), glutathione $S$-transferases (GST), glutathione reductase (GR), peroxiredoxins (Prxs), glutaredoxin and two thioredoxin isoforms (TRX1 and TRX2). Also, non-enzymatic scavengers take part in the cell endogenous protection against ROS, e.g. glutathione disulfide (GSH) thioredoxin, $\alpha$-tocopherol (vitamin E), ascorbic acid (vitamin C), beta carotene, uric acid and melatonin (Sinha et al.; Milton and Sweeney 2011; Sinha et al. 2013) (Figure 3). 
A
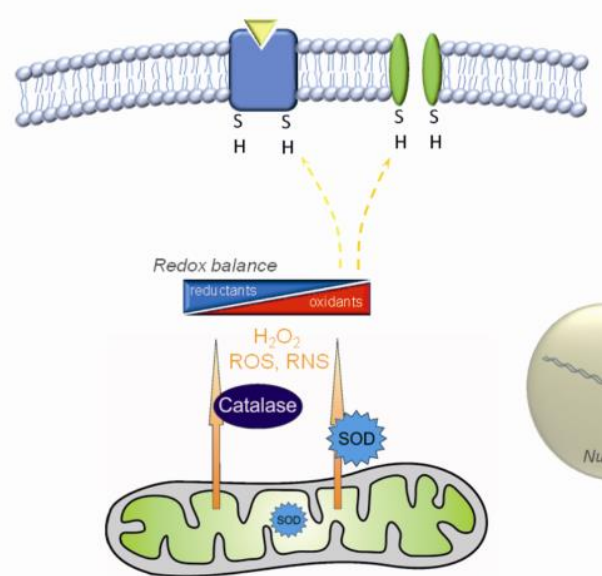

B

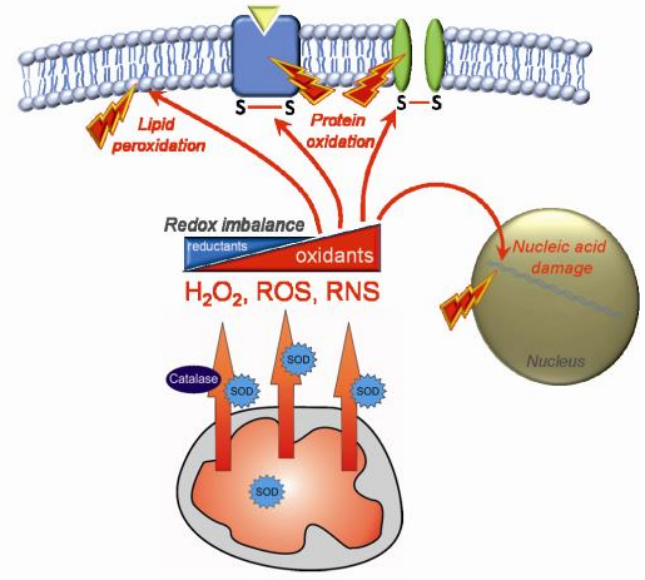

Figure 4. ROS as a potential source of cellular damage

A) Healthy mitochondria generate only low levels of ROS and downstream reactive nitrogen species (RNS). Redox balance is guaranteed due to properly functioning cellular scavenging systems.

B) Impairment of mitochondria or less efficient scavenging capabilities may, however, give rise to abnormally intensified generation of ROS. This unequivocally culminates in aberrant redox conditions and oxidative stress, and therefore, it may cause profound cellular damage. This research was originally published in Portland Press Limited. Müller M, Can K. Aberrant redox homoeostasis and mitochondrial dysfunction in Rett syndrome. 2014 Aug; 42(4):95964, Biochemical Society Transactions.

\subsection{Oxidative stress in Rett syndrome and oxidative stress markers}

Cellular alterations elicited by ROS have been confirmed in various neurodevelopmental and neurodegenerative disorders (Fernández-Checa et al. 2010). For example, oxidative damage was proven to play a substantial role in the development of Alzheimer's disease, Parkinson's disease, Huntington's disease or Amyotrophic lateral sclerosis (Zhang et al. 1999; Rahman et al. 2012). Oxidative stress can provoke neuronal cell dysfunction and/or neuronal death and therefore, it might drive disease progression. Hence, studies on the detailed effects mediated by ROS constitute a central focus of research over the last years.

Also for Rett syndrome, extended studies have confirmed an involvement of oxidative stress. Already in 1987, reduced levels of ascorbic acid and 
glutathione were found in post-mortem brain tissue of Rett patients (Sofić et al. 1987). Furthermore, lipid peroxidation and significantly elevated plasma protein carbonyl levels as well as increased hypoxia-induced oxidative stress were demonstrated in patients with typical Rett syndrome (De Felice et al. 2009). Furthermore, studies on Rett patients clearly indicated mutations in target genes of MeCP2 such as the brain-derived neurotrophic factor (BDNF) and CREB (De Felice et al. 2009). These genes were proven to be involved not only in neuronal protection against hypoxia, but also in ROS scavenging (Lee et al. 2009; Pereira et al. 2009). In addition, recent data pointed out an important role of the insulin-like growth factor 1 (IGF-1). It mediates neuroprotection against oxidative stress under control conditions (Heck et al. 1999), and it has been also shown to partially reverse some of the Rett syndrome-like symptoms by e.g. improved spine density, synaptic potential restoration and cortical plasticity stabilization (Tropea et al. 2009). Moreover, further evidence of a direct connection between MeCP2 and oxidative stress was reported by the group of Valinluck as the affinity properties of the MBD domain of MeCP2 were found to be inhibited by oxidative damage (Valinluck et al. 2004). Accordingly, such ROS-mediated changes might therefore culminate in epigenetic DNA damage and disrupt chromatin organization.

Further investigations of parameters, which might be involved in functional neuronal disruptions in Rett syndrome, revealed changes in the antioxidant defence responses. For example, SOD activity was found to be significantly decreased in erythrocytes of Rett patients (Sierra et al. 2001). Following these studies, other data showed critically increased levels of malondialdehyde - a marker for content lipid peroxidation - also in Rett individuals. This could possibly lead to peroxidative damage of cellular membranes, and finally in consequence to progression of dementia, impaired motor function, changes in behaviour and seizure incidence (Ross 2000; Sudha et al. 2001). Nevertheless, there are numerous and currently available oxidative stress markers that are specific for pro-oxidant factors, lipid peroxidation and protein oxidation as well as for mitochondrial impairment, enzyme activity and gene expression (De Felice et al. 2012). Among this large group of biomarkers, isoprostanes (IsoPs) - the prostaglandin-like 
compounds - represent a reliable class of bioproducts of oxidative imbalance (Kadiiska et al. 2005). They originate from the peroxidation of polyunsaturated fatty acid precursors and fulfil a diversity of biological functions. For example, they take part in inflammatory responses and act as pain mediators or neuromodulators (Jahn et al. 2008; Milne et al. 2011). Additionally, reduced vitamin E concentrations were identified in Rett patients' serum, suggesting less efficient antioxidant capacity in Rett syndrome (Formichi et al. 1998).

\subsection{Imaging of reactive oxygen species - outline of redox sensors}

Over the years, various dyes and ROS/redox sensors for imaging of ROS formation and/or oxidative stress detection became available. Compounds such as dichlorodihydrofluorescein (LeBel et al. 1992) dihydroethidium (Gallop et al. 1984) and Amplex Red (Mohanty et al. 1997) accumulate in the cytosol after entering a cell and become fluorescent once being oxidized due to cellular ROS production.

To evaluate and detect ROS/redox changes directly in mitochondria, other synthetic dyes targeting the mitochondrial matrix were generated, namely dihydrorhodamine (Dugan et al. 1995), RedoxSensor Red CC-1 Stain (Chen and Gee 2000), MitoSOXRed (Robinson et al. 2006), and the MitoTracker probes, e.g. MitoTracker Red $\mathrm{CM}-\mathrm{H}_{2} \mathrm{XROS}$, MitoTracker Orange CM$\mathrm{H}_{2}$ TMROS (Esposti et al. 1999). Due to very intense fluorescence of these synthetic dyes, mitochondrial morphology as well as tracking of mitochondrial particles and mitochondrial content might be also assessed within a single cell (de la Monte et al. 2000; Stamer et al. 2002). All of these redox indicators become intensively fluorescent only upon oxidation. But this conversion is irreversible. Moreover, any sensitivity of these dyes to reducing conditions is lacking. Thus, quantifiable evaluation of cellular ROS levels and/or redox changes is not achievable. Although these ROS dyes are relatively easy to handle and sufficient cell loading occurs within minutes, other disadvantages such as fast photo-bleaching, auto-oxidation, and/or accumulation in other 
compartments restrict a reliable functional analysis of ROS levels and their changes within a cell.

Yet, the genetically-encoded optical redox indicators are more reliable as they react to both oxidation and reduction, allowing therefore for a quantitative assessment of cellular ROS/redox changes. Moreover, they overcome most of the problems associated with photobleaching and cell movements, and they can be designed to target a specific type of cells, defined cellular compartments or even particular parts of protein (Gutscher et al. 2008). These optical sensors derive from the green and yellow fluorescent proteins (YFP, GFP). Redox-sensitive properties of these indicators are achieved by specific structural modification of the native YFP and GFP - redox-sensitive yellow fluorescent protein (rxYFP) and reduction/oxidation-sensitive green fluorescent protein (roGFP) (Ostergaard et al. 2001; Hanson et al. 2004). Mainly, it is the insertion of reactive cysteines within the fluorescent proteins, which generate a functional switch between the oxidized and reduced forms of the protein. The fluorescent hydroxyl peroxide (HyPer) sensor (Belousov et al. 2006) together with the fluorescence resonance energy transfer (FRET) constructs such as CFP-HSP33-YFP (Guzy et al. 2005), CFP-RL5-YFP (Kolossov et al. 2008) and Redoxfluor (Cerulean -Yap1-Citrine)(Yano et al. 2010) are also members of the group of genetically-encoded optical probes. Nevertheless, especially the optical sensor roGFP1 is used for quantitative and dynamic redox imaging by our group. Since roGFP1 responds reversibly to oxidation/reduction due to the functional thiol switch, and reacts similarly to other redox-sensitive proteins, it may give detailed information on the other redox-modulated endogenous proteins. So far, our group confirmed reliable responses of roGFP1 in both murine and rat hippocampal neurons in a variety of oxidizing and reducing conditions. Moreover, in terms of response reliability, data from our laboratory ruled out any significant effects of intracellular $\mathrm{pH}$ or $\mathrm{Cl}^{-}$changes on roGFP1 (Funke et al. 2011; Großer et al. 2012). Further details about roGFP1 and HyPer sensors are given in the following Materials and Methods section. 


\section{Aims of this Thesis}

Although the literature is constantly growing with new findings on mitochondrial impairment, those mechanisms leading to MeCP2 dysfunction and Rett syndrome progression are still lacking detailed explanation. A variety of open questions, regarding a direct connection between oxidative stress and Rett syndrome as well as the molecular causes of oxidative imbalance, and the very reasons for damaged mitochondria still need to be answered.

The aim of this thesis was to analyze redox changes in $M e c p 2^{-/ y}$ mice specifically in primary cultured hippocampal neurons as well as in organotypic hippocampal slices. The hippocampal formation is an established model for metabolic insults, neurodegeneration, mitochondrial and cellular signalling dysfunction, and it has been proven to respond more sensitively than other tissues to oxidative challenge (Wilde et al. 1997; Wang et al. 2007). Since the mitochondrial matrix constitutes the primary cellular source of ROS, and since ROS might be further released from mitochondria into the cytosol within a cell (Brand 2010), evaluation of redox changes in both mitochondrial and cytosolic compartments was considered to be mandatory.

Therefore, the main objectives of the current thesis were:

1. First of all, in order to analyse sub-cellular redox changes within neurons, a suitable and reliable redox indicator had to be chosen.

2. To investigate mitochondrial and cellular redox changes specifically in hippocampal neurons, viral vectors carrying the respective cytosolic and mitochondrial optical redox-sensor roGFP1 were established to optimize expression levels and the selectivity of redox sensor expression.

3. Detailed calibration of the selected redox sensor roGFP1 was performed to enable quantitative recordings of redox balance. 
4. Redox responses were assessed upon control and stress conditions, e.g. hypoxia, oxidative challenge, blockade of the scavenging enzyme SOD, mitochondrial respiration inhibition and application of various neurotransmitters to define altered responses in $M e c p 2^{-/ y}$ neurons.

5. To analyse further sub-cellular changes of Mecp2 $2^{-/ y}$ and WT neurons, local neuronal stimulation was performed, using both pressure drug ejection and extracellular electrical stimulation.

6. To decipher potential candidate processes, underlying the detected redox alterations in $M e c p 2^{-/ y}$, contribution of signalling pathways such as $\mathrm{Ca}^{2+}$ influx, role of mitochondria, involvement of NADPH oxidase and xanthine oxidase as well as an activation of G-proteins were analyzed.

7. To extend quantitative redox imaging to adult $M e c p 2^{-/ y}$ mice, the crossbreeding of the recently generated transgenic roGFP1c mice with Mecp2 $^{+/ y}$ mice has been started to be able to correlate redox imbalance with disease progression of Rett syndrome. 


\section{Materials and Methods}

All experiments were conducted in accordance to German national regulations. The tissue isolation and the in vitro experiments were approved by the office for animal welfare of the Universitätsmedizin Göttingen "Anoxie und neuronale Netzwerke (T13-08)".

\subsection{Solutions and pharmacological compounds}

Usually, chemicals obtained from Sigma-Aldrich were used, in other cases this is clearly stated.

To maintain viability of primary hippocampal cell and organotypic slice culture during particular experiments, the artificial cerebrospinal fluid (ACSF) was used. It was composed of (in mM) $130 \mathrm{NaCl}, 3.5 \mathrm{KCl}, 1.25 \mathrm{NaH}_{2} \mathrm{PO}_{4}, 24$ $\mathrm{NaHCO}_{3}, 1.2 \mathrm{CaCl}_{2}, 1.2 \mathrm{MgSO}_{4}$ and 10 dextrose. During the entire experiment, the ASCF was constantly aerated with carbogen $\left(95 \% \mathrm{O}_{2}, 5 \%\right.$ $\mathrm{CO}_{2}$ ) in order to guarantee the constant oxygenation of cell and slice cultures, and to preserve a stable $\mathrm{pH}$ of 7.4 .

In order to challenge cell and slice cultures by various oxidizing and reducing conditions, different drugs were administered. These include: $\mathrm{H}_{2} \mathrm{O}_{2}(30 \%$ stock solution in $\mathrm{H}_{2} \mathrm{O}$ ), diethyldithiocarbamic acid (DEDTC), 1,4-dithio-DLthreitol, (DTT, Fluka), antimycin A (AMC, $20 \mathrm{mM}$ stock solution in ethanol, stored at $-20^{\circ} \mathrm{C}$ ), dimethyl sulfoxide (DMSO), propionate, tertbutylhydroperoxide (tBHP, $70 \%$ stock solution in $\mathrm{H}_{2} \mathrm{O}$ ), forskolin $(20 \mathrm{mM}$ stock solution in DMSO, stored at $-20^{\circ} \mathrm{C}$, Bio Trend), carbonyl cyanide4(trifluoromethoxy)phenylhydrazone (FCCP, $10 \mathrm{mM}$ stock solution in DMSO, stored at $4^{\circ} \mathrm{C}$, Tocris Bioscience), diphenyleneiodonium chloride (DPI, $10 \mathrm{mM}$ stock solution in DMSO, stored at $-20^{\circ} \mathrm{C}$, Tocris Bioscence) and cyanide $\left(\mathrm{CN}^{-}\right.$, $1 \mathrm{M}$ stock solution in $\mathrm{H}_{2} \mathrm{O}$, stored at $-20^{\circ} \mathrm{C}$ ). Glutamate ( $5 \mathrm{mM}$ stock solution in $\mathrm{H}_{2} \mathrm{O}$, stored at $-20^{\circ} \mathrm{C}$ ), norepinephrine, serotonin, dopamine and allopurinol were immediately dissolved in ACSF in their final concentrations right before use. 
To calibrate the $\mathrm{pH}$-sensitive optical sensor SypHer, a calibration solution was prepared from (in $\mathrm{mM}$ ) $130 \mathrm{KCl}, 1.2 \mathrm{CaCl}_{2}, 1.2 \mathrm{MgCl}_{2}, 10$ glucose, 20 4-(2hydroxyethyl)piperazine-1-ethanesulfonic acid (HEPES) and adjusted up to $\mathrm{pH} 7.0$ by $\mathrm{NaOH}$, in order to mimic intracellular ionic conditions. The final calibration solutions were adjusted with $\mathrm{KOH}$ to the respective $\mathrm{pH}: 7.0,7.2$, 7.4, 7.6, 7.8. To disrupt the membrane potential in neurons, $5 \mu \mathrm{M}$ nigericin (2.5 mM stock solution in DMSO and 100\% ethanol in proportion 1:1, stored at $-20^{\circ} \mathrm{C}$ ) was used.

\subsection{Mouse model of Rett syndrome}

The Mecp2-deficient mouse model represented by the 'Bird strain' Mecp2 $2^{\text {tm1.1Bird }}$ was used for the current work (Guy et al. 2001). The heterozygous $\left(\mathrm{Mecp}^{+/}\right)$female mice were provided by Jackson Laboratories (Bar Harbor, Me.) and next bred with C57BL/6J WT males (Figure 5). Mice were maintained in the central animal facility of the University of Göttingen in a stable $12 \mathrm{~h}$ light-dark cycle, and they had a constant access to food and water. In order to genotype WT and Mecp2-/y mice, the polymerase chain reaction (PCR) was performed on a tail biopsy material. For all hippocampal cell and slice culture preparations, Mecp2 $2^{-/ y}$ mice at PD 2-4 were used.

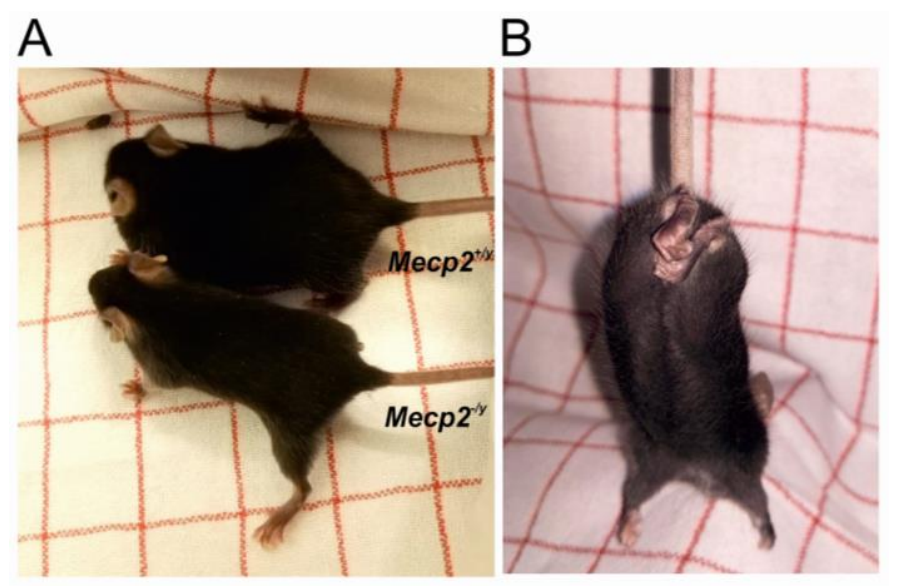

Figure 5. The 'Bird strain' Mecp2 ${ }^{\text {tm1.1Bird }}$ mouse model of Rett syndrome.

A) During development, $M e c p 2^{-/ y}$ mice start developing a very obvious phenotype from $\sim$ PD 20 and this includes markedly reduced gain of body weight, lower body temperature, loss of mobility and irregular breathing.

B) Most of symptomatic Mecp2 $2^{-y}$ mice show also an obvious hind-limb clasping. 


\subsection{Preparation of dissociated cell and organotypic cultures}

Preparation of primary hippocampal dissociated neuronal cultures was performed according to the previous protocol from our group (Weller et al. 2014). Neonatal Mecp2-y males at PD 1-4 were decapitated and the whole brain was isolated from the skull into ice-cold Hanks' balanced salt solution (HBSS), containing 20\% FCS (fetal calf serum, Biochrom). Hippocampi were then dissected, cleaned from additional cortical tissue and the meninges, and next cut into several pieces. After two washing steps with HBSS, containing $20 \%$ FCS, and three further washing steps with HBSS without serum, hippocampi were treated with freshly prepared digestion solution for $10 \mathrm{~min}$ at $37^{\circ} \mathrm{C}$ in a humidified $\left(5 \% \mathrm{CO}_{2}\right)$ incubator. After trypsinization, hippocampi were washed again with HBSS with 20\% FCS and HBSS only. They were triturated in a freshly prepared dissociation solution, and the neuronal cell suspension obtained was then centrifuged (1500 rpm, $10 \mathrm{~min}, 4^{\circ} \mathrm{C}$ ). The cell pellet was redissolved in ice-cold plating medium and neurons were plated on sterile glass coverslips, coated beforehand with Matrigel (BD Biosciences). Final culturing was done in four-well plates (Nunc). The cell density of the plated neurons was at about 30.000-40.000 cells per well. At first, the dissociated primary neurons were incubated at humidified conditions $\left(37^{\circ} \mathrm{C}\right.$, $5 \% \mathrm{CO}_{2}$ ) for a minimum of $24 \mathrm{~h}$. After one day, plating medium was replaced by growing medium. In the following culturing period, $50 \%$ of medium was replaced by pre-warmed and fresh growing medium every 2-3 days.

To prepare organotypic slice culture, earlier protocols from our group were used (Fischer et al. 2009; Kron and Müller 2010; Großer et al. 2012). The $M_{\text {Mecp2 }}{ }^{-1 y}$ and WT mice at PD 2-5 were decapitated and the whole brain was isolated directly into ice-cold HBSS without serum. Hippocampi were dissected, gently cleaned from the meninges without disturbing the tissue, and next placed on a Mcllwain tissue chopper (Stoelting Co.) while protecting the tissue from drying out too much. Hippocampi were completely chopped into $350 \mu \mathrm{m}$-thick slices, which were gently separated in HBSS and transferred onto the porous support membranes of 6-well culture plates (Transwell Permeable Support, Corning). Excess of HBSS medium was 
removed using a pipette without disturbing the membranes. A maximum of 4 slices were cultured in each single well. Slices were incubated in sterile and humidified conditions $\left(37^{\circ} \mathrm{C}, 5 \% \mathrm{CO}_{2}\right)$. To maintain organotypic slice cultures, over a longer period half of the growing medium was replaced every 2-3 days.

\subsection{Culturing media}

The HBSS medium, used during tissue dissection, contained Hanks powder, $\mathrm{NaHCO}_{3} 0.35 \mathrm{~g} / \mathrm{l}$, HEPES $0.238 \mathrm{~g} / \mathrm{l}$ and $\mathrm{H}_{2} \mathrm{O}$. The $\mathrm{pH}$ was adjusted to 7.3-7.4.

To trypsinize dissected hippocampi, the digestion solution was used. It contained (in mM) NaCl 137, $\mathrm{KCl} 5, \mathrm{Na}_{2} \mathrm{HPO}_{4}$ 7, and HEPES 25. Before use, digestion solution was additionally supplemented with trypsin $0.5 \mathrm{~g} / 100 \mathrm{ml}$ and DNAse $0.05 \mathrm{~g} / 100 \mathrm{ml}$, and filtered. The $\mathrm{pH}$ was adjusted to 7.2.

Dissociation solution was required during the triturating procedure of hippocampal tissue fragments in order to obtain a cell suspension. Dissociation solution consisted of Hanks and $12 \mathrm{mM}$ of $\mathrm{MgSO}_{4}$ was enriched with DNAse $0.05 \mathrm{~g} / 100 \mathrm{ml}$, and filtered before use.

Plating medium was served for the initial plating of neuronal cell cultures, and it consisted of minimum essential medium (MEM) (Invitrogen) plus 10\% FCS, $5 \mathrm{mg} / \mathrm{ml}$ glucose, $0.2 \mathrm{mg} / \mathrm{ml} \mathrm{NaHCO} 3,0.1 \mathrm{mg} / \mathrm{ml}$ transferrin (Calbiochem), 2 $\mathrm{mM} \mathrm{L-glutamine}$ and $25 \mu \mathrm{g} / \mathrm{ml}$ insulin.

Growing medium was used for both cell and slice cultures and it contained MEM, $5 \mathrm{mg} / \mathrm{ml}$ glucose, $0.2 \mathrm{mg} / \mathrm{ml} \mathrm{NaHCO}$, $0.1 \mathrm{mg} / \mathrm{ml}$ transferrin, $5 \% \mathrm{FCS}$, $0.5 \mathrm{mM} \mathrm{L}$-glutamine, $20 \mu \mathrm{l} / \mathrm{ml}$ B27 50x supplement including antioxidants (Invitrogen), $2 \mu \mathrm{M}$ cytosine arabinoside and $100 \mu \mathrm{g} / \mathrm{ml}$ penicillin-streptomycin (Biochrom).

All culturing media were filtered and stored sterile at $4^{\circ} \mathrm{C}$. Experiments were performed between 7-16 days in vitro (DIV). 


\subsection{Preparation of brain acute slices}

To prepare acute brain slices, earlier protocols from our group were followed (Fischer et al. 2009; Großer et al. 2012). Transgenic mice, carrying cytosolic roGFP1 sensor specifically in neurons (roGFP1c) (Wagener et al. 2016) at PD 47-52, were anesthetized by using di-ethyl ether and then decapitated. The brain was isolated and placed in ice-cold ACSF for $\sim 2 \mathrm{~min}$. After a sufficient chilling period of a few min, the brain was sliced in $400 \mu \mathrm{m}$ thick coronal slices by a vibroslicer (752 M Vibroslice, Campden Instruments, Loughborough, Leicester, UK). The slices were then gently placed in a well oxygenated submersion-style chamber. They recovered at room temperature (RT) for a minimum duration of at least $90 \mathrm{~min}$. Afterwards, slices were transferred into the recording chamber, which was constantly supplied (flow rate $3-4 \mathrm{ml} / \mathrm{min})$ with aerated $\left(95 \% \mathrm{O}_{2} / 5 \% \mathrm{CO}_{2}\right)$ and pre-warmed $\left(37^{\circ} \mathrm{C}\right)$ ACSF.

\subsection{Transduction}

Primary hippocampal cell and organotypic slice cultures were transduced with the genetically-engineered optical sensor roGFP1. The roGFP1 expressing plasmid and its sequences were obtained from the University of Oregon under the permission of the inventor Prof. $S$. James Remington. The respective adenoviral constructs (AAV-6-syn roGFP1 virus), selectively targeting mitochondria (mito-roGFP1) and cytosol (cyto-roGFP1) in neurons, have been provided by Dr. Sebastian Kügler (viral vector platform, CNMPB, University Medical Center Göttingen). The human synapsin-1 promoter guaranteed a specific neuronal expression of the cyto-roGFP1 and mitoroGFP1.

Dissociated hippocampal neurons were transduced with cyto-roGFP1 or mitoroGFP1 viral vectors (stock dilution 1:50) in phosphate buffered saline (PBS) on DIV 2, respectively. To transduce neuronal cultures, $2.5 \mu \mathrm{l}$ of diluted constructs were directly added into $800 \mu \mathrm{l}$ of growing medium. 
Organotypic hippocampal slice cultures were transduced on DIV 3-4. The 2.5 $\mu \mathrm{l}$ of the cyto-roGFP1 or mito-roGFP1 (stock dilution 1:50) construct were directly applied onto the surface of each slice. Primary cell and slice cultures were left undisturbed for another 5-7 days until a sufficient roGFP1 expression ( $15 \%)$ of neurons was obtained.

\subsection{Transfection}

In order to assess, whether the genetically-encoded hydroxyl peroxide sensor HyPer is $\mathrm{pH}$ susceptible, primary neuronal cultures were transfected with the $\mathrm{pH}$-sensitive, but redox-insensitive variant of the original HyPer sensor (SypHer, pC1-HyPer-C199S, Addgene Plasmid 42213) (Poburko et al. 2011). This sensor was expressed in the cytosol of neurons and glial cells.

On DIV 2, primary neuronal cultures were transfected with the SypHer vector plasmid by using Lipofectamine 2000 (Invitrogen). In detail, plating medium (see section 3.4.) was removed and $200 \mu \mathrm{l}$ of transfection solution was added into each well. Transfection solution (OptiMEM, Invitrogen) was complemented with $1 \%$ Lipofectamine and $1 \mu \mathrm{g} / \mathrm{ml}$ DNA of the SypHer vector. Cells were placed for $1 \mathrm{~h}$ at $37^{\circ} \mathrm{C}$ in a humidified and $5 \% \mathrm{CO}_{2}$-containing incubator. Transfection solution was next replaced by fresh and pre-warmed $\left(37^{\circ} \mathrm{C}\right)$ growing medium (see section 3.4.). A sufficient fraction of SypHerexpressing primary cells (2-3\%) was obtained within $48 \mathrm{~h}$.

\subsection{Visualizing of mitochondria in vitro using MitoTracker RED FM}

To identify mitochondrial compartments within neurons, the mitochondriaspecific synthetic fluorescent dye MitoTracker RED FM (Life Technologies) was used. To visualize intact mitochondria in vitro, growing medium was removed and pre-warmed $\left(37^{\circ} \mathrm{C}\right)$ ACSF containing MitoTracker RED FM (1 $\mu \mathrm{M})$ was added to primary neuronal cultures. Cells were incubated for $20 \mathrm{~min}$ $\left(37^{\circ} \mathrm{C}, 5 \% \mathrm{CO}_{2}\right)$. Neuronal mitochondria were imaged using a 2-photon microscope equipped with a $63 \times 1.0 N A$ objective (Zeiss Plan-Apochromat VIS-IR) and a wavelength of $860 \mathrm{~nm}$. 


\subsection{Immunostaining of neurons with MAP2}

To confirm the neuronal specificity of roGFP1, primary hippocampal cultures were stained with the neuronal marker anti-microtubule associated protein 2 (MAP2) antibody (Cell Signaling).

Cultured neurons were gently washed two times with a pre-warmed PBS $\left(37^{\circ} \mathrm{C}\right.$ ) for $3 \mathrm{~min}$ and fixed in $4 \%$ paraformaldehyde (PFA) for $20 \mathrm{~min}$ at RT. Cells were washed again two more times in PBS in order to remove any remaining PFA. To permabilize cellular membranes, cells were washed two times with $0.2 \%$ triton in PBS for 3 min. Neurons were then incubated with MAP2 antibody (stock dilution 1:300) overnight (o/n) at $4^{\circ} \mathrm{C}$ or $1 \mathrm{~h}$ at RT. The next day, primary cultures were washed two times with $0.2 \%$ triton in PBS for 3 min and incubated with the secondary antibody (anti-rabbit Cy3, SigmaAldrich) for $1 \mathrm{~h}$ at RT and protected from light. To remove an excess of Cy3, cells were washed twice with $0.2 \%$ triton and two times in PBS, respectively. Coverslips with primary cultures were dried gently and mounted with a proper mounting medium (DakoCytomation). Neurons were visualized using a fluorescence microscope (Olympus BX51WI) and a 60x/0.90 objective (Olympus LUMPlanFI), and an excitation wavelength of $525 \mathrm{~nm}$.

\subsection{Optical recordings}

To perform dynamic and semi-quantitative recordings of redox changes specifically in neurons, the genetically-engineered optical redox probe roGFP1 was used. Native GFP protein became redox-sensitive by inserting two cysteines, between which a functional disulfide (S-S) bond forms, depending on the surrounding environmental oxidation/reduction conditions. These structural changes of roGFP1 modulate its absorption and fluorescence (Figure 6 A) (Hanson et al. 2004). The response principle of roGFP1 relies on two absorption peaks, which respond oppositely to redox modulation. Upon oxidation, the roGFP1 light absorption increases at $395 \mathrm{~nm}$ and decreases at $470 \mathrm{~nm}$, and this is the opposite upon reduction. Already in real time during the experiment, the roGFP1 fluorescence emission ratio $\left(F_{395} / F_{470}\right)$ is calculated (Figure $\left.6 \mathrm{~B}\right)$. Since individual neuronal cell bodies 
and/or parts of the dendrites were used as regions of interest, several cells within the field of view could be recorded at the same time. To visualise the dynamics of redox changes, neurons were imaged every $10 \mathrm{~s}$.

Alternatively, to assess cellular ROS/redox changes, also the geneticallyencoded hydroxyl peroxide sensor HyPer may be used. HyPer consists of circularly permuted YFP (cpYFP) linked to OxyR-RD - the regulatory domain of the $\mathrm{H}_{2} \mathrm{O}_{2}$ sensitive Escherichia coli transcription factor. However, it was suggested to be quite pH sensitive (Elsliger et al. 1999; Wachter et al. 2000; Schwarzländer et al. 2011). To monitor pH-mediate responses of HyPer, a plasmid vector carrying a modified redox-insensitive variant of HyPer SypHer (HyPer C199S) - was used, which only shows the artifactual pH responses associated with a given (redox challenging) treatment. To image SypHer responses, primary cell cultures were viewed by using a 60x 0.9 NA water immersion objective (LUMPlanFI; Olympus). The SypHer sensor was excited alternatively at $420 \mathrm{~nm}$ and $490 \mathrm{~nm}$, and the ratio of $420 \mathrm{~nm} / 490 \mathrm{~nm}$ was calculated.

To ensure dynamic live-imaging of primary cell and slice cultures in vitro, an optical recording set-up was used. It consisted of a suitable light source (Polychrome II; Till Photonics) and a sensitive CCD camera (Imago QE; PCO Imaging), which was fixed to the microscope. To perform optical recordings, cell and slice cultures were placed in a submersion-style chamber, which was constantly supplied with a pre-warmed ACSF at a solution flow rate of 4.5 $\mathrm{ml} / \mathrm{min}$. All recordings were performed by using the TILLvisION software and device-control package (version 4.0.1; TILL Photonics). 

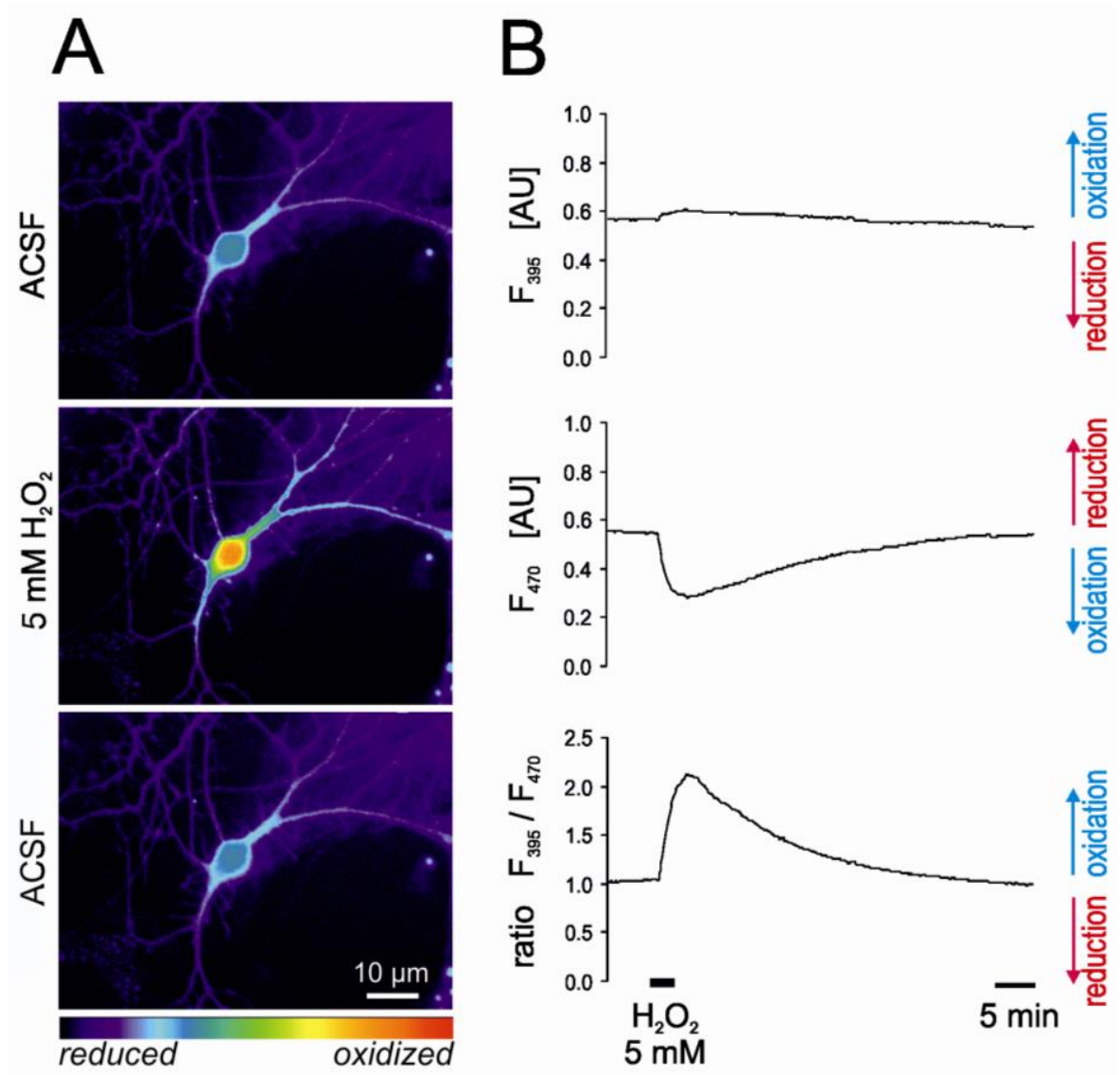

Figure 6. Ratiometric response properties of roGFP1.

A) Cytosol-targeted roGFP1 responded to oxidant challenge, which was induced by $\mathrm{H}_{2} \mathrm{O}_{2}$. In the displayed ratiometric images oxidation is indicated by "warm" pseudocolors and reduction by "cold" pseudocolors.

B) The redox sensor roGFP1 exhibits two absorption peaks, which show opposite changes upon redox modulation. Fluorescence excited at $395 \mathrm{~nm}\left(F_{395}\right)$ increased, while fluorescence excited at $470 \mathrm{~nm}\left(\mathrm{~F}_{470}\right)$ decreased under oxidation. As a result, the roGFP1 ratio $\left(\mathrm{F}_{395} / \mathrm{F}_{470}\right)$ increases upon oxidation.

\subsection{The 2-photon laser scanning microscope}

In order to visualize primary hippocampal neurons as well as organotypic hippocampal slices transduced with mito-roGFP1 and cyto-roGFP1 at an optical higher resolution, cell and slice cultures were imaged by a 2-photon laser scanning microscope (LaVision BioTec TriMScope II with BX51WI, Olympus). Excitation of roGFP1 was performed at a wavelength of $890 \mathrm{~nm}$, using a $63 \times 1.0$ NA objective (Zeiss Plan-Apochromat VIS-IR). The 3dimentional reconstructions of images were assembled with Metamorph Offline Software (version 6.1; Molecular Devices). 
To enable ratiometric imaging of the cyto-roGFP1 ratio in neurons from hippocampal acute slices of adult roGFP1c transgenic mice, the recently established dual laser-based ratiometric 2-photon excitation approach was used (Wagener et al. 2016). Alternating excitation of roGFP1c slices was performed at wavelengths $910 \mathrm{~nm}$ (provided by the MaiTai eHP laser system, Neuport-Spectra Physics) and $740 \mathrm{~nm}$ (provided by the Millenia-pumped Tsunami laser system, Neuport-Spectra Physics), and a 40x 0.8NA IRoptimized objective (IR Achroplan, Zeiss) was used.

\subsection{Local redox challenge}

To evaluate sub-cellular redox changes, primary neurons were transduced with the cyto-roGFP1 optical sensor. Patch clamp-shaped capillaries (GC150F-10 borosilicate glass, Harvard Part no. 30-0057, Harvard Apparatus) were mounted in a pipette holder of a 3-axis step micromanipulator (Luigs \& Neumann) and used for a local pressure puff application of redox stimulants. Tips of these capillaries were trimmed to a resistance of $\sim 5 \mathrm{M} \Omega$. Application of local stimuli was performed by the pneumatic drug ejection system (PDES-02D npi). Applied pressure was 50 mbar and stimulation of neurons was performed for various durations. In parallel, images of cells were taken every $2 \mathrm{~s}$.

\subsection{Electrical stimulation}

The extracellular stimulating electrode was made from the same type of capillaries used for pressure puff application. A chlorided silver wire (Science Products) was attached to a microconnector and connected to the stimulus isolation unit (PSIU-6, Grass Instrument Co.). The wire was inserted into the glass capillary, which was filled with ACSF. The stimulation electrode was mounted to the 3-axis step motor micromanipulator and carefully positioned close to a neuronal soma without actually touching the cell membrane.

In order to establish the electrical stimulation procedure, neurons were first loaded with a calcium indicator Fluo-3 AM (2 mM stock solution in DMSO, 
stored frozen at $-20^{\circ} \mathrm{C}$, Molecular Probes). To stain neuronal cultures in vitro, Fluo-3 AM $(5 \mu \mathrm{M})$ was added directly to growing medium with primary cell cultures and incubated for $30 \mathrm{~min}\left(37^{\circ} \mathrm{C}, 5 \% \mathrm{CO}_{2}\right)$. To induce any neuronal responses, cells were stimulated using the following parameters: $10-100 \mu \mathrm{A}$ current, $1 \mathrm{~s}$ stimulation only, $1 \mathrm{~ms}$ pulses at $1-5 \mathrm{~Hz}$ frequencies. Extracellular electrical stimulation was elicited by using a S88 stimulator (Grass Instruments). The Fluo-3 targeted neurons were visualised at a wavelength of $470 \mathrm{~nm}$.

For the comparison of the responses of WT and $M e c p 2^{-/ y}$ hippocampal neurons, primary neuronal cultures transduced with cyto-roGFP1 were used.

\subsection{Statistics}

For statistical calculations a two-tailed, unpaired Student $t$ test was used, with a significance level of $5 \%$. Significant changes have been indicated by asterisks ( $\left.{ }^{*} \mathrm{p}<0.05 ;{ }^{* *} \mathrm{p}<0.01 ;{ }^{* * *} \mathrm{p}<0.001\right)$. All values constitute mean \pm standard deviation (SD). The number of trials $(n)$ represents the number of cells taken for the experiments, unless stated otherwise. 


\section{Results}

The current thesis aimed to assess the potential redox changes in $M e c p 2^{-1 /}$ hippocampal primary neurons and organotypic hippocampal slices by taking advantage of genetically-encoded redox sensors. Local sub-cellular redox differences within single neurons were monitored in cytosol and mitochondria, and redox changes associated with neuronal activity were investigated. Also, the effects of various physiological stimuli on cellular redox homeostasis were compared among WT and $M e c p 2^{-1 y}$ neurons to define Rett syndrome associated differences in cellular redox balance.

\subsection{Challenge to select the ideal optical redox indicator}

Among the genetically-encoded redox sensors only HyPer and roGFPs are ratiometric by excitation, thereby enabling also quantitative analyses. The cytosol and mitochondria expressing plasmids of HyPer are commercially available and allow evaluating cellular redox dynamics within these two cellular compartments. The advantage of HyPer is that it detects $\mathrm{H}_{2} \mathrm{O}_{2}$ directly, which is not possible with other sensors, such as roGFPs, which report general thiol redox balance.

Unfortunately, chromophores based on YFP and cpYFP are $\mathrm{pH}$ sensitive (Elsliger et al. 1999; Wachter et al. 2000; Schwarzländer et al. 2011). Since $\mathrm{pH}$ may change during hypoxia and neuronal stimulation, the $\mathrm{pH}$-sensitivity of HyPer was assessed in order to decide, whether HyPer sensor is indeed suitable and sufficiently reliable for the planed experiments. To monitor $\mathrm{pH}$ changes, arising from various treatments and stimuli, a plasmid vector, which carries a modified variant of HyPer - SypHer - was used (Figure 7). It is no longer redox-sensitive but it shows only the artifactual $\mathrm{pH}$ responses. 


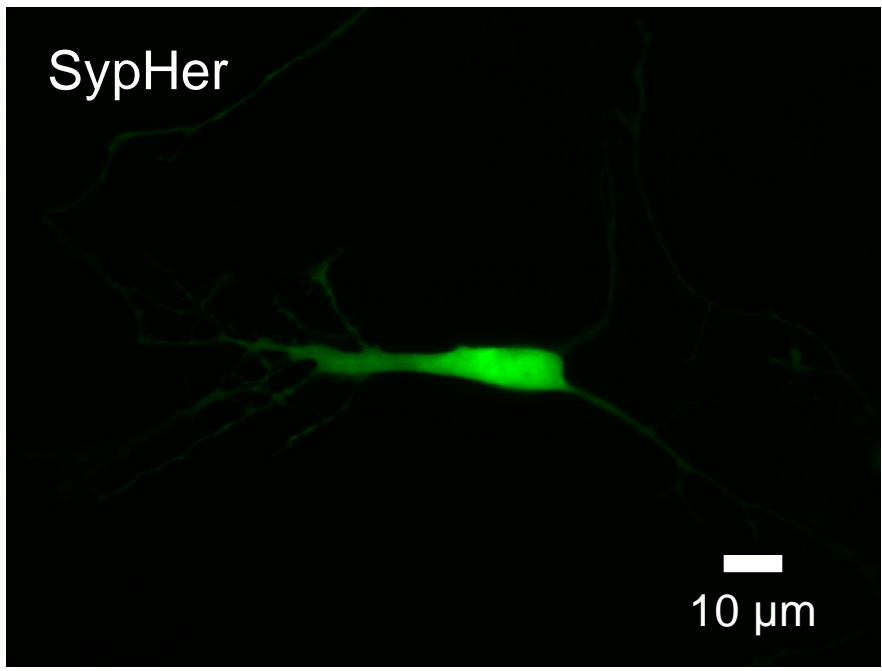

Figure 7. Hippocampal primary cell transfected with SypHer.

Cultured hippocampal cell expressing the cytosol-targeted SypHer sensor. Sufficient expression of SypHer was achieved within DIV 3-4. In accordance to HyPer, also SypHer exhibits two absorption peaks at $420 \mathrm{~nm}$ and $490 \mathrm{~nm}$.

\subsubsection{Calibration of the SypHer sensor}

To define the SypHer response kinetics as well as response magnitudes in different $\mathrm{pH}$ conditions, primary hippocampal cells were transfected with the SypHer vector and exposed to pre-warmed $\mathrm{pH}$-calibration solutions, starting from $\mathrm{pH} 7.0$ to $\mathrm{pH}$ 7.8. As soon as the cells had reached a stable baseline, the cellular membrane potential was disrupted by the $\mathrm{K}^{+} / \mathrm{H}^{+}$ionophore nigericin $(5 \mu \mathrm{M})$ and the SypHer ratio responses were recorded for $\sim 25 \mathrm{~min}$ (Figure 8 ). Once the cells reached a stable plateau, 10 successive images were averaged to calculate the final SypHer ratio. In these trials, a given cell was only exposed to one of the $\mathrm{pH}$ calibration solutions.

To record the entire response range, SypHer-transfected primary hippocampal cells were exposed successively to all $\mathrm{pH}$ solutions, applying each respective $\mathrm{pH}$ for $\sim 4 \min$ (Figure 9; Table 1). For reliable analyses, ratiometric calculations were corrected for cell movements in these trials. 


\begin{tabular}{|c|c|}
\hline $\mathbf{p H}$ & SypHer ratio $(\Delta \mathrm{R}$ SypHer $)$ \\
\hline 7.0 & $0.5 \pm 0.03 \%$ \\
\hline 7.2 & $0.7 \pm 0.02 \%$ \\
\hline 7.4 & $1.0 \pm 0.05 \%$ \\
\hline 7.6 & $1.5 \pm 0.09 \%$ \\
\hline 7.8 & $1.9 \pm 0.2 \%$ \\
\hline
\end{tabular}

Table 1. SypHer ratio changes induced by various $\mathrm{pH}$.

Listed are averages \pm SD of 7 cells.
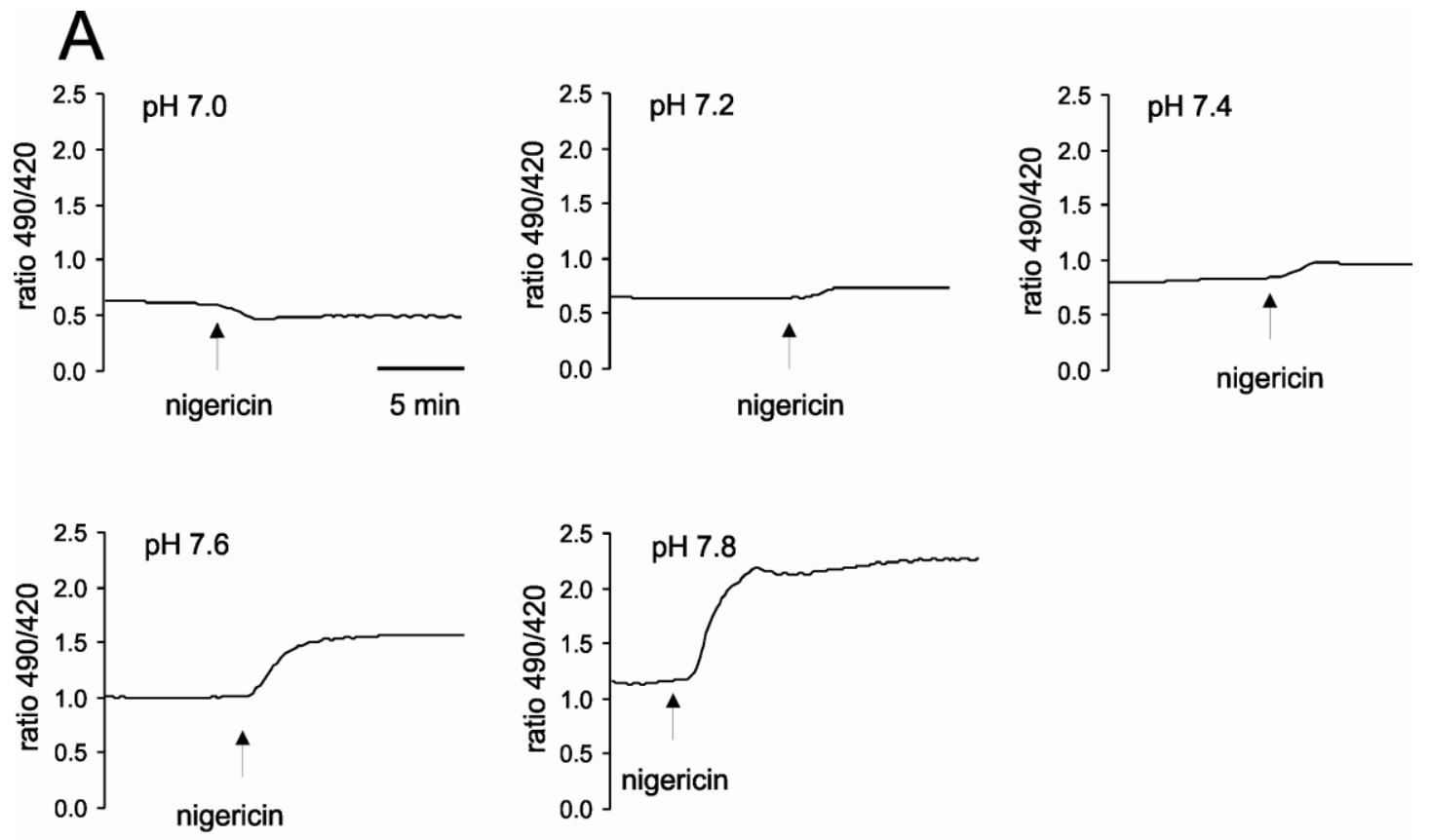

B

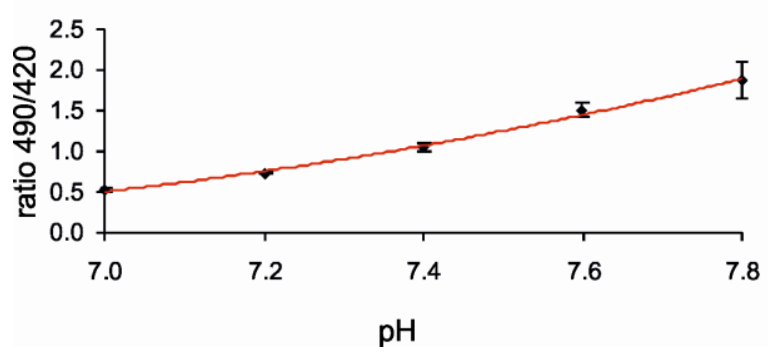

Figure 8. The pH-calibration of the SypHer sensor.

A) The SypHer ratio adjusts to current extracellular $\mathrm{pH}$ conditions within 1-2 min once the membrane integrity is challenged by nigericin. Time scaling applies to all traces.

B) Summary of the SypHer responses to different $\mathrm{pH}$ conditions. 


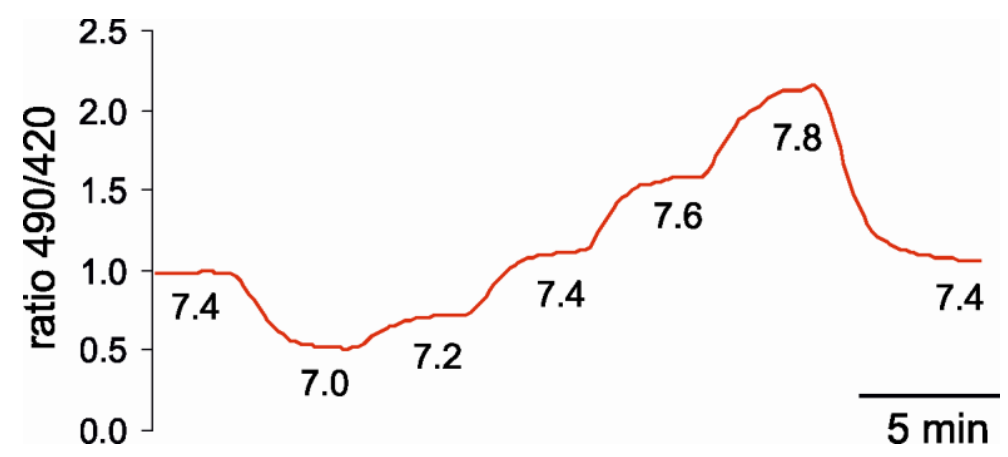

Figure 9. Full range calibration of SypHer recorded as an example in a single cell.

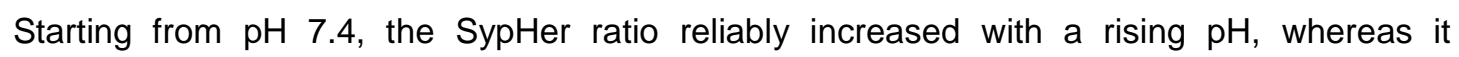
decreased when $\mathrm{pH}$ was lowered.

\subsubsection{Challenging SypHer by various redox stimuli}

The next set of experiments addressed $\mathrm{pH}$-changes resulting from e.g. redox stimuli, and they were recorded in cells with intact all membranes. Hippocampal cell cultures of WT mice were transfected with the cytosolic SypHer construct and exposed to $\mathrm{pH}$ challenge, which was induced by propionate $(30 \mathrm{mM})$. Also, different redox stimuli such as $\mathrm{H}_{2} \mathrm{O}_{2}(50 \mu \mathrm{M}$ and 5 $\mathrm{mM})$, tBHP $(20 \mu \mathrm{M})$, DEDTC $(50 \mu \mathrm{M}), \mathrm{CN}^{-}(1 \mathrm{mM})$, glutamate $(50 \mu \mathrm{M}, 1 \mathrm{mM})$, DTT $(10 \mathrm{mM})$ and hypoxia were applied. Hypoxic conditions were induced by applying the oxygen scavenger sodium sulfite $\left(\mathrm{Na}_{2} \mathrm{SO}_{3} ; 1 \mathrm{mM}, 15 \mathrm{~min}\right)$ and nitrogen aerated ACSF (95\% $\left.\mathrm{N}_{2} / 5 \% \mathrm{CO}_{2} ; 15 \mathrm{~min}\right)$. In accordance to the previous experiment, an increase of the SypHer fluorescence ratio indicates an alkalinization, whereas a decrease of the SypHer ratio corresponds to an acidification.

First of all, the responses of HyPer and SypHer were directly compared. In the case of HyPer, administration of propionate $(30 \mathrm{mM}, 15 \mathrm{~min}$ ) resulted in an initial and transient decrease of the HyPer ratio, which was followed by an increase when propionate was removed again (Figure $10 \mathrm{~A}$; Table 2). Upon the same treatment, the SypHer ratio showed a similar pattern of responses, indicating intracellular acidification and alkalinization, respectively (Figure 10 B; Table 2). This shows that a large part of the HyPer response simply does 
not represent redox changes but rather artifactual responses due to $\mathrm{pH}$ changes.
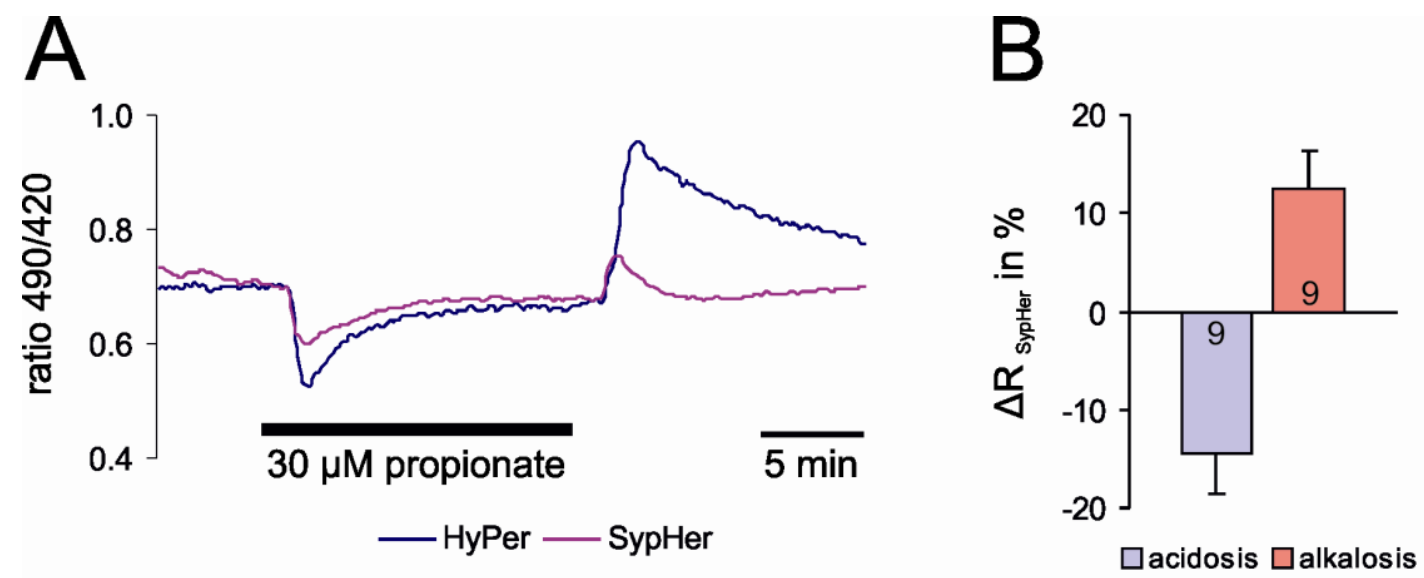

Figure 10. HyPer and SypHer responses to modulation of cellular $\mathrm{pH}$.

A) The HyPer ratio initially decreased and recovered back to baseline in the presence of propionate $(30 \mathrm{mM})$, and then increased upon wash out. Note that SypHer shows the same response pattern. The HyPer trace included here as a reference, was recorded already earlier by others (Weller et al. 2014).

B) The summary of the SypHer responses shows that application of propionate induced an acidification, which was then followed by an alkalinization.

\begin{tabular}{|c|c|c|}
\hline $\begin{array}{c}\text { Propionate } \\
(30 \mathrm{mM})\end{array}$ & $\begin{array}{c}\text { HyPer ratio } \\
\left(\Delta \mathrm{R}_{\mathrm{HyPer}}\right)\end{array}$ & $\begin{array}{c}\text { SypHer ratio } \\
\left(\Delta \mathrm{R}_{\text {SypHer }}\right)\end{array}$ \\
\hline Acidosis & $\begin{array}{c}-23.5 \pm 4.5 \% \\
n=8\end{array}$ & $\begin{array}{c}-14.5 \pm 4.2 \% \\
n=9\end{array}$ \\
\hline \multirow{2}{*}{ Alkalosis } & $\begin{array}{c}50.5 \pm 20.2 \% \\
n=8\end{array}$ & $\begin{array}{c}12.5 \pm 3.9 \% \\
n=9\end{array}$ \\
\hline
\end{tabular}

Table 2. The $\mathrm{pH}$ changes of redox-insensitive sensor SypHer.

Treatment with propionate ( $30 \mathrm{mM}, 15 \mathrm{~min}$ ) triggered $\mathrm{pH}$ changes, which can be measured as the SypHer ratio. However, these $\mathrm{pH}$ changes are also detected by the redox sensor HyPer, giving rise to non-reliable results. 
Administration of low $(50 \mu \mathrm{M})$ concentrations of $\mathrm{H}_{2} \mathrm{O}_{2}$ induced an increase in the SypHer ratio (Figure 11 A, Figure 13; Table 3). Interestingly, a short initial increase in the SypHer ratio, induced by a high dose $(5 \mathrm{mM})$ of $\mathrm{H}_{2} \mathrm{O}_{2}$, was followed by marked decrease. Oxidant challenge by tBHP (20 $\mu \mathrm{M}, 5$ min) revealed a marked alkalinization (Figure 11 B, Figure 13; Table 3). Also, treatment with DEDTC $(50 \mu \mathrm{M}, 10 \mathrm{~min})$ evoked a slight increase in the SypHer ratio (Figure 11 C, Figure 13; Table 3). Induction of reducing conditions by DTT (10 mM, $10 \mathrm{~min}$ ), also increased the SypHer ratio (Figure 11 D, Figure 13; Table 3). During hypoxia (15 min), the SypHer ratio was only slightly decreased and then it increased during reoxygenation (Figure $12 \mathrm{~A}$, Figure 13; Table 3). $\mathrm{CN}^{-}$(1 $\mathrm{mM}, 5 \mathrm{~min}$ ) elicited a biphasic response, an alkalinization followed by an acidification (Figure 12 B, Figure 13; Table 3). Pronounced acidification was induced by glutamate (50 $\mu \mathrm{M}, 3 \mathrm{~min})$, which was even more obvious upon application of a high dose of glutamate $(1 \mathrm{mM}$, 3 min) (Figure $12 \mathrm{C}$, Figure 13; Table 3).

A

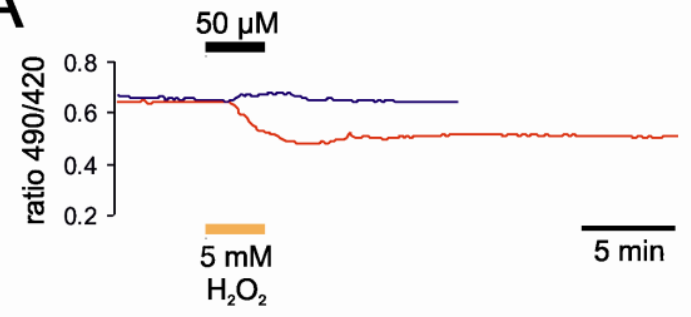

C

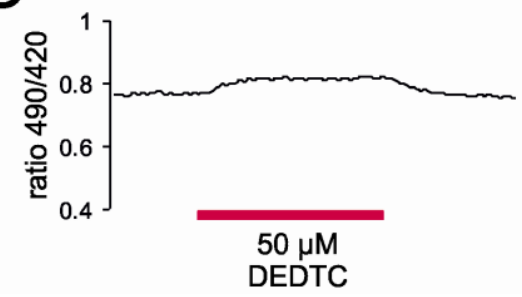

B

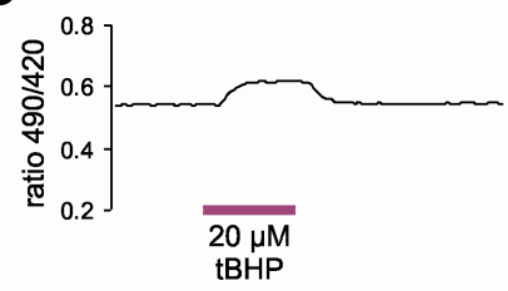

D

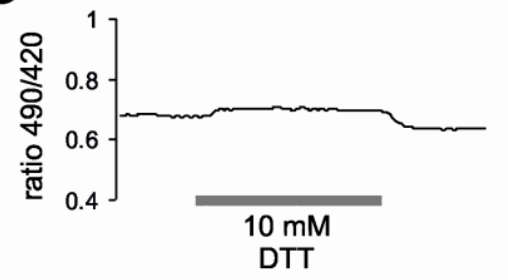

Figure 11. SypHer responses to redox challenge.

A) Low dosage $(50 \mu \mathrm{M})$ of $\mathrm{H}_{2} \mathrm{O}_{2}$ increased the SypHer ratio, while high concentrations (5 $\mathrm{mM}$ ) of $\mathrm{H}_{2} \mathrm{O}_{2}$ induced a pronounced acidification. All traces are displayed at the same time scaling.

B) Oxidant challenge provoked by tBHP revealed an increase in the SypHer ratio.

C) Alkalinization was evoked by DEDTC treatment.

D) The SypHer ratio revealed an increase upon reducing conditions induced by DTT. 
A

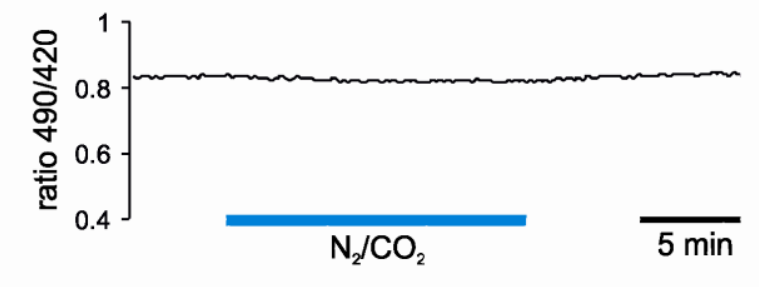

C

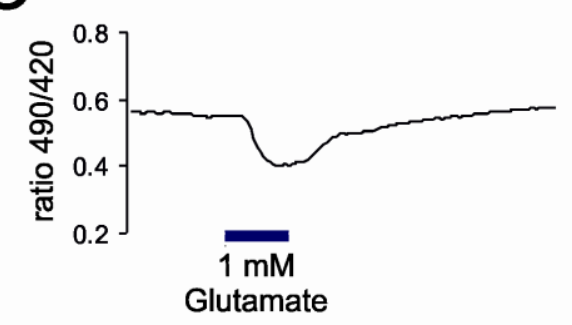

B

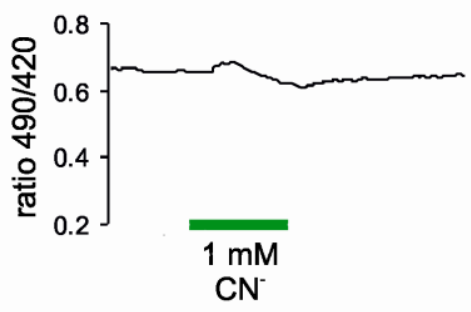

Figure 12. SypHer responses to hypoxia and excitotoxicity.

A) The SypHer ratio was only slightly decreased during hypoxia. This was then followed by a minor increase upon reoxygenation. All traces are displayed at the same time scaling.

B) Chemically-induced hypoxia by $\mathrm{CN}^{-}$caused a short and reversible alkalinization followed by an acidificaion.

C) Administration of glutamate elicited a reversible and pronounced acidification.

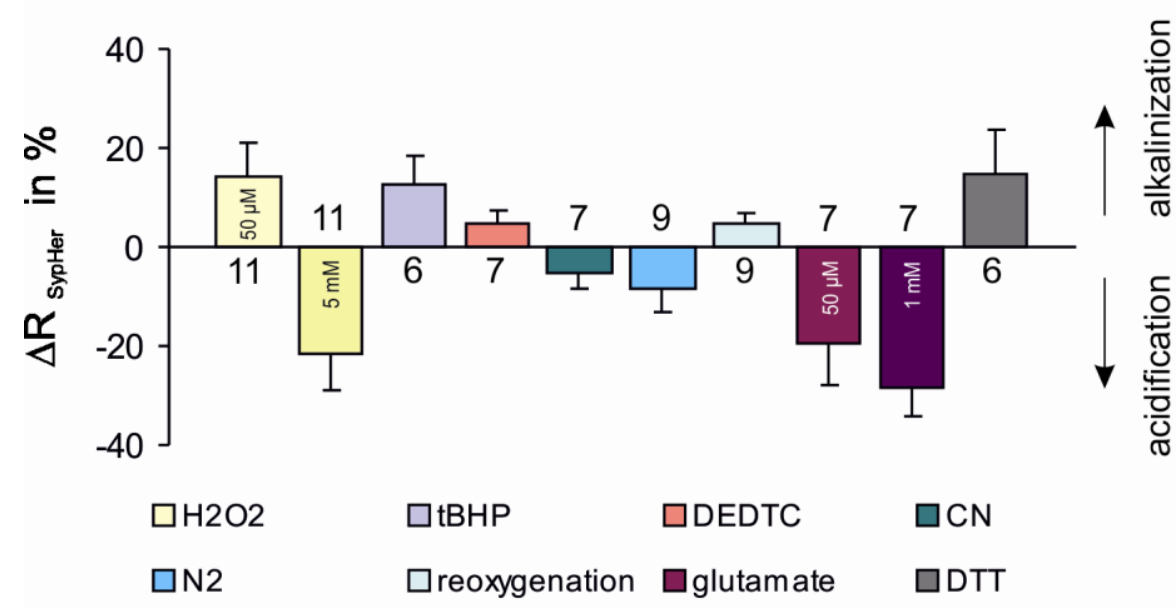

Figure 13. Summary of SypHer responses to changes in redox balance.

The SypHer ratio responded with an acidification during treatments with $\mathrm{H}_{2} \mathrm{O}_{2}(5 \mathrm{mM}), \mathrm{CN}$, $\mathrm{N}_{2}$ and glutamate. Administration of $\mathrm{H}_{2} \mathrm{O}_{2}(50 \mu \mathrm{M})$, tBHP, DEDTC, reoxygenation upon hypoxia and DTT evoked alkalinization. 


\begin{tabular}{|c|c|c|}
\hline & \multicolumn{2}{|c|}{ SypHer ratio $\left(\Delta \mathrm{R}_{\text {SypHer }}\right)$} \\
\hline Treatment & Acidosis & Alkalosis \\
\hline $\mathrm{H}_{2} \mathrm{O}_{2}(50 \mu \mathrm{M})$ & - & $\begin{array}{c}14.4 \pm 6.9 \% \\
n=11\end{array}$ \\
\hline $\mathrm{H}_{2} \mathrm{O}_{2}(5 \mathrm{mM})$ & $\begin{array}{c}-21.5 \pm 7.6 \% \\
n=11\end{array}$ & $\begin{array}{c}6.4 \pm 4.5 \% \\
n=11\end{array}$ \\
\hline tBHP $(20 \mu \mathrm{M})$ & - & $\begin{array}{c}12.9 \pm 5.6 \% \\
n=6\end{array}$ \\
\hline DEDTC $(50 \mu \mathrm{M})$ & - & $\begin{array}{c}4.8 \pm 2.8 \% \\
n=7\end{array}$ \\
\hline $\mathrm{CN}^{-}(1 \mathrm{mM})$ & $\begin{array}{c}-5.2 \pm 3.3 \% \\
n=7\end{array}$ & $\begin{array}{c}3.2 \pm 1.2 \% \\
n=7\end{array}$ \\
\hline Hypoxia & $\begin{array}{c}-8.3 \pm 4.6 \% \\
n=9\end{array}$ & $\begin{array}{c}4.7 \pm 2.2 \% \\
n=9\end{array}$ \\
\hline Glutamate $(50 \mu \mathrm{M})$ & $\begin{array}{c}-19.6 \pm 8.2 \% \\
n=7\end{array}$ & - \\
\hline Glutamate (1 mM) & $\begin{aligned}-28.4 & \pm 5.9 \% \\
n & =7\end{aligned}$ & - \\
\hline DTT (10 mM) & - & $\begin{array}{c}14.9 \pm 8.5 \% \\
n=6\end{array}$ \\
\hline
\end{tabular}

Table 3. The pH changes of SypHer sensor upon various redox stimuli.

The $\mathrm{pH}$-sensitive, redox-insensitive optical sensor SypHer showed very clear $\mathrm{pH}$ changes, indicating acidosis and/or alkalosis, which were associated with the different redox stimuli.

\subsection{Redox changes in primary neuronal cell culture and organotypic slice culture}

Since the HyPer sensor has been found not to be a suitable redox indicator as its responses were markedly disturbed by $\mathrm{pH}$ changes, the geneticallyencoded roGFP1 sensor was used for all further experiments. For roGFP1 it has been confirmed earlier that it is hardly affected by $\mathrm{pH}$ changes (Funke et al. 2011). 
To asses any changes in the redox homeostasis specifically within neurons, primary cell and slice cultures were transduced with a neuron-specific viral expression vector AAV-6, carrying roGFP1 and targeting it to mitochondria (mito-roGFP1) as well as the cytosol (cyto-roGFP1). At first, proper expression of roGFP1 in cytosolic and mitochondrial compartments of cell and organotypic culture preparations was confirmed (Figure 14). Since roGFP1 gives excitation ratiometric responses, variations in redox balance could be measured and analysed quantitatively.

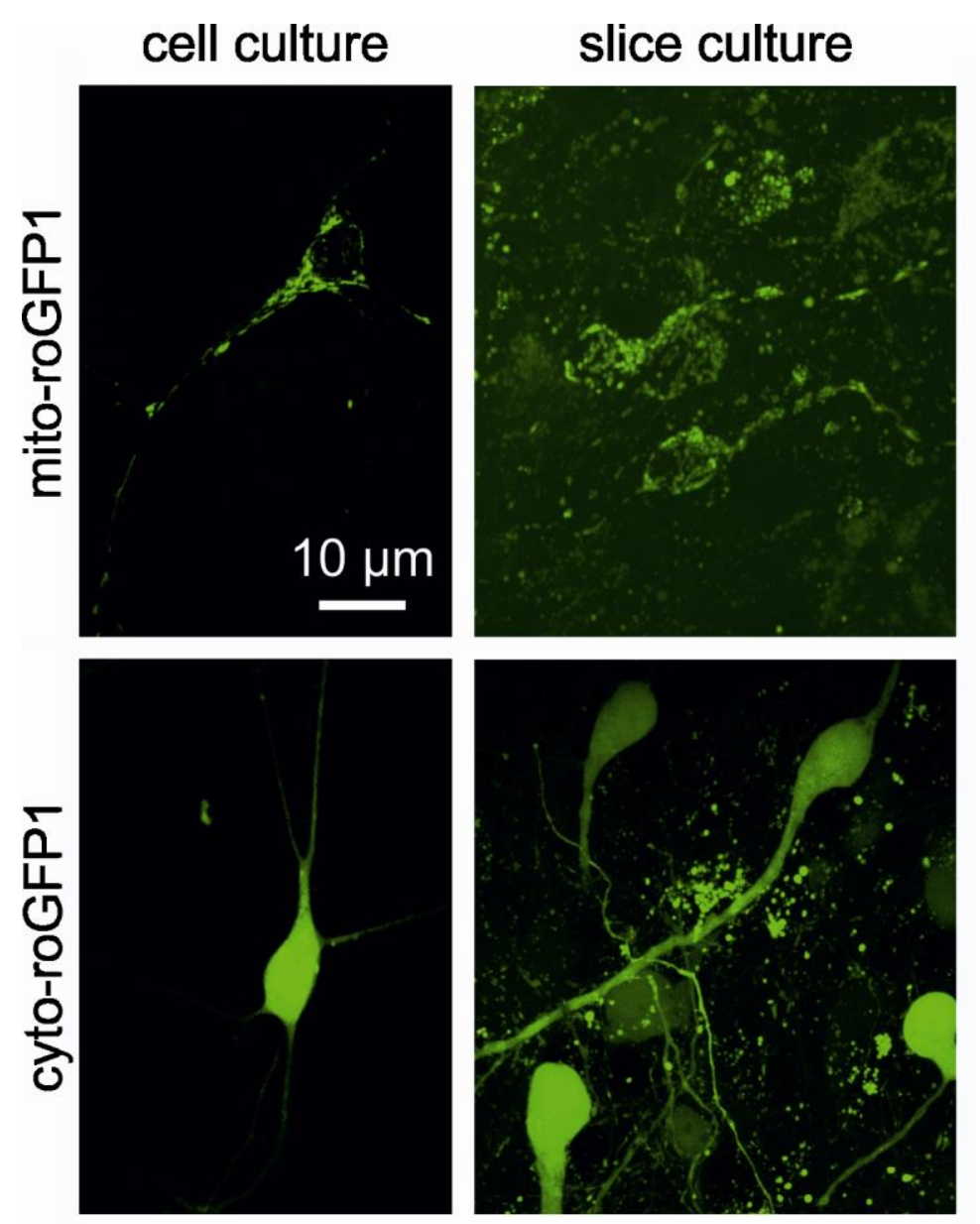

Figure 14. Transduction of primary cell and slice cultures with cyto-roGFP1 and mito-roGFP1.

Viral transduction ensured neuron-specific expression of roGFP1. Sufficient expression levels were obtained within DIV 6-7. Mito-roGFP1 reported the redox changes in mitochondrial matrix of individual mitochondria in neuronal cell cultures and organotypic slice cultures. Cyto-roGFP1 was distributed homogenously in cytosol, reporting redox conditions in the cytosolic sub-compartments. The scale bar applies to all images displayed. 


\subsubsection{Confirming proper sub-cellular localization of mito- and cyto-roGFP1 in neurons}

The mitochondria-specific synthetic dye MitoTracker RED FM was used to certify specific targeting of mito-roGFP1 to intact mitochondria of primary neurons (Figure $15 \mathrm{~A}-\mathrm{C}$ ). To confirm the specific targeting of cyto-roGFP1 to neuronal cells, primary cell cultures were fixed and stained with MAP2directed antibody as MAP2 is present only in neurons, in their perikarya and dendrites in particular (Figure $15 \mathrm{D}-\mathrm{F}$ ).

A

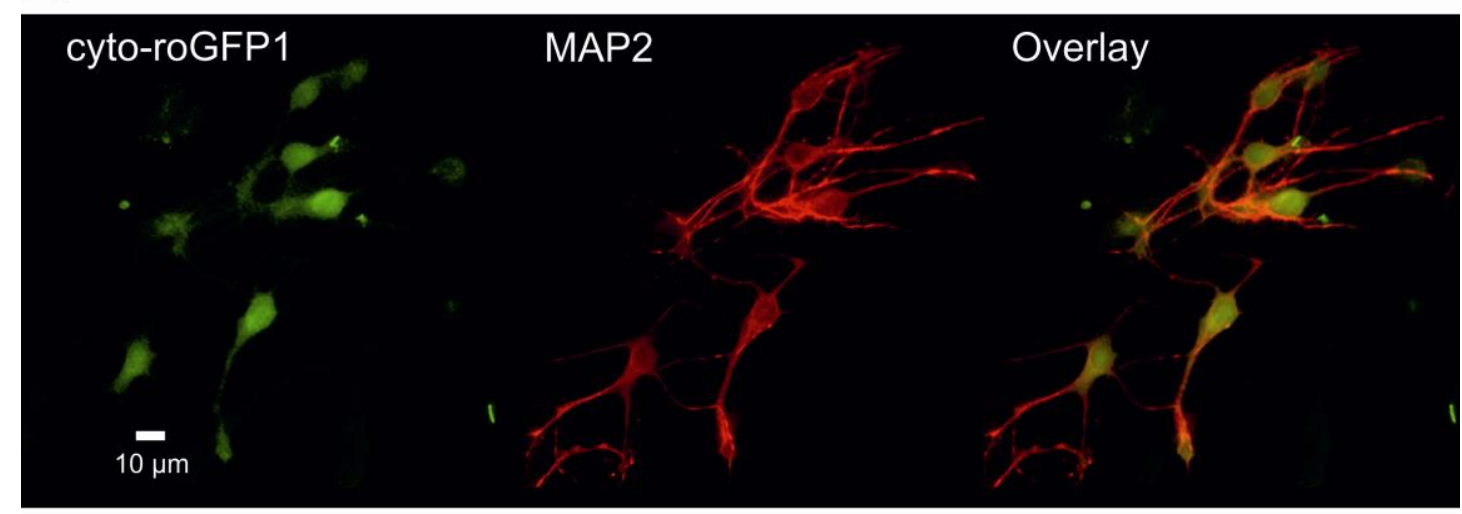

B

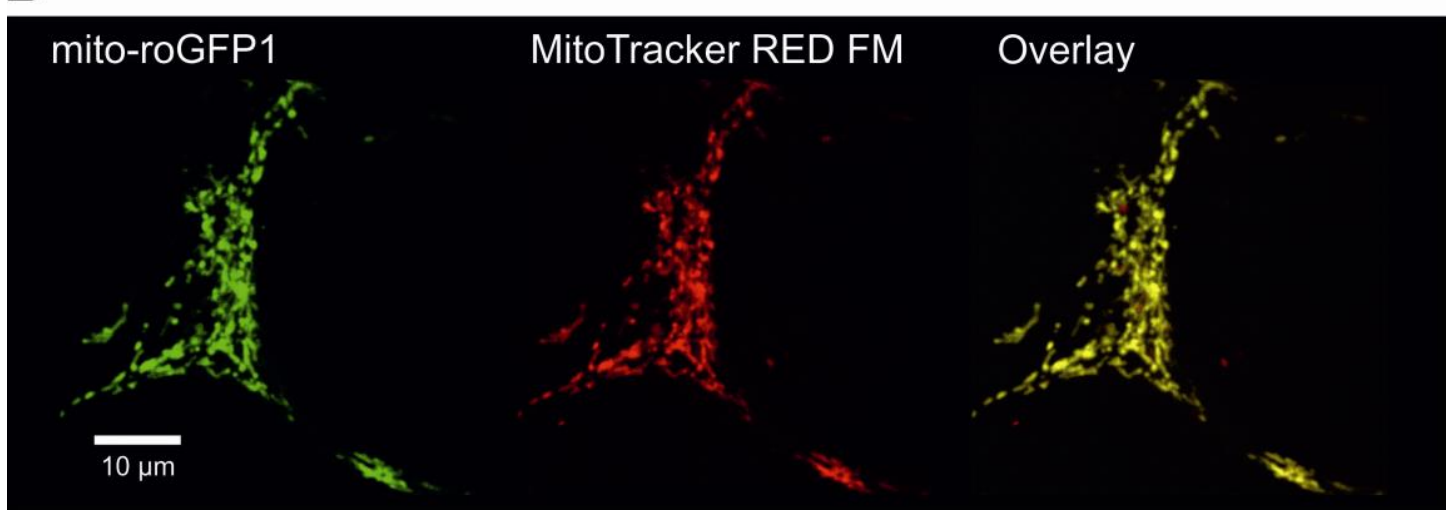

Figure 15. Sub-cellular localization of mito-roGFP1 and cyto-roGFP1.

A) Cyto-roGFP1 was expressed homogenously within hippocampal neurons as confirmed by MAP2 immunolabeling. The scale bar applies to all images.

B) Mito-roGFP1 specifically targeted mitochondrial compartments in neurons. MitoTracker RED FM confirmed the proper location of mito-roGFP1 in neuronal mitochondria. 


\subsubsection{Calibration of cyto- and mito-roGFP1}

To perform semi-quantitative analyses, the ratiometric fluorescence responses of mito-roGFP1 and cyto-roGFP1 were calibrated to maximum oxidation and reduction. Complete oxidation was induced by applying saturating doses of $\mathrm{H}_{2} \mathrm{O}_{2}(5 \mathrm{mM}, 3 \mathrm{~min})$ and full reduction was obtained by DTT (10 mM, $3 \mathrm{~min}$ ) (Hanson et al. 2004; Funke et al. 2011; Großer et al. 2012). According to the assumption of linear roGFP1 responses, the relative amount of oxidation/reduction of the respective construct was estimated (Figure $16 \mathrm{~A}$ ), and $\mathrm{Mecp2}^{-1 /}$ and WT genotypes as well as primary cell culture and organotypic slice culture preparations could be compared. Calibrations were performed for each sensor construct as well as in each type of in vitro preparation. Both genotypes responded similarly to the calibration treatments. However, the response ranges of mito-roGFP1 in Mecp2 $2^{-1 y}$ and WT neurons in slice cultures were somewhat decreased in comparison to cultured dissociated cells (Figure 16 B; Table 4).
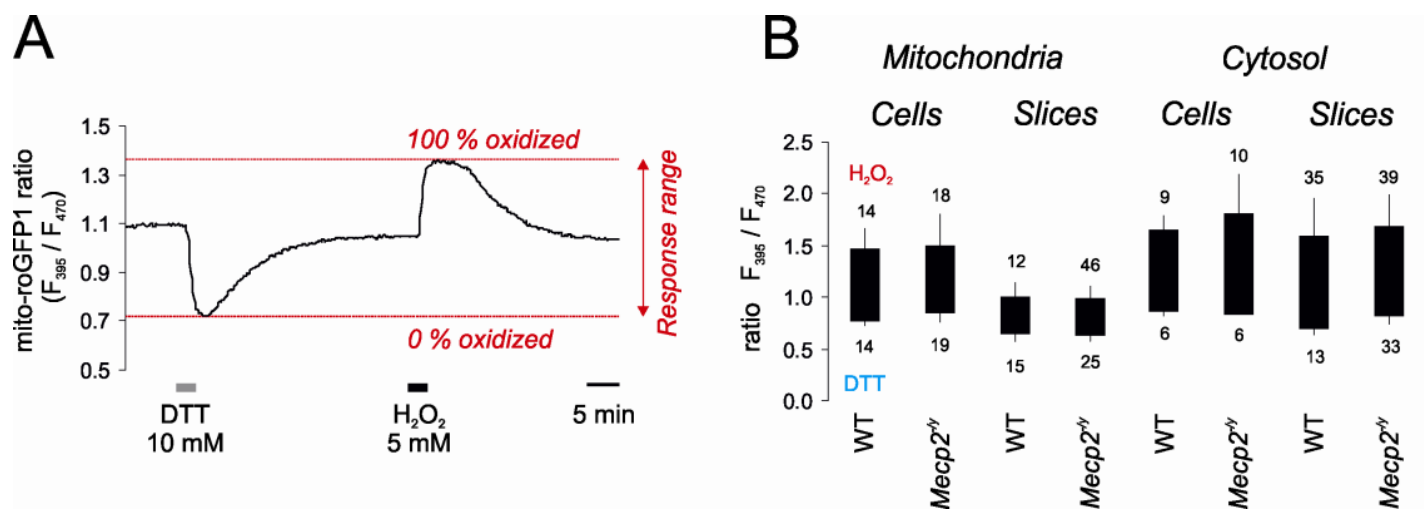

Figure 16. Response range of mito-roGFP1 and cyto-roGFP1.

A) Calibration of mito-roGFP1 responses in a hippocampal neuron to full oxidation and reduction. Upon reduction, induced by DTT, the fluorescence of mito-roGFP1 decreased and it increased upon oxidation, which was mediated by $\mathrm{H}_{2} \mathrm{O}_{2}$.

B) Calculated response ranges of mito-roGFP1 and cyto-roGFP1 in primary cell and organotypic hippocampal slice cultures. 


\begin{tabular}{|c|c|c|c|c|}
\hline & & \multicolumn{3}{|c|}{ roGFP1 ratio $\left(F_{395} / F_{470}\right)$} \\
\hline & & WT & Mecp2 $2^{-/ y}$ & Treatment \\
\hline \multirow{4}{*}{ 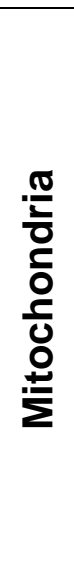 } & \multirow{2}{*}{ Cell culture } & $\begin{array}{c}1.5 \pm 0.2 \\
n=14\end{array}$ & $\begin{array}{c}1.5 \pm 0.3 \\
n=18\end{array}$ & $\mathrm{H}_{2} \mathrm{O}_{2}$ \\
\hline & & $\begin{array}{c}0.7 \pm 0.1 \\
n=14\end{array}$ & $\begin{array}{c}0.8 \pm 0.1 \\
n=19\end{array}$ & DTT \\
\hline & \multirow{2}{*}{ Slice culture } & $\begin{array}{c}1.0 \pm 0.1 \\
n=12\end{array}$ & $\begin{array}{c}1.0 \pm 0.1 \\
n=46\end{array}$ & $\mathrm{H}_{2} \mathrm{O}_{2}$ \\
\hline & & $\begin{array}{c}0.6 \pm 0.1 \\
n=15\end{array}$ & $\begin{array}{c}0.6 \pm 0.1 \\
n=25\end{array}$ & DTT \\
\hline \multirow{4}{*}{ 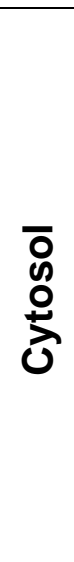 } & \multirow{2}{*}{ Cell culture } & $\begin{array}{c}1.7 \pm 0.1 \\
n=9\end{array}$ & $\begin{array}{c}1.8 \pm 0.4 \\
n=10\end{array}$ & $\mathrm{H}_{2} \mathrm{O}_{2}$ \\
\hline & & $\begin{array}{c}0.8 \pm 0.1 \\
n=6\end{array}$ & $\begin{array}{c}0.8 \pm 0.0 \\
n=6\end{array}$ & DTT \\
\hline & \multirow{2}{*}{ Slice culture } & $\begin{array}{c}1.6 \pm 0.4 \\
n=35\end{array}$ & $\begin{array}{c}1.7 \pm 0.3 \\
n=39\end{array}$ & $\mathrm{H}_{2} \mathrm{O}_{2}$ \\
\hline & & $\begin{array}{c}0.6 \pm 0.1 \\
n=13\end{array}$ & $\begin{array}{c}0.7 \pm 0.1 \\
n=33\end{array}$ & DTT \\
\hline
\end{tabular}

Table 4. Calibration of the roGFP1 redox sensors in mitochondrial and cytosolic compartments.

Listed are the peaks and nadirs recorded in response to oxidation and reduction, respectively.

\subsubsection{The roGFP1 pre-treatment baseline ratio in mitochondria and cytosol}

Previously, it has been shown that the roGFP1 pre-treatment baseline ratio was significantly increased in the cytosol of neonatal Mecp2 $2^{-y}$ hippocampi (Großer et al. 2012). In these trials the roGFP1-coding DNA was delivered by lipofectamine, which certainly resulted in a mixed population of roGFP1 expressing neurons and glial cells. In the present thesis, redox conditions were monitored specifically in neurons, and analyses were not only performed in the cytosol, but also extended to the mitochondrial matrix. This was done in a comparable fashion in WT and $M e c p 2^{-/ y}$ hippocampal primary neurons as 
well as in organotypic slice cultures. The specific delivery of roGFP1 to neurons was achieved by the AAV-6 viral vector, which mediated a highly efficient and specific roGFP1 expression under the control of the human synapsin I promoter.

Differing redox conditions in WT and $M e c p 2^{-/ y}$ mice were especially obvious in organotypic slice cultures under control conditions. In the cultured hippocampal tissue, Mecp2 ${ }^{-/ y}$ neurons revealed more oxidized roGFP1 baseline ratios both in their mitochondria $(p<0.05)$ and in cytosol $(p<0.001)$ than WT neurons. Significant changes were also observed not only between WT and Mecp2 $^{-l y}$ genotypes, but also between mitochondrial and cytosolic compartments. Interestingly, the roGFP1 pre-treatment baseline ratio was significantly $(p<0.001)$ decreased in the cytosol of neuronal cultures from WT and $M e c p 2^{-/ y}$ hippocampus as compared to mitochondria. In contrast, it was markedly $(p<0.001)$ increased in cytosol as compared to mitochondria in organotypic slice cultures of these both genotypes (Figure 17; Table 5).

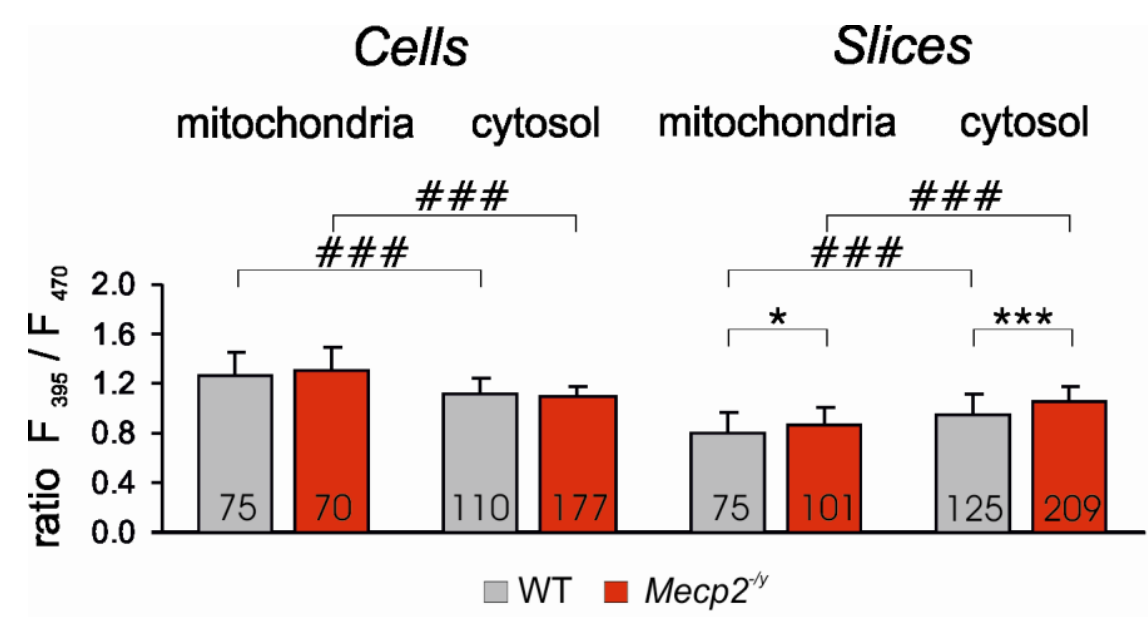

Figure 17. The cytosolic and mitochondrial pre-treatment baseline ratio responses.

In all dissociated cell cultures no differences of the cytosolic and mitochondrial roGFP1 baseline ratio between WT and Mecp2 ${ }^{-1 /}$ neurons were observed. However, in organotypic slice cultures, the roGFP1 baseline ratio was significantly increased in mitochondria $(p<0.05)$, and in cytosol of $(p<0.001) M^{-1 y}$ mice as compared to WT. Also, the roGFP1 baseline was significantly $(p<0.001)$ decreased in cytosol compared to mitochondria in dissociated cell cultures, which was opposite in organotypic slices. 


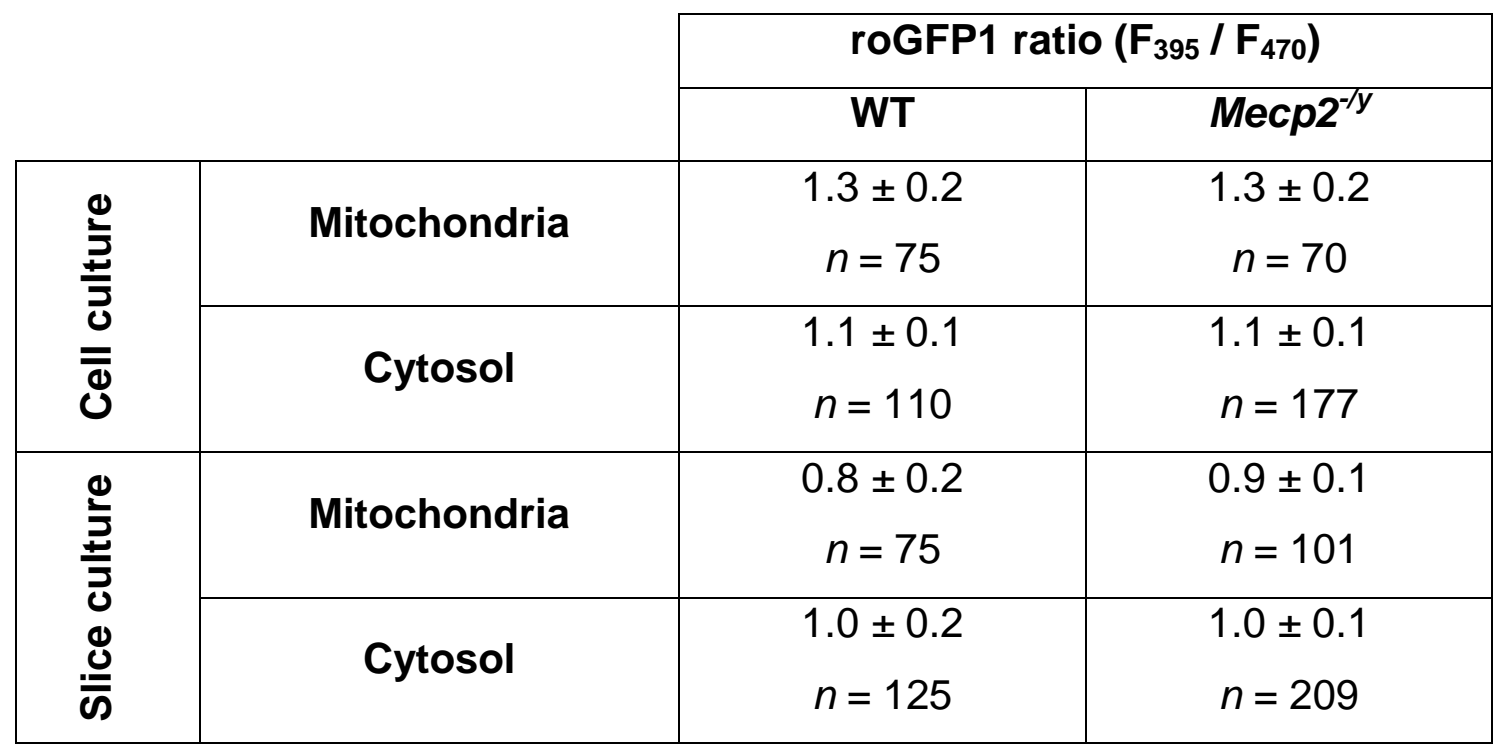

Table 5. Mito-roGFP1 and cyto-roGFP1 ratio baselines under control conditions in WT and $M e c p 2^{-/ y}$ neurons.

\subsubsection{Mitochondrial and cytosolic redox status}

In order to screen for redox changes of $M \operatorname{ccp}^{-/ y}$ hippocampus during hypoxia, oxygen $\left(\mathrm{O}_{2}\right)$ was removed by applying the $\mathrm{O}_{2}$ scavenger sodium sulfite $\left(\mathrm{Na}_{2} \mathrm{SO}_{3} ; 1 \mathrm{mM}, 10 \mathrm{~min}\right)$ together with $\mathrm{ACSF}$, which was aerated with nitrogen (95\% $\mathrm{N}_{2} / \mathrm{CO}_{2} ; 10 \mathrm{~min}$ ) (Figure $18 \mathrm{~A}$ ). To assess any alterations in the redox homeostasis of $\mathrm{Mecp2}^{-1 /}$ hippocampal neurons, hippocampal cell and slice cultures were challenged by acute oxidative stress, induced by a transient application of $\mathrm{H}_{2} \mathrm{O}_{2}(200 \mu \mathrm{M}, 3 \mathrm{~min})$ (Figure $18 \mathrm{~B}$ ). The efficiency of cellular scavenging systems in $M c p 2^{-/ y}$ neurons was rated by SOD scavenging enzyme inhibition, which was triggered by DEDTC ( $5 \mathrm{mM}, 5 \mathrm{~min}$ ) (Figure $18 \mathrm{C}$ ). Also, changes in mitochondrial ROS production were provoked by blocking the oxidative phosphorylation with the complex III inhibitor AMC $(20 \mu \mathrm{M}, 10 \mathrm{~min})$ (Figure $18 \mathrm{D})$. 

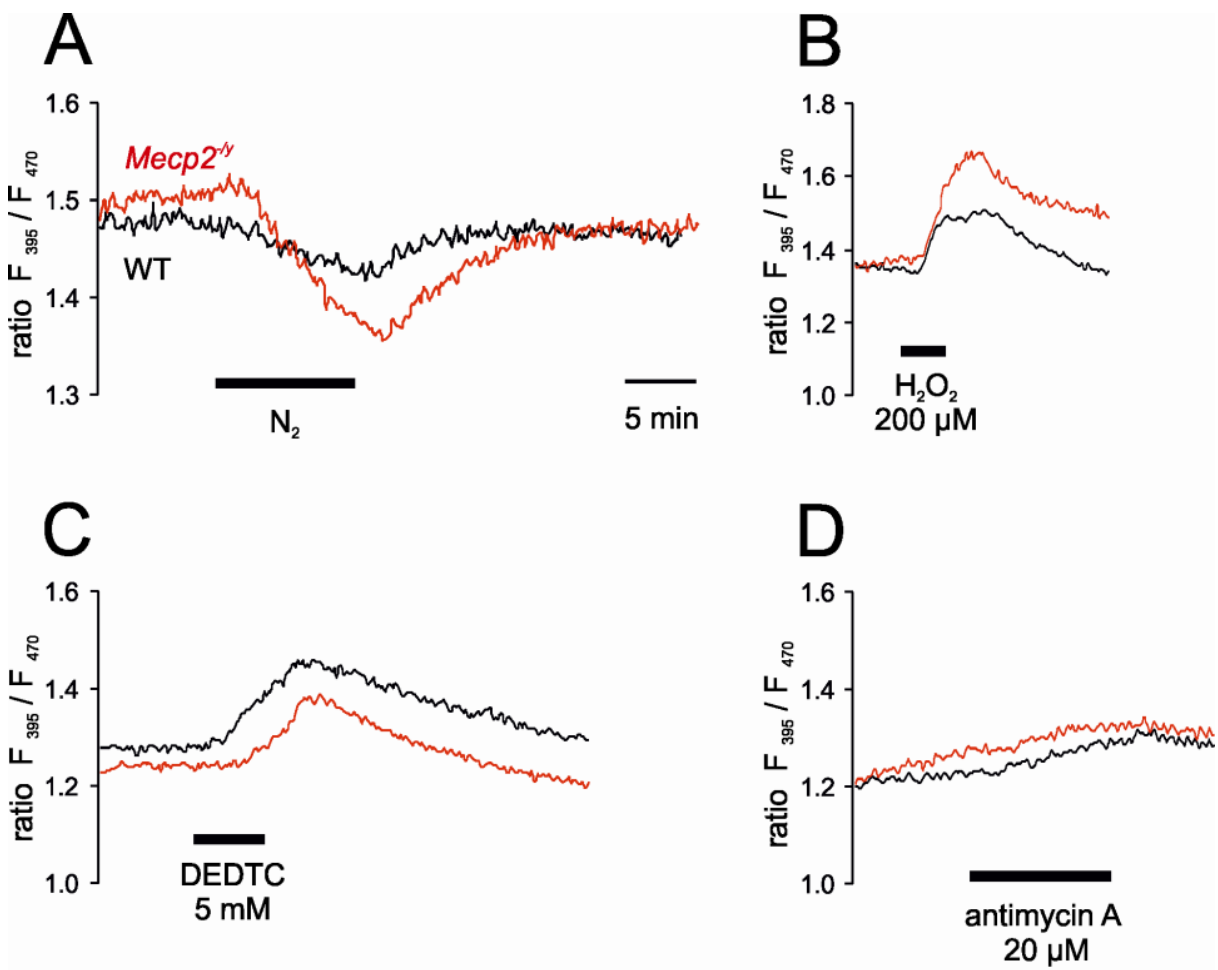

Figure 18. Rating of cellular redox homeostasis.

A) Severe hypoxia was induced by $\mathrm{O}_{2}$ withdrawal $\left(95 \% \mathrm{~N}_{2} / \mathrm{CO}_{2}\right)$ in the presence of the $\mathrm{O}_{2}$ scavenger $\mathrm{Na}_{2} \mathrm{SO}_{3}$. The roGFP1 ratio decreased towards more reducing conditions in $M_{\text {ecp }}{ }^{-/ y}$ hippocampus as compared to WT. The scale bar applies to all panels.

B) Direct oxidative challenge by $\mathrm{H}_{2} \mathrm{O}_{2}$ induced a clearly more intense oxidation in mitochondria of $\mathrm{Mecp2}^{-1 / y}$ mice as compared to WT.

C) Inhibition of SOD by DEDTC evoked a less intense oxidation in $M e c p 2^{-/ y}$ neurons than in WT.

D) Inhibition of mitochondrial electron transport chain by administration of AMC induced a slight oxidation, however, differences between WT and $M e c p 2^{-1 y}$ were not obvious.

Upon redox challenge, more obvious differences between WT and Mecp2-/y mice were detectable in hippocampal organotypic slice culture rather than in cell culture preparations. These changes were seen in both mitochondrial and cytosolic compartments (Figure 19).

In primary cell cultures, upon induction of hypoxia, the mito-roGFP1 ratio clearly $(\mathrm{p}<0.01)$ shifted towards more reducing conditions in $M e c p 2^{-/ y}$ neurons as compared to WT (Figure $19 \mathrm{~A}$; Table 6). However, application of $\mathrm{H}_{2} \mathrm{O}_{2}$, DEDTC and AMC did not reveal any significant differences among mitochondria of WT and Mecp2-/y neurons. Also, the cyto-roGFP1 ratio did not 
show any differences between WT and $M e c p 2^{-/ y}$ neurons during application of hypoxia, DEDTC and AMC (Figure $19 \mathrm{~B}$; Table 6). Only $\mathrm{H}_{2} \mathrm{O}_{2}$ evoked a shift to more $(p<0.05)$ oxidizing conditions in $M e c p 2^{-/ y}$ neurons as compared to WT.

Exaggerated redox responses were, however, found in organotypic slice cultures. During hypoxia, the mito-roGFP1 ratio reached more reducing levels in $M e c p 2^{-/ y}$ slices as compared to WT $(p<0.05)$. Also, the mito-roGFP1 ratio clearly $(p<0.01)$ shifted further towards oxidation in $M e c p 2^{-/ y}$ slices due to $\mathrm{H}_{2} \mathrm{O}_{2}$ application as compared to WT. Treatment with DEDTC evoked a less intense $(p<0.05)$ oxidation of mito-roGFP1 in $M e c p 2^{-/ y}$ slices as compared to WT. Moreover, the mito-roGFP1 ratio tended to increase more upon application of AMC in Mecp2 ${ }^{-/ y}$ slices as compared to WT (Figure $19 \mathrm{C}$; Table $6)$.

Pronounced differences in the redox responses between WT and Mecp2-/y slices were especially seen in the cytosol. Hypoxic conditions elicited clearly $(p<0.01)$ more reducing responses in $M e c p 2^{-/ y}$ slices as compared to WT. Redox challenge, induced by $\mathrm{H}_{2} \mathrm{O}_{2}$, resulted in a significantly $(\mathrm{p}<0.05)$ more intense oxidation in $M e c p 2^{-/ y}$ slices as compared to WT. Furthermore, the cyto-roGFP1 ratio was notably $(p<0.001)$ less effected in $M e c p 2^{-/ y}$ slices due to SOD inhibition as compared to WT. Surprisingly, blockade of the mitochondrial transport chain by AMC did not evoke any obvious differences in the cytosolic responses of WT and Mecp2 $2^{-/ y}$ slices (Figure $19 \mathrm{D}$; Table 6). 

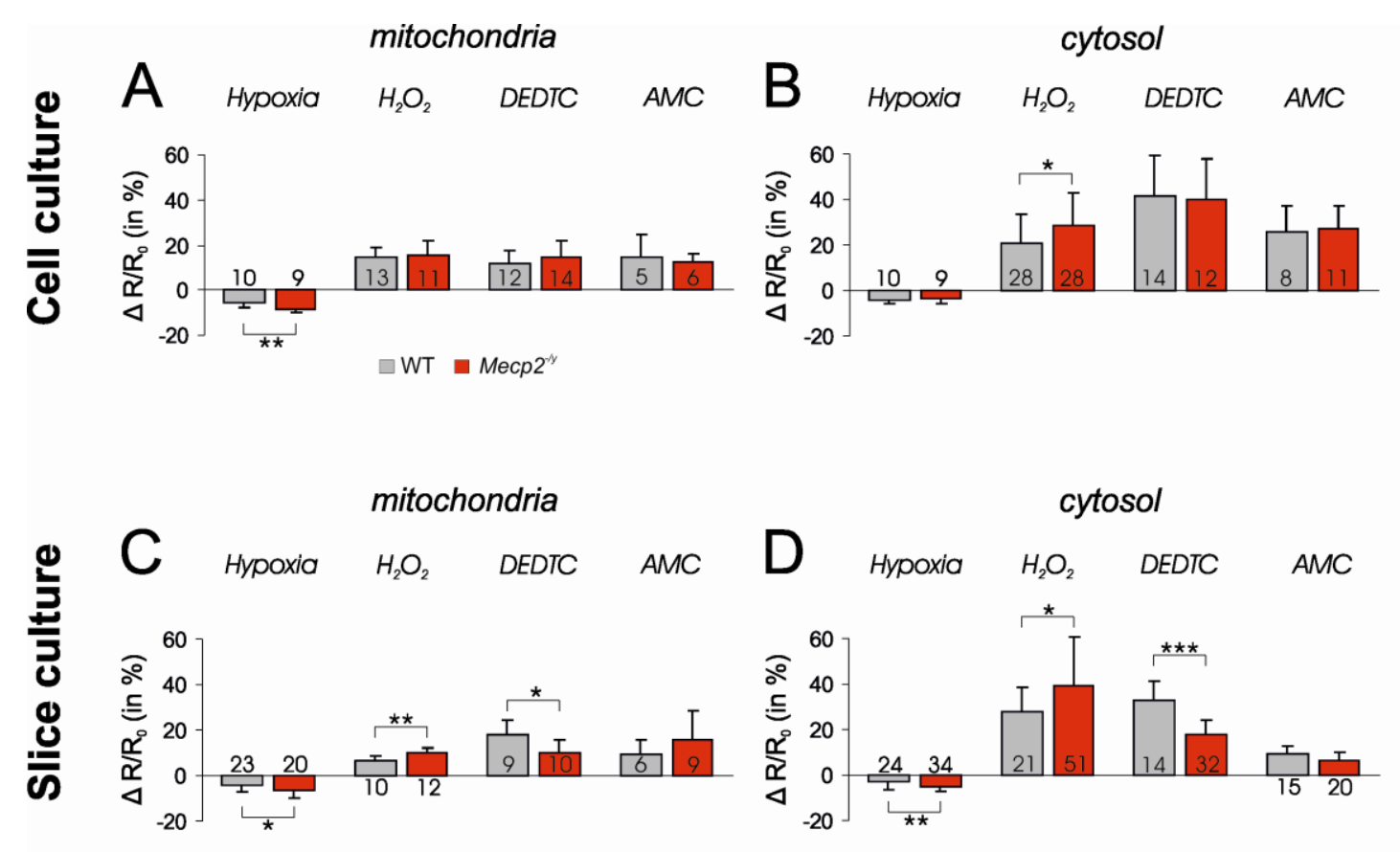

\section{Figure 19. Summary of redox responses in mitochondria and cytosol.}

A) Mito-roGFP1 ratio did not respond differently between WT and $M e c p 2^{-/ y}$ mice, when $\mathrm{H}_{2} \mathrm{O}_{2}$, DEDTC and AMC were applied. However, Mecp2 $2^{-1 y}$ neurons were more $(p<0.01)$ susceptible to hypoxia when compared to WT.

B) Mecp2 $2^{-1 y}$ and WT neurons responded equally to hypoxia as well as to DEDTC and AMC. Yet, the cyto-roGFP1 ratio shifted towards more $(p<0.05)$ oxidizing conditions in Mecp2 $2^{-y}$ neurons due to $\mathrm{H}_{2} \mathrm{O}_{2}$ treatment as compared to WT.

C) The mito-roGFP1 ratio shifted towards more $(\mathrm{p}<0.05)$ reducing conditions during hypoxia and showed more $(p<0.01)$ exaggerated responses to $\mathrm{H}_{2} \mathrm{O}_{2}$ in Mecp $2^{-/ y}$ organotypic slices as compared to WT. Moreover, DEDTC evoked a shift of the mito-roGFP1 ratio towards oxidation, which was less $(\mathrm{p}<0.05)$ intense in $M e c p 2^{-/ y}$ organotypic slices as compared to WT. Also, Mecp2 ${ }^{-/ y}$ slices showed a tendency towards more intense oxidation upon AMC administration as compared to WT.

D) Cyto-roGFP1 showed significantly lowered $(p<0.01)$ reducing responses during hypoxia and markedly $(p<0.05)$ increased oxidation in response to $\mathrm{H}_{2} \mathrm{O}_{2}$ in $M e c p 2^{-1 y}$ organotypic slices as compared to WT. Also, a weaker $(p<0.001)$ shift of the cyto-roGFP1 ratio towards oxidation was found in $M e c p 2^{-/ y}$ slice cultures due to DEDTC administration as compared to WT. However, no obvious differences between WT and Mecp2 ${ }^{-1 y}$ organotypic slices were observed when AMC was applied. 


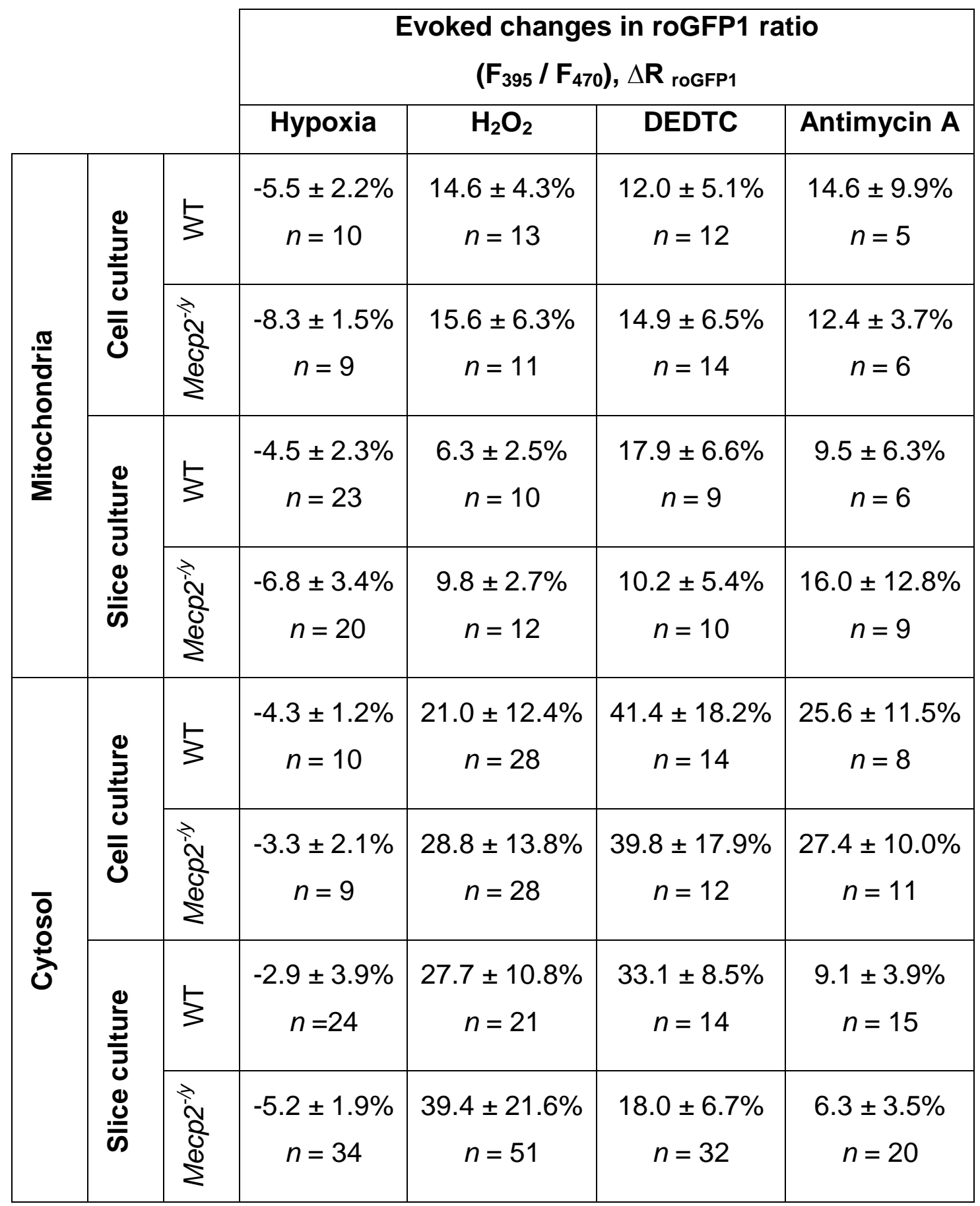

Table 6. Overview of the mito-roGFP1 and cyto-roGFP1 ratio responses in cultured neurons and hippocampal organotypic slices upon various redox stimuli. 


\subsection{Sub-cellular redox changes under control conditions and upon oxidative challenge.}

To investigate the redox conditions of somata and dendrites in $M e c p 2^{-1 y}$ neurons under control conditions, cyto-roGFP1 transduced neurons were imaged and analyzed for sub-cellular differences in their steady state roGFP1 ratios. For this, a line of interest (LOI) was drawn along the neurons, starting from the centre of the somata up to the periphery of single dendrites (Figure $20 \mathrm{~A})$. For statistical calculations, redox ratios were normalized to the mean of first ten pixels of the respective LOI. Furthermore, to reduce pixel noise, a moving average ( 5 data points wide) was applied. These analyses revealed a more increased cyto-roGFP1 baseline ratio in somata than in dendrites in WT and $M e c p 2^{-/ y}$ neurons (Figure $20 \mathrm{~B}$ ). Genotypic differences in this sub-cellular redox gradient were not observed.
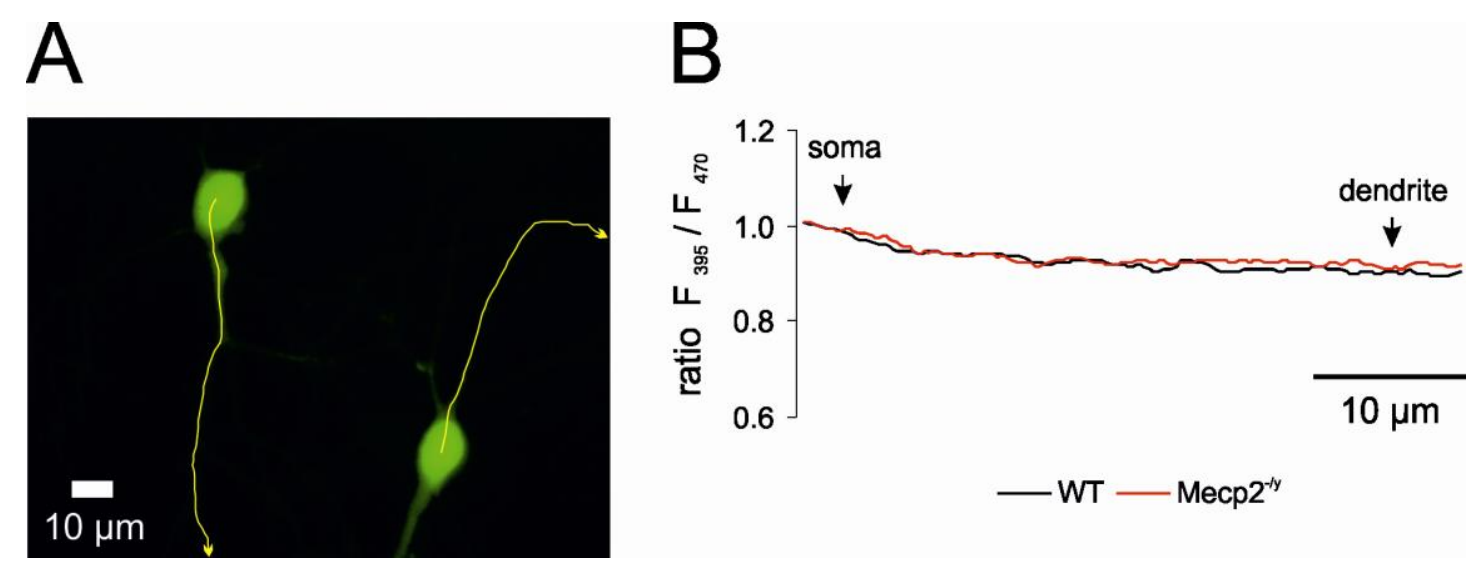

Figure 20. Oxidation level changes between somata and dendrites in WT and Mecp2 $^{-/ y}$ neurons under control conditions.

A) To analyze redox baselines in somatic and dendritic areas of hippocampal neurons, a LOI was drawn along a neuron from the middle of the soma into a well pronounced dendrite.

B) The cyto-roGFP1 ratio showed an increased steady state oxidation in somata, which was progressively decreasing towards the periphery of the dendrites.

To assess any differences in the redox responses to oxidative challenge between somatic and dendritic areas of $M e c p 2^{-1 / y}$ and WT neurons, $\mathrm{H}_{2} \mathrm{O}_{2}(200$ $\mu \mathrm{M}, 3 \mathrm{~min}$ ) was applied to cyto-roGFP1-transduced cell cultures. As a result, the cyto-roGFP1 ratio shifted towards more $(p<0.01)$ oxidizing conditions in somata than in dendrites of WT and $M e c p 2^{-1 y}$ neurons (Figure 21; Table 7). 
As expected, upon $\mathrm{H}_{2} \mathrm{O}_{2}$ treatment, the cyto-roGFP1 ratio increased also more intensively in $M e c p 2^{-/ y}$ neurons than in WT, and this was observed in neuronal dendrites $(p<0.001)$ and somata $(p<0.05)$.

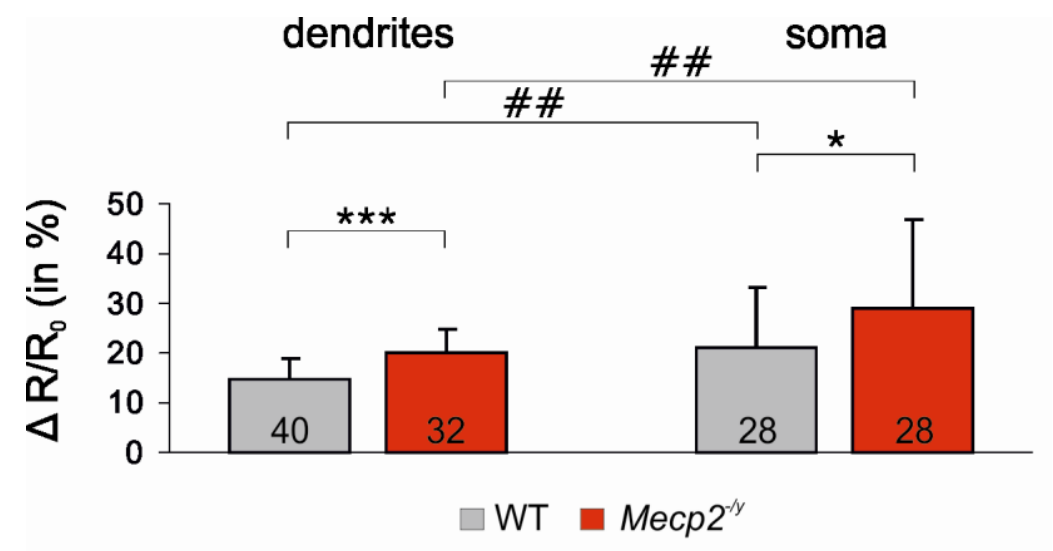

Figure 21. Local redox changes in response to oxidation challenge.

Upon $\mathrm{H}_{2} \mathrm{O}_{2}(200 \mu \mathrm{M}, 3 \mathrm{~min})$ application, more increased $(\mathrm{p}<0.01)$ cyto-roGFP1 ratios were apparent in the somata of WT and $M e c p 2^{-1 / y}$ neurons than in their dendrites. Furthermore, $M e c p 2^{-1 y}$ neurons showed significantly higher shifts of the cyto-roGFP1 ratios towards oxidation in dendrites $(p<0.001)$ and in soma $(p<0.05)$ as compared to WT.

\begin{tabular}{|c|c|c|}
\cline { 2 - 3 } \multicolumn{1}{c|}{} & \multicolumn{2}{c|}{$\begin{array}{r}\text { Evoked changes in roGFP1 ratio } \\
\left(\mathbf{F}_{395} / \mathbf{F}_{\mathbf{4 7 0}}\right), \Delta \mathbf{R}_{\text {roGFP1 }}\end{array}$} \\
\hline $\mathbf{H}_{\mathbf{2}} \mathbf{O}_{\mathbf{2}}(\mathbf{2 0 0} \boldsymbol{\mu} \mathrm{M})$ & $\mathbf{W T}$ & $\mathbf{M e c p 2}^{-/ \mathbf{y}}$ \\
\hline Dendrites & $\begin{array}{c}14.6 \pm 4.4 \% \\
n_{\text {dendrites }}=40\end{array}$ & $\begin{array}{c}19.9 \pm 4.9 \% \\
n_{\text {dendrites }}=32\end{array}$ \\
\hline Soma & $21.0 \pm 12.4 \%$ & $28.8 \pm 13.5 \%$ \\
& $n=28$ & $n=28$ \\
\hline
\end{tabular}

Table 7. Relative changes in the cyto-roGFP1 ratio in somatic and dendritic areas of WT and Mecp2 $^{-/ y}$ neurons upon oxidative challenge.

Usually, 1-2 dendrites were analyzed per cell.

To evaluate the changes in SOD efficiency specifically among soma and dendrites of $\mathrm{Mecp}^{-/ y}$ neurons, the cyto-roGFP1-transduced neuronal cultures were also treated with DEDTC ( $5 \mathrm{mM}, 5 \mathrm{~min})$ in order to inhibit SOD. Upon 
such treatment, Mecp2 $2^{-/ y}$ neuronal dendrites showed more increased $(p<0.01)$ cyto-roGFP1 ratios when compared to WT. Moreover, the cyto-roGFP1 ratio shifted towards more oxidation in somata than in dendrites, which was especially pronounced $(p<0.001)$ in WT (Figure 22; Table 8).

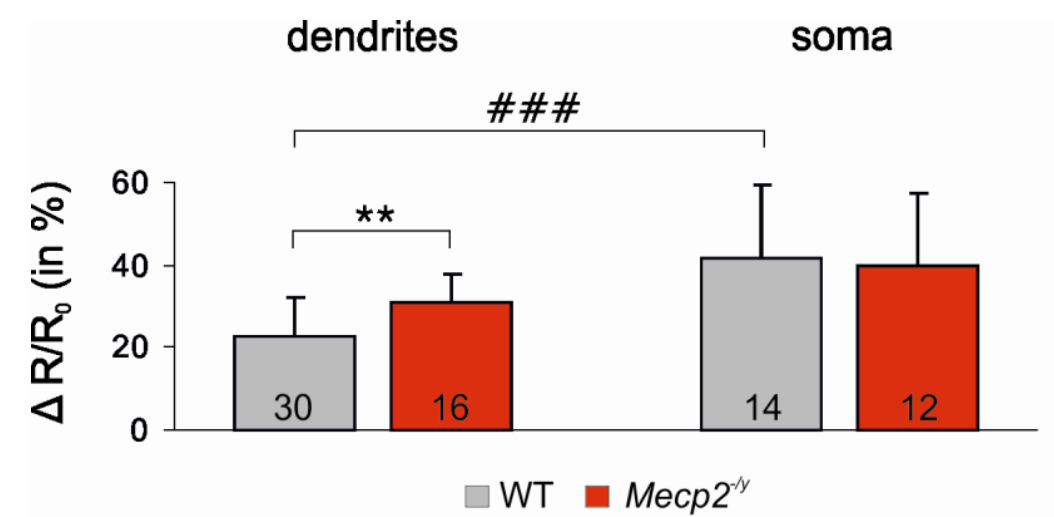

Figure 22. Local neuronal changes in response to SOD inhibition.

Upon SOD blockade, Mecp2 $2^{-1 y}$ dendrites showed a marked $(p<0.01)$ oxidizing shift in the cyto-roGFP1 ratio as compared to WT. Furthermore, more oxidized $(p<0.001)$ responses were observed in the somatic areas of WT neurons than in their somata.

\begin{tabular}{|c|c|c|}
\cline { 2 - 3 } \multicolumn{1}{c|}{} & \multicolumn{2}{c|}{$\begin{array}{r}\text { Evoked changes in roGFP1 ratio } \\
\left(\mathbf{F}_{395} / \mathbf{F}_{\mathbf{4 7 0}}\right), \Delta \mathbf{R}_{\text {roGFP1 }}\end{array}$} \\
\hline DEDTC (5 $\mathbf{~ m M )}$ & $\mathbf{W T}$ & $\mathbf{M e c p 2}^{-/ y}$ \\
\hline \multirow{2}{*}{ Dendrites } & $22.5 \pm 9.6 \%$ & $30.7 \pm 7.0 \%$ \\
& $n_{\text {dendrites }}=30$ & $n_{\text {dendrites }}=16$ \\
\hline \multirow{2}{*}{ Soma } & $41.4 \pm 18.2 \%$ & $39.8 \pm 17.9 \%$ \\
& $n=14$ & $n=12$ \\
\hline
\end{tabular}

Table 8. Differences in oxidation levels of somatic and dendritic areas between Mecp2 $^{-1 y}$ and WT neurons in response to DEDTC.

Usually, 1-2 dendrites were analyzed per cell. 


\subsection{Local redox stimulation of primary neurons}

In order to better understand the cellular redox physiology, it is crucial to challenge neurons with different redox stimuli. However, since neurons constitute a group of very sensitive and energy demanding cells, too high drug concentrations and/or long neuronal stimulation might contribute to cellular impairment, and result in unreliable observations. It is therefore important also to apply more subtle and localized stimuli. Hence, the next set of experiments aimed to establish the local neuronal stimulation by using a pneumatic drug ejection system, and to assess the sub-cellular changes in the roGFP1 ratio of $M e c p 2^{-/ y}$ neurons in response to oxidative challenge.

First, tests of the drug ejection system were performed to confirm proper and reproducible ejection of small volumes from a pipette tip once a pressure was applied. A glass pipette was filled with the highly fluorescing dye rhodamine 123 (Rh123), which was then ejected either in a constant or pulsed (100 ms, $5 \mathrm{~Hz}$ ) mode for durations of $5 \mathrm{~s}$ and $10 \mathrm{~s}$. Applied pressure was $20 \mathrm{mbar}$, and both single and repeated pressure puff stimulations resulted in a reliable Rh123 release (Figure 23).

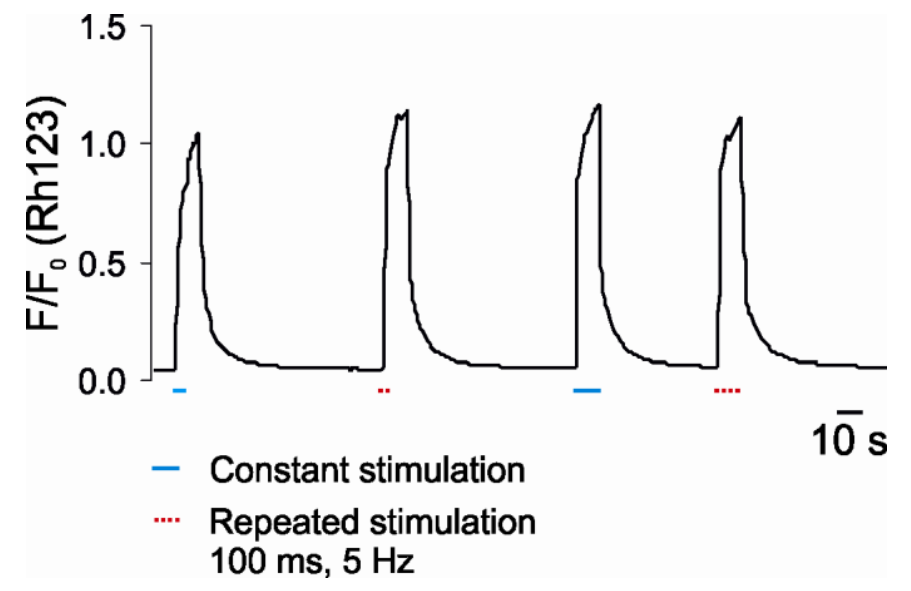

Figure 23. Rh123 release from a pipette tip by using pulsed puff stimuli.

Application of single (blue mark) and pulsed (red dashed mark) pressure puffs reliably induced Rh123 release from the pipette into the recording chamber. Plotted fluorescence was recorded with a region of interest placed in front of the pipette tip at a distance $\sim 5 \mu \mathrm{m}$.

Next, to rule out any unwanted mechanical effects that might appear upon puff stimulation of neurons, the pipette was filled with ACSF and positioned in 
close proximity to a dendrite of a cyto-roGFP1-transduced neuron (Figure 24 A) without actually touching the cell membrane (Figure 24 B). Single puffs with durations of $100 \mathrm{~ms}, 200 \mathrm{~ms}$ and $300 \mathrm{~ms}$ and $50 \mathrm{mbar}$ pressure were applied. No changes were observed in the cyto-roGFP1 ratio by such local ACSF puffs (Figure $24 \mathrm{C}$ ). Now, to redox challenge hippocampal neurons, a glass pipette was filled with $\mathrm{H}_{2} \mathrm{O}_{2}(5 \mathrm{mM})$ and placed closely to a proximal dendrite and the soma. The cell body was stimulated by pressure puffs in a repeated (100 ms, $5 \mathrm{~Hz}$ ) mode for $10 \mathrm{~s}, 30 \mathrm{~s}$ and $1 \mathrm{~min}$. Upon local $\mathrm{H}_{2} \mathrm{O}_{2}$ puffs, the cyto-roGFP1 ratio clearly increased and recovered to the pretreatment baseline (Figure $24 \mathrm{D})$.

A

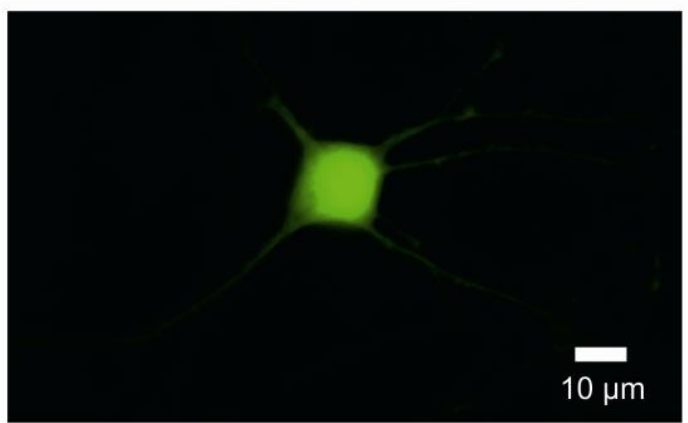

C

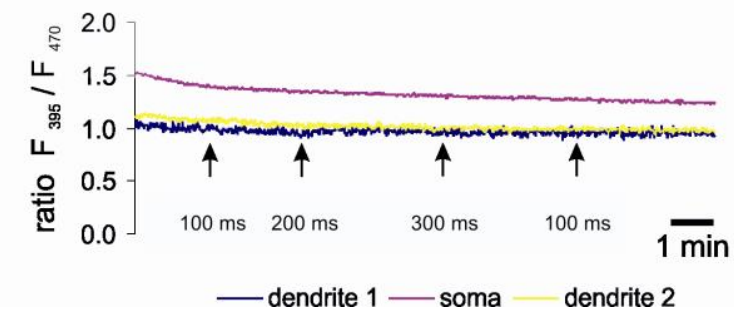

B

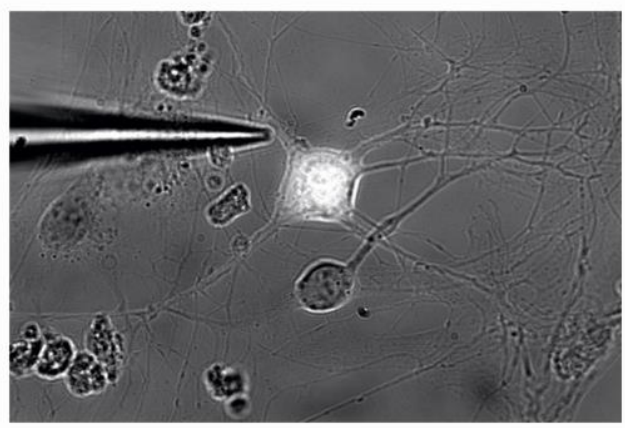

$\mathrm{D}$

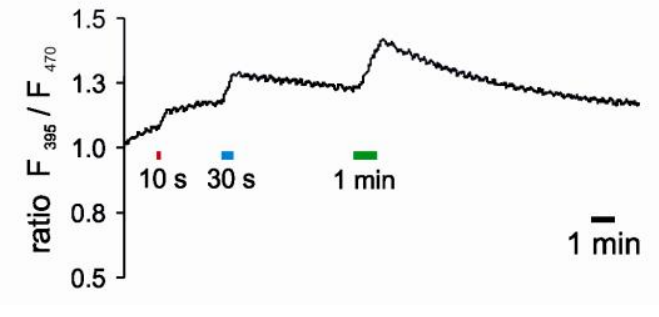

Figure 24. Local redox stimulation of a cyto-roGFP1-transduced neuron.

A) Cyto-roGFP1 transduced neurons were used for local redox stimulation. The scale bar applies to both images displayed.

B) The patch pipette was positioned in close proximity to a neuronal dendrite of a cytoroGFP1 transduced neuron, allowing therefore for a localized redox challenging.

C) When neurons were exposed to ACSF pressure puffs for $100 \mathrm{~ms}, 200 \mathrm{~ms}$ and $300 \mathrm{~ms}$, no changes in the cyto-roGFP1 ratio were detected, ruling out mechanical artefacts.

D) The cyto-roGFP1 ratio clearly increased to local $\mathrm{H}_{2} \mathrm{O}_{2}(5 \mathrm{mM})$ puffs. Local redox challenge was achieved by applying repeated pressure puffs (100 ms, $5 \mathrm{~Hz}$ ) for $10 \mathrm{~s}$ (red mark), $30 \mathrm{~s}$ (blue mark) and $1 \mathrm{~min}$ (green mark). Displayed changes were quantified in the soma region. 
To optimize the parameters of local redox stimulation, dendrites of neurons were puffed by lower concentrated $\mathrm{H}_{2} \mathrm{O}_{2}(1 \mathrm{mM})$ in a single puff of $100 \mathrm{~ms}$. Again, the cyto-roGFP1 ratio increased, which was especially obvious in the soma (Figure $25 \mathrm{~A}$ ). To visualise cytosolic redox kinetics during redox challenge, a LOI along the neurons was drawn, starting from the lower dendrite through the soma, up to the upper dendrite (Figure $25 \mathrm{~B}$ ). Based on the time course of the cyto-roGFP1 ratiometric responses along this LOI, a kymograph was calculated to create a graphical representation of the evoked redox changes in the somatic and dendritic areas of a single neuron in time (Figure $25 \mathrm{C}$ ).

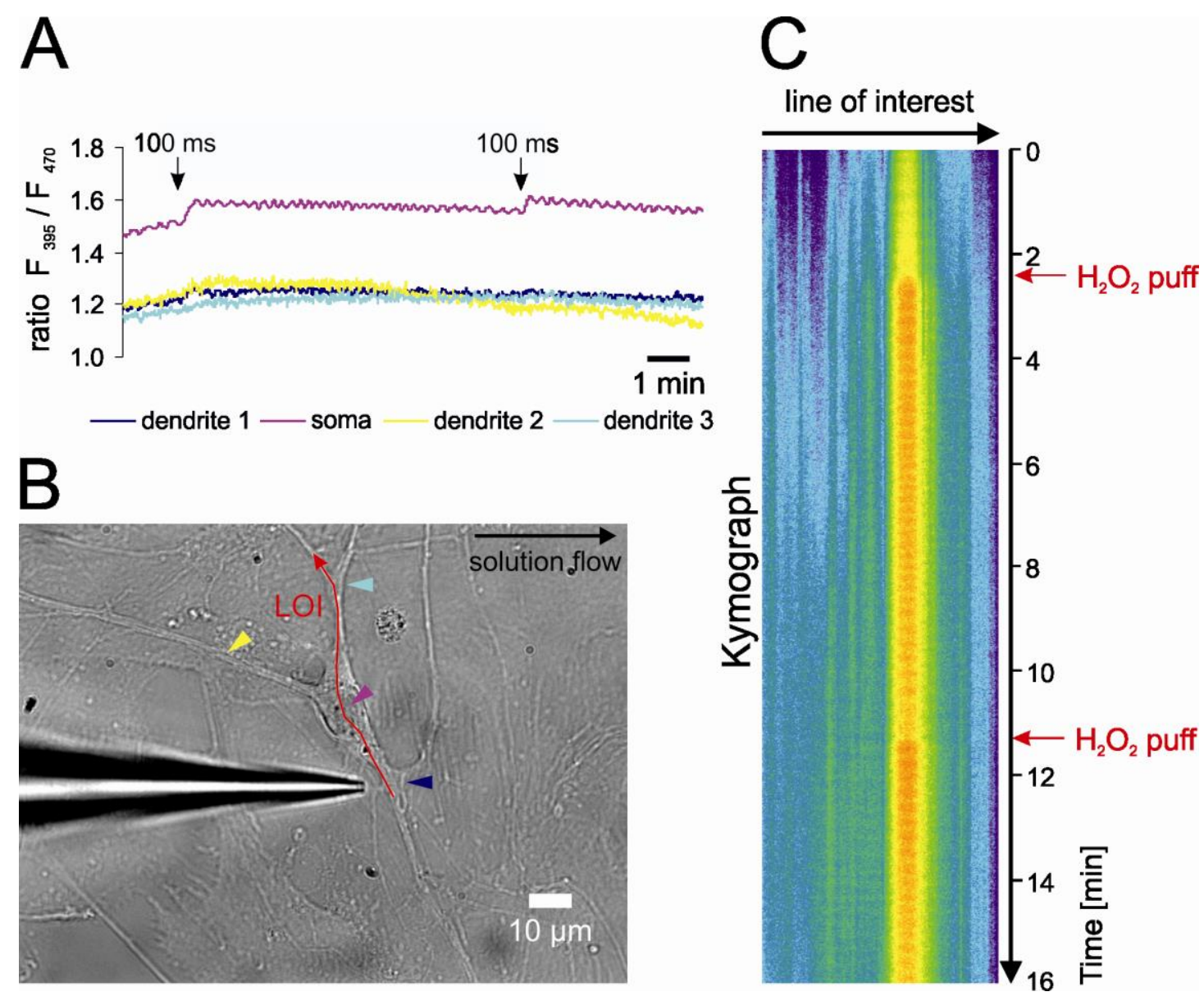

Figure 25. Sub-cellular changes within a single neuron upon local oxidant challenge.

A) The cyto-roGFP1 ratio revealed clear redox changes in the soma during $\mathrm{H}_{2} \mathrm{O}_{2}(1 \mathrm{mM})$ treatment.

B) To analyze the neuronal kinetics upon redox challenging induced by $\mathrm{H}_{2} \mathrm{O}_{2}$, and to calculate a kymograph, the LOI was drawn along the cell. Triangles indicate the particular areas of a neuron, whose redox changes are presented in panel $\mathrm{A}$. 
C) The kymograph summarizes the cyto-roGFP1 ratio changes within a single neuron in respect to the LOI from panel $B$ in time. The cyto-roGFP1 ratio increased especially in the cell body due to $\mathrm{H}_{2} \mathrm{O}_{2}$ application.

To prevent a potential spillover of high concentrated $\mathrm{H}_{2} \mathrm{O}_{2}(1 \mathrm{mM})$, even more subtle stimuli were performed by applying doses of $200 \mu \mathrm{M} \mathrm{H}_{2} \mathrm{O}_{2}$ in a single puff $(200 \mathrm{~ms})$. The cyto-roGFP1 ratio shifted towards more oxidizing conditions, which was especially pronounced in the soma (Figure 26 A). Similar to the previous experiment, a LOI was drawn along the neuron to obtain the sub-cellular kinetics during treatment (Figure $26 \mathrm{~B}$ ). Again, based on these data, a kymograph was created (Figure $26 \mathrm{C}$ ).

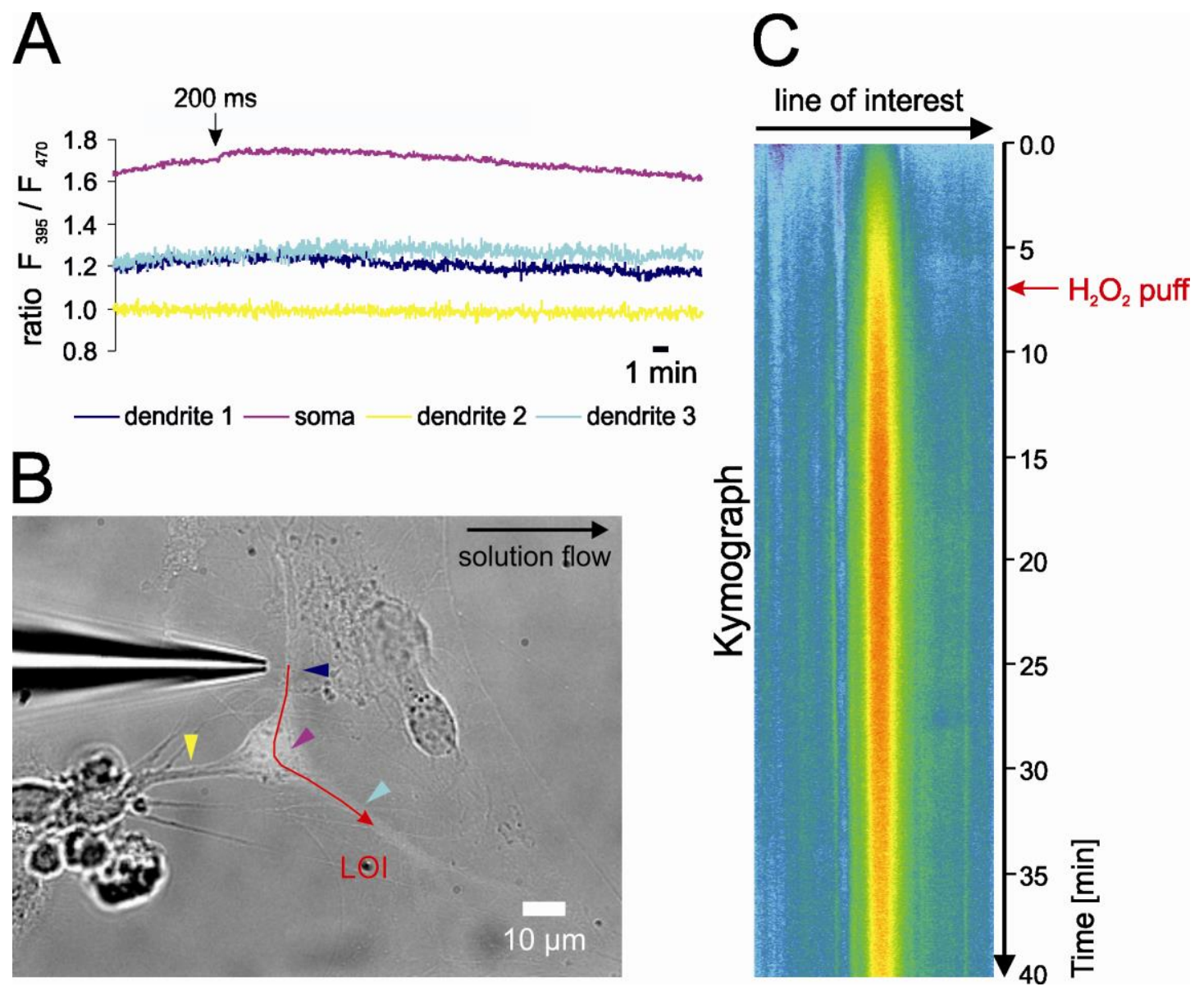

Figure 26. Local stimulation of a neuron with low doses of $\mathrm{H}_{2} \mathrm{O}_{2}$.

A) The cyto-roGFP1 ratio responded to redox modulation induced by $200 \mu \mathrm{M} \mathrm{H}_{2} \mathrm{O}_{2}$, which was applied as a local $200 \mathrm{~ms}$ pressure puff.

B) To calculate the kymograph and to analyze the neuronal kinetics, the LOI was drawn along the cell, starting from the upper dendrite. Triangles indicate the positions at which the displayed traces were extracted. 
C) The kymograph represents the temporal changes in redox balance within the displayed neuron. The $\mathrm{H}_{2} \mathrm{O}_{2}$ puff evoked an oxidation especially in the soma.

After confirming the feasibility of local puff application and the sensitivity of roGFP1 to even single puffs, sub-cellular changes in the cyto-roGFP1 ratio were now analyzed in more detail. For redox challenge of neurons, $\mathrm{H}_{2} \mathrm{O}_{2}(200$ $\mu \mathrm{M}$ ) was locally applied in a single puff mode for $200 \mathrm{~ms}$ in close proximity to a dendrite, and the resulting changes in sub-cellular redox balance were analyzed at different regions of interest to compare soma and dendrites.

As seen earlier (Figure 20), the cyto-roGFP1 pre-treatment baseline ratio was significantly $(p<0.001)$ higher in somata than in dendrites of WT and Mecp2-/y hippocampal neurons. Surprisingly, the cyto-roGFP1 pre-treatment baseline ratio was lower $(p<0.001)$ in $M e c p 2^{-1 y}$ dendrites as compared to WT dendrites (Figure $27 \mathrm{~A}$; Table 9).

However, the cyto-roGFP1 ratio shifted towards more oxidation in WT and $M_{e c p 2^{-1 y}}$ dendrites than in somata in response to local $\mathrm{H}_{2} \mathrm{O}_{2}$ application. This change was significant $(p<0.05)$ especially in WT neurons, but also evident as a solid trend in $\mathrm{Mecp2}^{-1 /}$ neurons (Figure $27 \mathrm{~B}$; Table 9). 


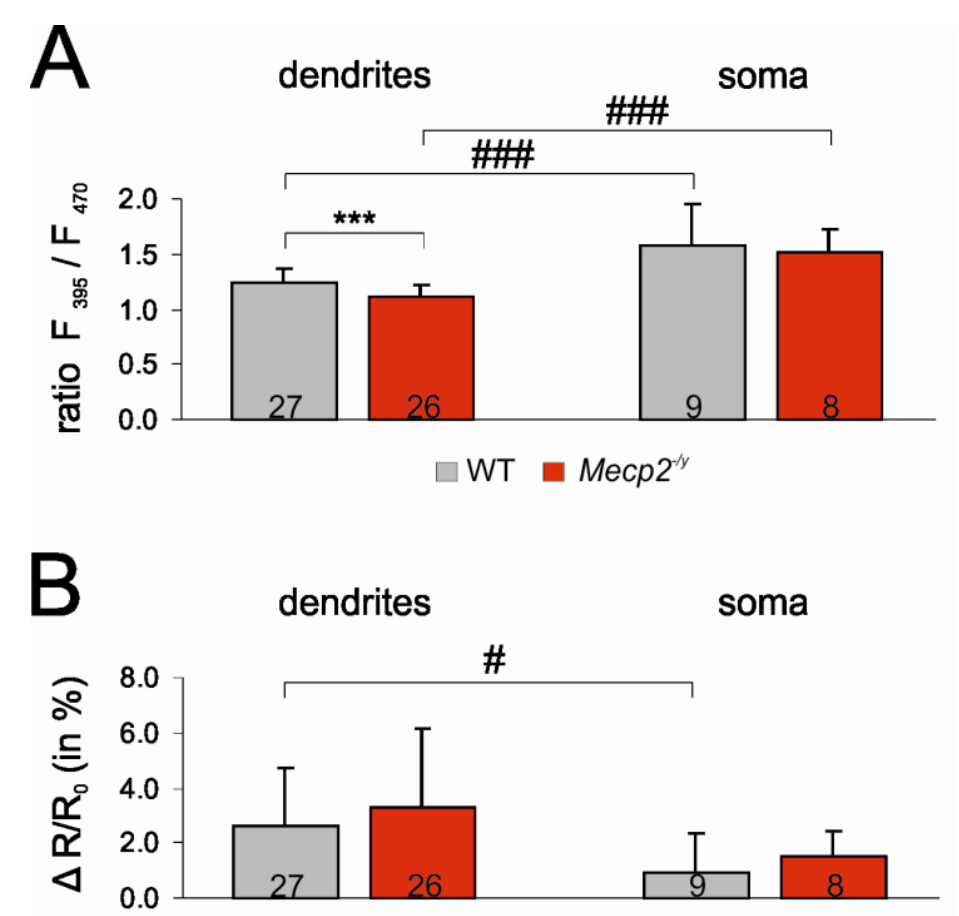

Figure 27. Relative redox changes in dendrites and soma of neurons.

A) Already under control conditions, the cyto-roGFP1 baseline ratio within the soma region was more $(\mathrm{p}<0.001)$ oxidized in comparison to dendrites. This was observed in both WT and Mecp2 $^{-/ y}$ hippocampal neurons (see also Figure 20).

B) Upon local puff stimulation of cultured neurons with $\mathrm{H}_{2} \mathrm{O}_{2}(200 \mu \mathrm{M}, 200 \mathrm{~ms})$, the cytoroGFP1 ratio was shifted to more oxidized levels in dendrites than in soma. This was especially obvious $(p<0.05)$ for WT neurons. 


\begin{tabular}{|c|c|c|c|c|c|}
\hline & \multirow{4}{*}{$\begin{array}{c}\text { WT } \\
1.3 \pm 0.1 \% \\
n_{\text {dendrites }}=27\end{array}$} & \multirow{4}{*}{$\begin{array}{c}\text { Mecp2 }^{-/ y} \\
1.1 \pm 0.1 \% \\
n_{\text {dendrites }}=26\end{array}$} \\
\hline & & & & & \\
\hline \multirow{4}{*}{ 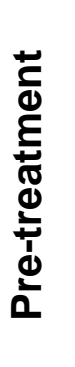 } & \multirow{4}{*}{\multicolumn{2}{|c|}{ 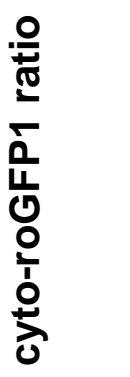 }} & & & \\
\hline & & & & & \\
\hline & & & Soma & $1.6 \pm 0.4 \%$ & $1.5 \pm 0.2 \%$ \\
\hline & & & & $n_{\text {somas }}=9$ & $n_{\text {somas }}=8$ \\
\hline \multirow{4}{*}{ 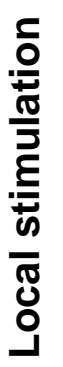 } & \multirow{4}{*}{\multicolumn{2}{|c|}{ 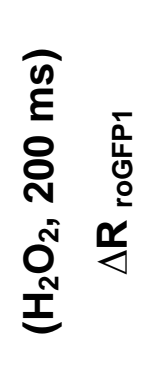 }} & Danduiton & $2.6 \pm 2.1 \%$ & $3.3 \pm 2.8 \%$ \\
\hline & & & & $n_{\text {dendrites }}=27$ & $n_{\text {dendrites }}=26$ \\
\hline & & & Soma & $0.9 \pm 1.4 \%$ & $1.5 \pm 0.9 \%$ \\
\hline & & & Juma & $n_{\text {somas }}=9$ & $n_{\text {somas }}=8$ \\
\hline
\end{tabular}

Table 9. Oxidative changes in dendrites and soma in response to locally stimulated neurons.

The soma was more oxidized in comparison to dendrites under control conditions in WT and $M e c p 2^{-/ y}$ hippocampal neurons. However, upon local neuronal stimulation with $\mathrm{H}_{2} \mathrm{O}_{2}(200 \mu \mathrm{M}$, $200 \mathrm{~ms}$ ), the cyto-roGFP1 ratio showed more intense oxidative responses in dendrites than in the soma.

\subsection{Electrical stimulation of neurons}

Since even defined sub-cellular redox changes could be detected in somatic and dendritic areas of cultured neurons, it was interesting to assess the local changes due to more physiological stimuli. Therefore, to evaluate the interplay of neuronal activity and redox balance, extracellular monopolar electrical stimulation was established.

For proof of principle, intact primary neuronal cultures were bulk loaded with 5 $\mu \mathrm{M}$ of calcium $\left(\mathrm{Ca}^{2+}\right)$ dye Fluo-3 in sterile and humidified $\left(37^{\circ} \mathrm{C}, 5 \% \mathrm{CO}_{2}\right)$ conditions for 30 min (Figure $28 \mathrm{~A}$ ). The cultures were next placed into the submersion style recording chamber, and an extracellular stimulation electrode was positioned close to a dendrite (Figure $28 \mathrm{~B}$ ). For electrical stimulation, $100 \mu \mathrm{A}$ current, $1 \mathrm{~ms}$ pulses, $5 \mathrm{~s}$ train duration and frequencies of $2 \mathrm{~Hz}$ and $5 \mathrm{~Hz}$ were applied. In response to such stimuli, Fluo-3 fluorescence 
markedly increased, confirming successful activation of this neuron. However, after a second stimulation, cell rupture was frequently observed, which was indicated by a marked and rapid drop of Fluo-3 fluorescence (Figure $28 \mathrm{C}$ ).
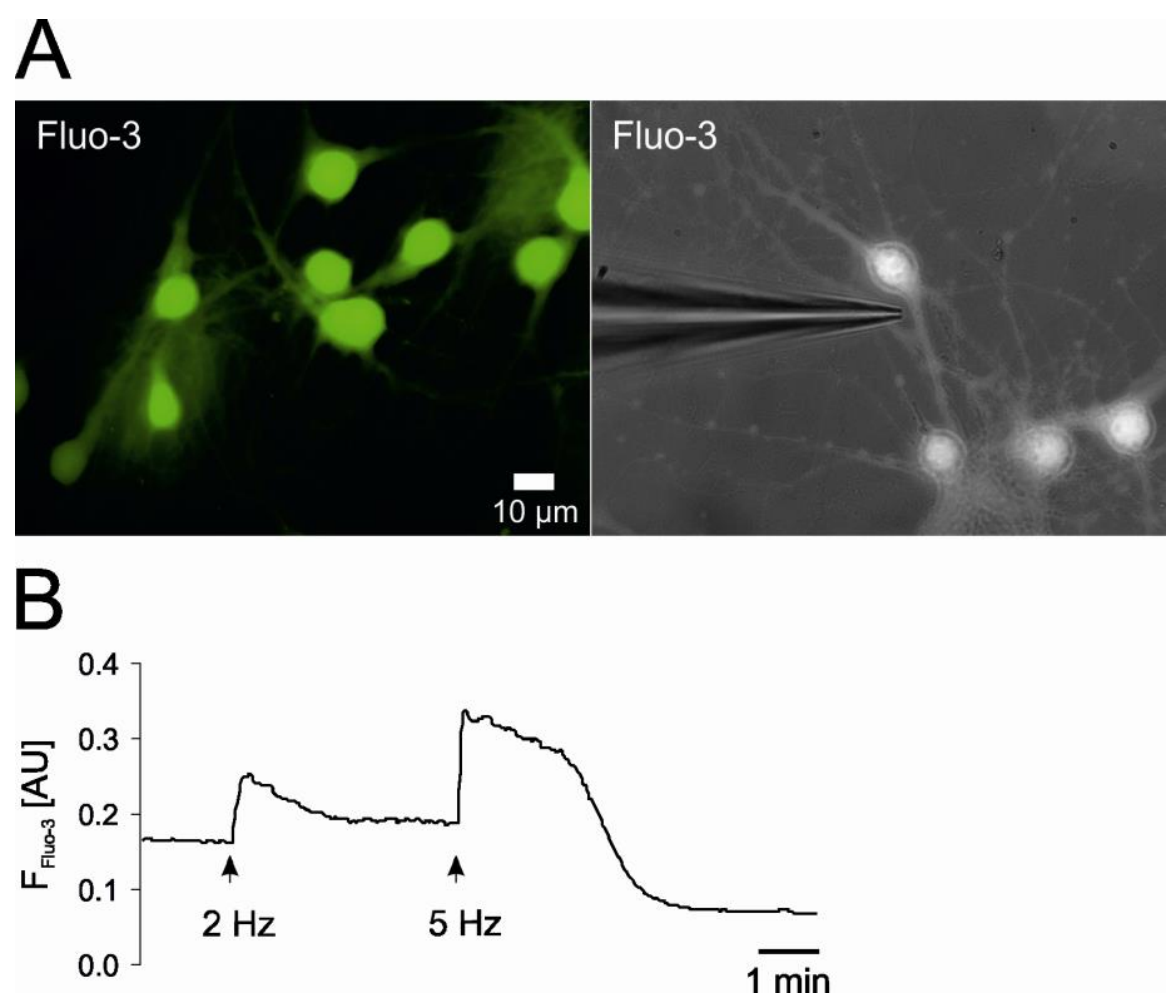

Figure 28. Principle of electrical stimulation.

A) Primary hippocampal neurons were stained with the calcium-sensitive dye Fluo-3. The extracellular recording electrode was positioned closely to a neuronal dendrite. The scale bar applies to both images.

B) Fluorescence responses were evoked by $100 \mu \mathrm{A}$ of current, $1 \mathrm{~ms}$ pulse, $5 \mathrm{~s}$ train duration, and $2 \mathrm{~Hz}$ and $5 \mathrm{~Hz}$ frequency. Note that the Fluo-3 fluorescence increased immediately upon electrical stimulation.

In order to quantify the Fluo-3 responses upon massive neuronal depolarisation and the related strong $\mathrm{Ca}^{2+}$ influx, neurons were depolarised by ACSF containing $40 \mathrm{mM}$ potassium $\left(\mathrm{K}^{+}\right)$. To prevent any osmotic stress, ACSF with only $90 \mathrm{mM}$ sodium $\left(\mathrm{Na}^{+}\right)$was used in these tests. The Fluo-3 fluorescence increased by a total of $505.4 \pm 145.4 \%(n=16)$, and it fully recovered after the treatment (Figure 29). 

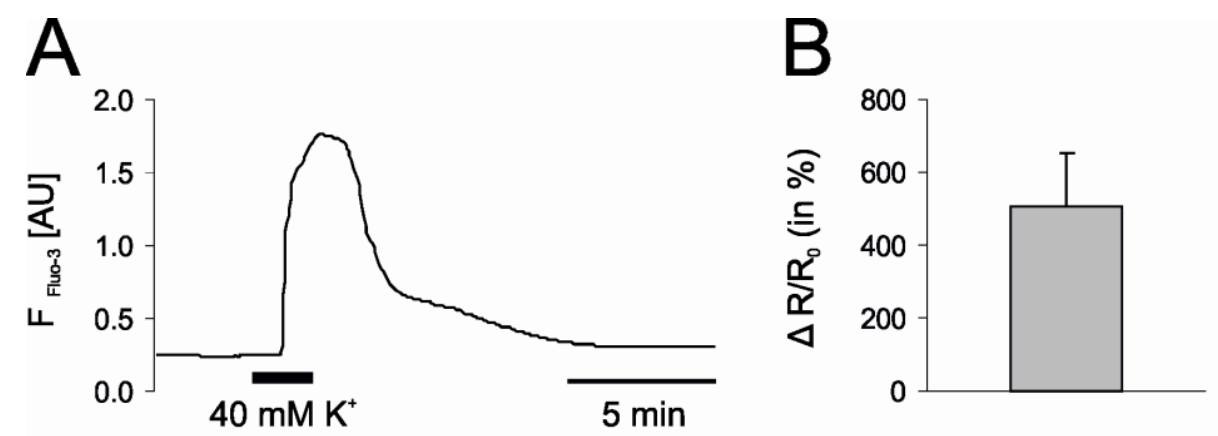

Figure 29. Fluo-3 responses upon massive neuronal depolarisation.

A) Depolarisation $\left(40 \mathrm{mM} \mathrm{K}^{+}\right)$of neurons induced pronounced transient $\mathrm{Ca}^{2+}$ influx indicated by a marked increase in Fluo-3 fluorescence.

B) On average, $\mathrm{K}^{+}$-mediated depolarisation caused an increase of Fluo-3 fluorescence by $505.4 \pm 145.4 \%(n=16)$.

After confirming that electrical stimulation does in principle work and elicits a much more moderate $\mathrm{Ca}^{2+}$ influx than general cellular depolarisation, this approach was next applied to the cyto-roGFP1-transduced hippocampal primary neurons. Parameters of $100 \mu \mathrm{A}$ current, $1 \mathrm{~ms}$ pulse, $5 \mathrm{~s}$ duration and frequencies of $2 \mathrm{~Hz}$ and $5 \mathrm{~Hz}$ were applied. Against expectation, after a first electrical stimulation, the cyto-roGFP1 ratio fluorescence decreased and a second electrical stimulation induced neuronal cell loss (Figure 30).

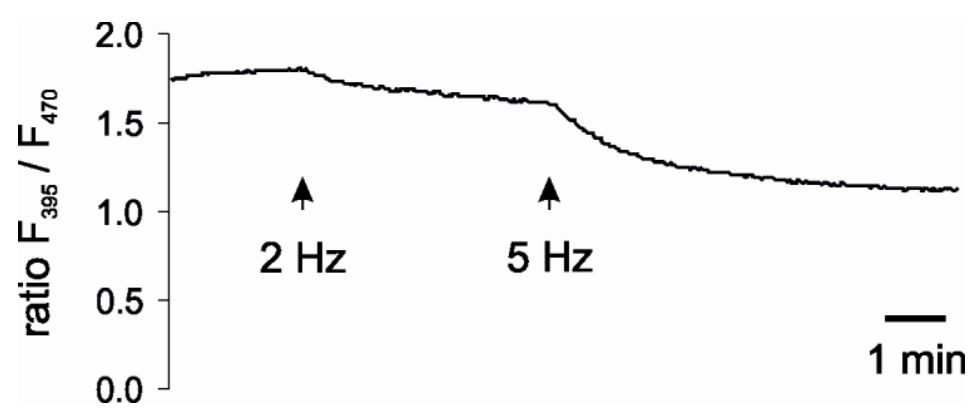

Figure 30. The cyto-roGFP1 ratiometric response to extracellular electrical stimulation.

Upon electrical stimulation (100 $\mu \mathrm{A}$ current, $1 \mathrm{~ms}$ pulse, $5 \mathrm{~s}$ duration, $1 \mathrm{~Hz}$ and $2 \mathrm{~Hz}$ frequency), the cyto-roGFP1 ratio fluorescence decreased. A second electrical stimulation induced a marked fluorescence drop, suggesting massively impaired neuronal integrity.

In an attempt to maintain cellular viability, stimulation intensities were reduced. Indeed, the contrary neuronal responses could now be observed, at least in some trials. In some neurons, the cyto-roGFP1 ratio increased upon 
electrical stimulation when $20 \mu \mathrm{A}$ current in a single $10 \mathrm{~ms}$ pulse was applied (Figure $31 \mathrm{~A}$ ). In others, the cyto-roGFP1 ratio markedly decreased right after the stimulation (Figure $31 \mathrm{~B}$ ). Stimulation of neuronal cultures with even more gentle parameters (10 $\mu \mathrm{A}$ current, $1 \mathrm{~ms}$ pulse) still induced quite variable responses. The cyto-roGFP1 ratio shifted towards more oxidizing conditions in some cells (Figure $31 \mathrm{C}$ ), whereas in others it rapidly decreased upon electrical stimulation (Figure 31 D). Therefore, one might speculate that roGFP1-transduced neurons are more fragile to electrical stimulation than $\mathrm{Ca}^{2+}$ loaded neurons, which tolerated the procedure.
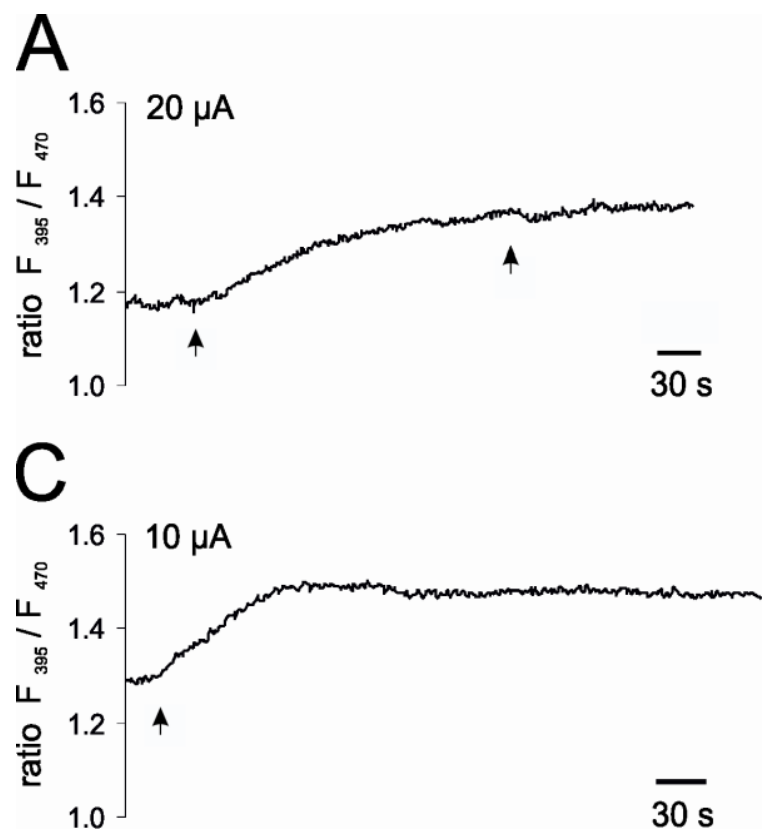
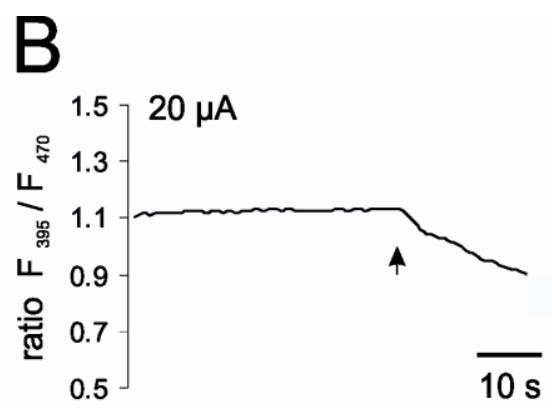

$D$

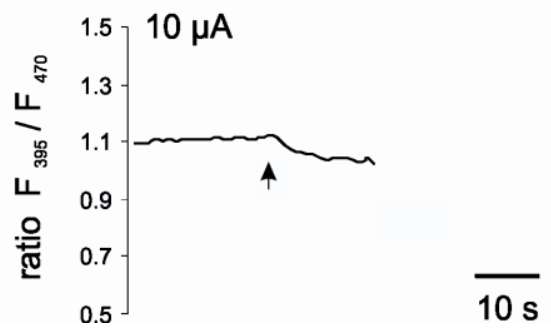

Figure 31. Variability of cyto-roGFP1 ratio responses to extracellular electrical stimulation.

A) Electrical stimulation of neurons (20 $\mu \mathrm{A}, 10 \mathrm{~ms}$ pulse) resulted in an increase of the cytoroGFP1 ratio, indicating oxidation. A second stimulation did not noticeably affect the cytoroGFP1 ratio.

B) Stimulation of another neuronal cell induced a rapid decrease in the cyto-roGFP1 ratio and cell loss.

C) Stimulation of a neuron with a more gentle current (10 $\mu \mathrm{A}, 1 \mathrm{~ms}$ pulse) increased the cytoroGFP1 ratio, which then reached a plateau stage.

D) Yet, even gentle electrical stimulation of neurons sometimes resulted in cell loss. 


\subsection{The effect of neurotransmitters on cellular redox balance}

In order to compare the redox responses of WT and Mecp2 $2^{-/ y}$ hippocampal neurons, evoked by various neurotransmitters, glutamate, serotonin, norepinephrine and dopamine were applied to cyto-roGFP1-transduced primary cultured neurons.

Glutamate (50 $\mu \mathrm{M}, 5 \mathrm{~min})$ caused biphasic responses, consisting of an initial decrease followed by a marked increase of roGFP1 ratio. Since glutamate induced continuously rising responses of the cyto-roGFP1 ratio, which did not recover upon wash-out, the redox-level reached after 30 min was taken for statistical calculations. Genotypic comparison revealed that the initial drop was more $(p<0.001)$ intense in WT than in $M e c p 2^{-/ y}$ neurons. The secondary increase was more pronounced $(p<0.001)$ in $M e c p 2^{-/ y}$ neurons in comparison to WT (Figure $32 \mathrm{~A}$, F; Table 10).

The cyto-roGFP1 ratio increased also more intensively $(p<0.001)$ in $M e c p 2^{-/ y}$ neurons when norepinephrine $(200 \mu \mathrm{M}, 3 \mathrm{~min})$ was applied as compared to WT (Figure 32 B, F; Table 10). Moreover, after only partial recovery upon wash-out of norepinephrine, the cyto-roGFP1 ratio continued to rise. Similar, but even stronger effects occurred in $M c{ }^{-1 / y}$ neurons exposed to a higher dose of norepinephrine $(500 \mu \mathrm{M}, 3 \mathrm{~min})$ (Figure $32 \mathrm{C}, \mathrm{F}$; Table 10). An increase of the cyto-roGFP1 ratio was also induced by serotonin $(100 \mu \mathrm{M}, 10$ $\mathrm{min})$, which was again more pronounced $(\mathrm{p}<0.05)$ in $M e c p 2^{-/ y}$ than in WT neurons (Figure $32 \mathrm{D}$, F; Table 10). Furthermore, in contrast to WT, the cytoroGFP1 ratio of $M e c p 2^{-/ y}$ cells did not recover to its pre-treatment baseline after serotonin treatment. Application of dopamine ( $500 \mu \mathrm{M}, 3 \mathrm{~min})$ shifted the roGFP1 ratio towards more oxidizing conditions, which was also intensified $(\mathrm{p}<0.05)$ in $\mathrm{Mecp2}^{-1 /}$ neurons (Figure $32 \mathrm{E}, \mathrm{F}$; Table 10). In contrast to the other tested neurotransmitters, the redox changes evoked by dopamine readily recovered in both genotypes after wash out. Nevertheless, the oxidative shift reduced, was again more intense in $M e c p 2^{-1 / y}$ neurons. 
A
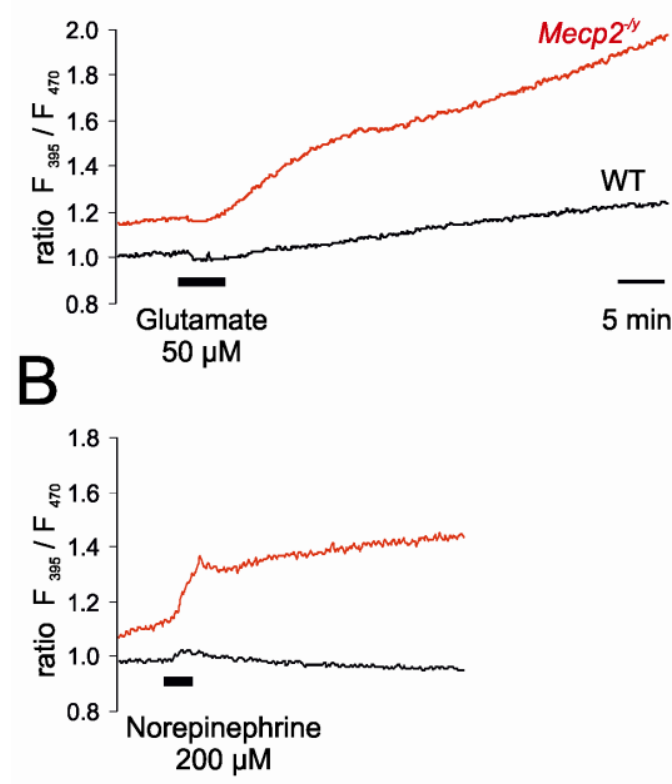

C

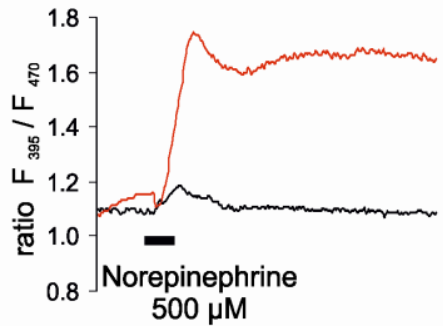

$\mathrm{D}$

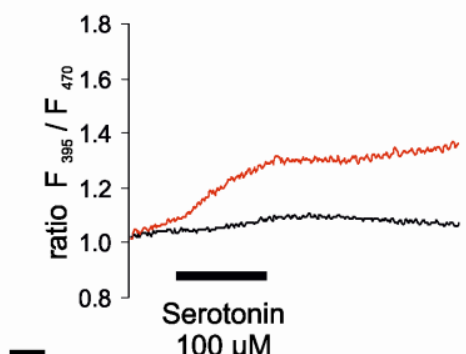

E
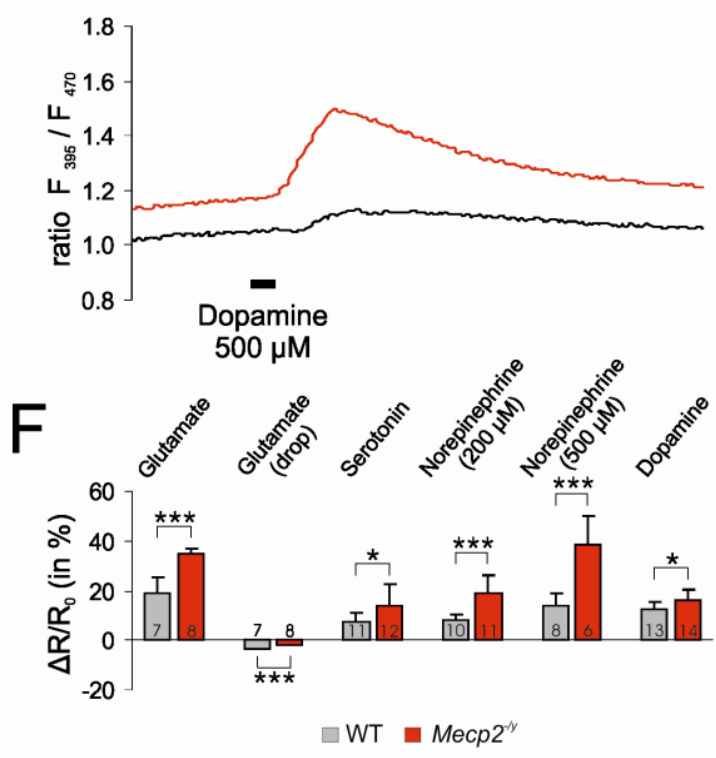

\section{Figure 32. The cyto-roGFP1 ratio responses to neurotransmitters.}

A) Under treatment with glutamate, the cyto-roGFP1 ratio shifted towards more $(p<0.001)$ oxidizing conditions in $M e c p 2^{-/ y}$ neurons as compared to WT. Also, application of glutamate caused an initial drop in the cyto-roGFP1 ratio, which was markedly less $(p<0.001)$ intense in $M_{\text {ecp2 }} 2^{-y}$ neurons than in WT. Time scaling is identical for all of the traces shown.

B and C) Smaller $(200 \mu \mathrm{M})$ and higher $(500 \mu \mathrm{M})$ doses of norepinephrine resulted in an increase in cyto-roGFP1 ratio, which was more pronounced $(p<0.001)$ in $M e c p 2^{-1 y}$ neurons.

D) Treatment with serotonin elicited a shift towards oxidation, which was intensified $(p<0.001)$ in $\mathrm{Mecp}^{-/ y}$ neurons.

E) Also, dopamine induced a more intense $(\mathrm{p}<0.05)$ oxidation in $M e c p 2^{-/ y}$ neurons.

F) Statistical overview of the quantified cyto-roGFP1 responses to neurotransmitters in WT and $M e c p 2^{-/ y}$ neurons. 


\begin{tabular}{|c|c|c|}
\hline \multirow[b]{2}{*}{ Neurotransmitter } & \multicolumn{2}{|c|}{$\begin{array}{l}\text { Evoked changes in roGFP1 ratio } \\
\qquad\left(\mathbf{F}_{395} / \mathbf{F}_{470}\right), \Delta \mathbf{R}_{\text {roGFP1 }}\end{array}$} \\
\hline & WT & Mecp2 $^{-/ y}$ \\
\hline Serotonin & $\begin{array}{c}7.5 \pm 3.6 \% \\
n=11\end{array}$ & $\begin{array}{c}14.0 \pm 8.8 \% \\
n=12\end{array}$ \\
\hline $\begin{array}{l}\text { Norepinephrine } \\
(200 \mu \mathrm{M})\end{array}$ & $\begin{array}{c}7.9 \pm 2.5 \% \\
n=10\end{array}$ & $\begin{array}{c}18.9 \pm 7.2 \% \\
n=11\end{array}$ \\
\hline $\begin{array}{c}\text { Norepinephrine } \\
(500 \mu \mathrm{M})\end{array}$ & $\begin{array}{c}14.2 \pm 4.7 \% \\
n=8\end{array}$ & $\begin{array}{c}38.5 \pm 11.8 \% \\
n=6\end{array}$ \\
\hline $\begin{array}{l}\text { Glutamate } \\
\text { (initial drop) }\end{array}$ & $\begin{array}{c}-3.3 \pm 0.5 \% \\
n=7\end{array}$ & $\begin{array}{c}-1.7 \pm 0.5 \% \\
n=8\end{array}$ \\
\hline $\begin{array}{c}\text { Glutamate } \\
\text { (secondary increase) }\end{array}$ & $\begin{array}{c}18.8 \pm 6.4 \% \\
n=7\end{array}$ & $\begin{array}{c}34.6 \pm 2.2 \% \\
n=8\end{array}$ \\
\hline Dopamine & $\begin{array}{c}12.3 \pm 3.0 \% \\
n=13\end{array}$ & $\begin{array}{c}15.7 \pm 5.0 \% \\
n=14\end{array}$ \\
\hline
\end{tabular}

Table 10. Effects of neurotransmitters on the cyto-roGFP1 ratio in hippocampal cultured neurons.

\subsection{Mechanisms of transmitter-mediated redox changes}

To identify the very molecular mechanisms, which play a role in neuronal redox events in response to neurotransmitters, potential candidate processes such as $\mathrm{Ca}^{2+}$ influx, mitochondrial respiration, NADPH oxidase (NOX) and xanthine oxidase ( $\mathrm{XO}$ ) activation as well as altered cyclic adenosine monophosphate (cAMP) levels were evaluated. In these experiments, the neuronal responses to glutamate $(50 \mu \mathrm{M}, 5 \mathrm{~min})$ and dopamine (500 $\mu \mathrm{M}, 3$ $\min$ ) were assessed. These two transmitters were chosen, since glutamate evoked the most intensified and continuously rising responses of the cytoroGFP1 ratio, which did not recover upon wash-out, whereas dopamine responses always fully recovered in both genotypes.

Treatment of neurons with glutamate under $\mathrm{Ca}^{2+}$ free conditions still caused a marked $(p<0.001)$ shift of the cyto-roGFP1 ratio towards oxidation in $M e c p 2^{-/ y}$ 
neurons, whereas the oxidizing redox response in WT neurons was almost completely abolished (Figure 33 A, E; Table 11). Interestingly, the initial drop upon glutamate application was now significantly $(\mathrm{p}<0.05)$ larger in $M e c p 2^{-/ y}$ than in WT neurons (Figure $33 \mathrm{~F}$; Table 11). A continuous shift towards oxidation was still induced by glutamate in WT and $M e c p 2^{-/ y}$ neurons when mitochondria were uncoupled by FCCP (1 $1 \mathrm{M}, 8 \mathrm{~min}$ ) (Figure $33 \mathrm{~B}$, E; Table 11). Yet, upon inhibition of NOX by DPI (20 $\mu \mathrm{M}, 8 \mathrm{~min})$ the glutamatemediated redox responses were almost abolished. Only a tiny shift towards oxidation remained (Figure $33 \mathrm{C}$, E; Table 11). The responses to glutamate were also suppressed when XO was blocked by allopurinol (200 $\mu \mathrm{M}, 10 \mathrm{~min})$; instead, a very small reducing shift could now be observed (Figure 33 D, E; Table 11). 

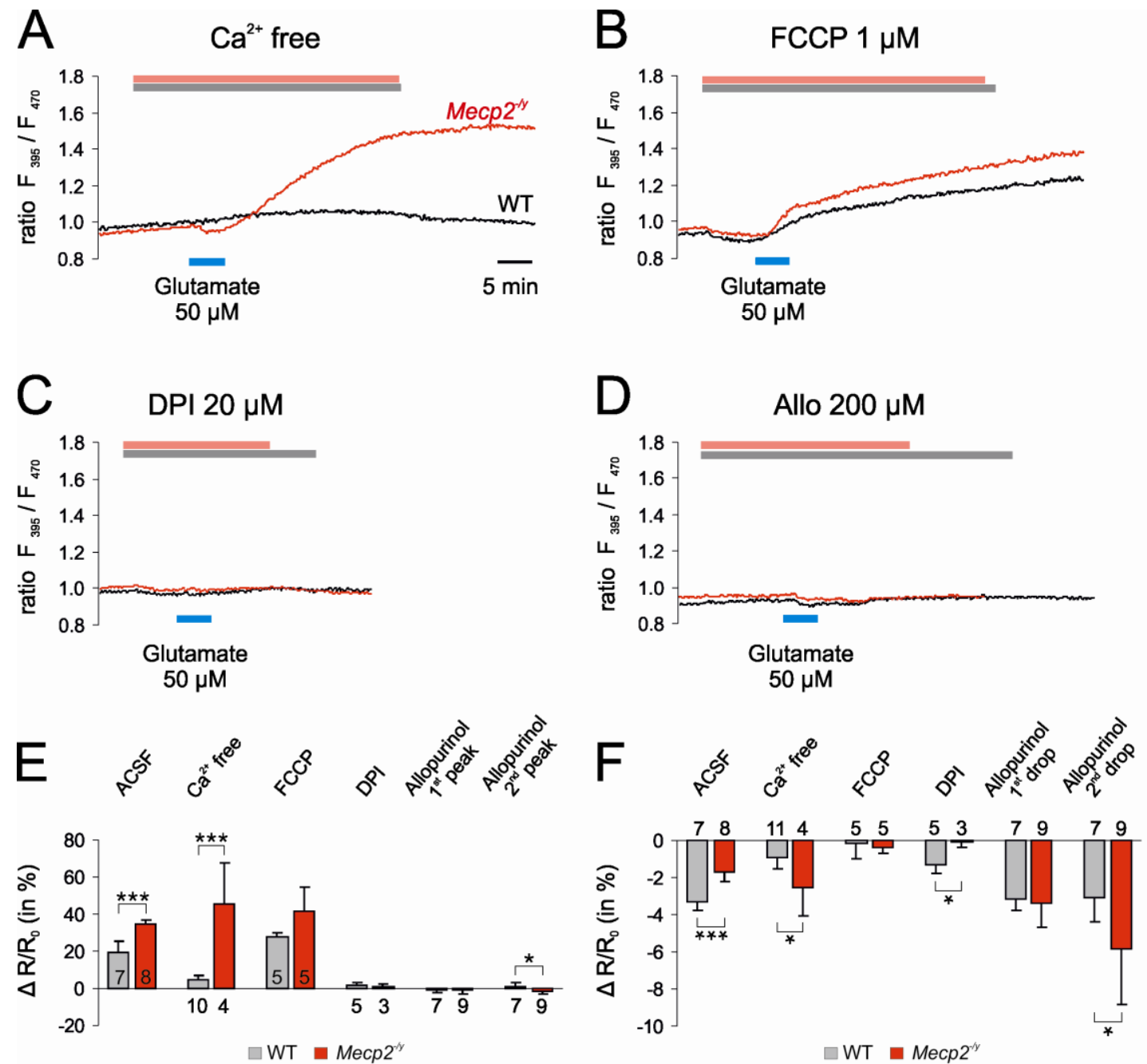

Figure 33. Mechanistic deciphering of the glutamate-mediated roGFP1 responses.

A) Glutamate caused an increase in the roGFP1 ratio under free $\mathrm{Ca}^{2+}$ conditions, which was especially obvious $(p<0.001)$ for $M e c p 2^{-y}$ neurons. In WT neurons, the redox responses to glutamate were markedly dampened. The scale bar is equal for all traces displayed.

B) After mitochondrial uncoupling, the roGFP1 baseline slightly decreased, but still glutamate induced a clear oxidizing response of the roGFP1 ratio in WT and $M e c p 2^{-1 y}$ neurons. Also, the cyto-roGFP1 ratio showed a solid tendency towards more intense oxidation in Mecp2 $2^{-y}$ neurons as compared to WT.

C) Blocking of NOX by DPI almost completely abolished the roGFP1 response to glutamate in WT and $M_{\text {ecp }}{ }^{-/ y}$ neurons.

D) Inhibition of XO by allopurinol fully blocked the responses of the roGFP1 ratio to glutamate in WT and $M е с p 2^{-/ y}$ neurons. Note that the roGFP1 ratio now even decreased slightly.

E) Statistical overview of the quantified responses of WT and $M e c p 2^{-/ y}$ neurons to glutamate under $\mathrm{Ca}^{2+}$ free conditions, mitochondrial uncoupling and inhibition of NOX and XO. The bar plot of the control conditions under ACSF were included again as a reference of the original response magnitude. 
F) Summary of the initial reducing shifts under the respective physiological conditions. The bar plot of the control conditions under ACSF were included for comparison.

Application of dopamine still induced a marked shift of the cyto-roGFP1 ratio towards oxidation in WT and Mecp2-/y neurons under $\mathrm{Ca}^{2+}$ free conditions (Figure 34 A, D; Table 11). Also, an increase of the cyto-roGFP1 ratio occurred when glutamate was applied upon mitochondrial uncoupling (Figure 34 B, D; Table 11). Surprisingly, under these conditions, this shift of the cytoroGFP1 ratio towards oxidation was now more $(p<0.05)$ intense in WT than in Mecp2 ${ }^{-1 y}$ neurons. Supplementation of neurons with dopamine upon NOX blockade evoked only a very slight residual shift of the cyto-roGFP1 ratio towards oxidation in WT and Mecp2 $2^{-/ y}$ neurons (Figure $34 \mathrm{C}, \mathrm{D}$; Table 11).
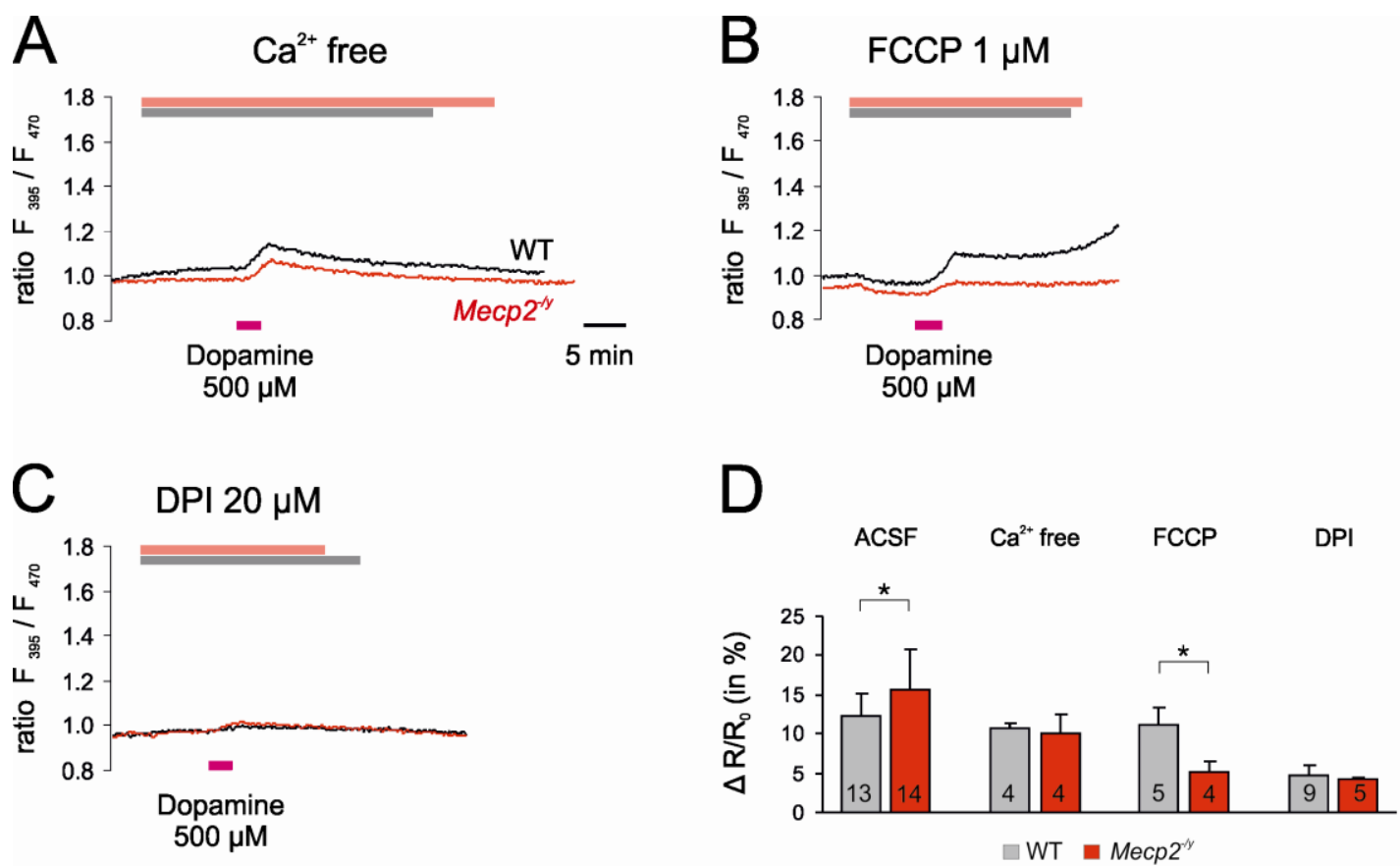

Figure 34. Mechanistic analysis of dopamine-mediated redox responses.

A) Administration of dopamine evoked a shift of the cyto-roGFP1 ratio towards oxidation under $\mathrm{Ca}^{2+}$ free conditions in WT and $M e c p 2^{-/ y}$ neurons. The time scaling is identical for all traces displayed.

B) Application of dopamine during mitochondrial uncoupling by FCCP still induced a clear shift of the cyto-roGFP1 ratio towards more oxidizing conditions in WT neurons, which was, however, less intense in $M e c p 2^{-/ y}$ neurons.

C) Inhibition of NOX by DPI almost completely abolished the dopamine reduced oxidative shifts in both genotypes. 
D) Statistical overview of the quantified responses of WT and $M e c p 2^{-1 y}$ neurons to dopamine upon $\mathrm{Ca}^{2+}$ withdrawal, mitochondrial uncoupling and inhibition of NOX. Dopamine responses under control conditions (ACSF) are shown as a reference. 


\begin{tabular}{|c|c|c|c|}
\hline \multirow[b]{2}{*}{ Treatment } & \multirow[b]{2}{*}{ Neurotransmitter } & \multicolumn{2}{|c|}{$\begin{array}{c}\text { Evoked changes in roGFP1 ratio } \\
\left(\mathbf{F}_{395} / \mathbf{F}_{470}\right), \Delta \mathbf{R}_{\text {roGFP1 }}\end{array}$} \\
\hline & & WT & Mecp2 $^{-/ y}$ \\
\hline \multirow{3}{*}{$\mathrm{Ca}^{2+}$ free } & $\begin{array}{l}\text { Glutamate } \\
\text { (initial drop) }\end{array}$ & $\begin{array}{c}-0.9 \pm 0.6 \% \\
n=11\end{array}$ & $\begin{array}{c}-2.5 \pm 1.6 \% \\
n=4\end{array}$ \\
\hline & $\begin{array}{l}\text { Glutamate } \\
\text { (secondary } \\
\text { response) }\end{array}$ & $\begin{array}{c}4.5 \pm 2.6 \% \\
n=10\end{array}$ & $\begin{array}{c}45.4 \pm 22.5 \% \\
n=4\end{array}$ \\
\hline & DA & $\begin{array}{c}10.7 \pm 0.7 \% \\
n=4\end{array}$ & $\begin{array}{c}10.0 \pm 2.5 \% \\
n=4\end{array}$ \\
\hline \multirow{3}{*}{ FCCP } & $\begin{array}{l}\text { Glutamate } \\
\text { (initial drop) }\end{array}$ & $\begin{array}{c}-0.2 \pm 0.8 \% \\
n=5\end{array}$ & $\begin{array}{c}-0.4 \pm 0.3 \% \\
n=5\end{array}$ \\
\hline & $\begin{array}{l}\text { Glutamate } \\
\text { (secondary } \\
\text { response) }\end{array}$ & $\begin{array}{c}27.7 \pm 2.7 \% \\
n=5\end{array}$ & $\begin{array}{c}41.4 \pm 13.3 \% \\
n=5\end{array}$ \\
\hline & DA & $\begin{array}{c}11.3 \pm 2.1 \% \\
n=5\end{array}$ & $\begin{array}{c}5.1 \pm 1.3 \% \\
n=4\end{array}$ \\
\hline \multirow{3}{*}{ DPI } & $\begin{array}{l}\text { Glutamate } \\
\text { (initial drop) }\end{array}$ & $\begin{array}{c}-1.3 \pm 0.5 \% \\
n=5\end{array}$ & $\begin{array}{c}-0.1 \pm 0.3 \% \\
n=4\end{array}$ \\
\hline & $\begin{array}{l}\text { Glutamate } \\
\text { (secondary } \\
\text { response) }\end{array}$ & $\begin{array}{c}1.6 \pm 1.1 \% \\
n=5\end{array}$ & $\begin{array}{c}1.1 \pm 1.0 \% \\
n=3\end{array}$ \\
\hline & DA & $\begin{array}{c}4.8 \pm 1.3 \% \\
n=9\end{array}$ & $\begin{array}{c}4.2 \pm 0.3 \% \\
n=5\end{array}$ \\
\hline \multirow{4}{*}{ Allopurinol } & $\begin{array}{l}\text { Glutamate } \\
\left(1^{\text {st }} \text { peak }\right)\end{array}$ & $\begin{array}{c}-0.7 \pm 1.6 \% \\
n=7\end{array}$ & $\begin{array}{c}-0.9 \pm 2.0 \% \\
n=9\end{array}$ \\
\hline & $\begin{array}{l}\text { Glutamate } \\
\left(2^{\text {nd }} \text { peak }\right)\end{array}$ & $\begin{array}{c}0.8 \pm 2.0 \% \\
n=7\end{array}$ & $\begin{array}{c}-1.7 \pm 1.5 \% \\
n=9\end{array}$ \\
\hline & $\begin{array}{l}\text { Glutamate } \\
\left(1^{\text {st }} \text { drop) }\right.\end{array}$ & $\begin{array}{c}-3.2 \pm 0.6 \% \\
n=7\end{array}$ & $\begin{array}{c}-3.4 \pm 1.3 \% \\
n=9\end{array}$ \\
\hline & $\begin{array}{l}\text { Glutamate } \\
\left(2^{\text {nd }} \text { drop }\right)\end{array}$ & $\begin{array}{c}-3.1 \pm 1.3 \% \\
n=7\end{array}$ & $\begin{array}{c}-5.8 \pm 3.0 \% \\
n=9\end{array}$ \\
\hline
\end{tabular}

Table 11. Redox changes in response to glutamate and dopamine upon $\mathrm{Ca}^{2+}$ depletion, mitochondrial uncoupling, NADPH oxidase inhibition and xanthine oxidation blockade. 
To assess briefly, whether G-protein and CAMP mediated signalling is involved in redox changes, the cyto-roGFP1 transduced neurons were treated with forskolin $(10 \mu \mathrm{M}, 10 \mathrm{~min})$ in order to activate G-proteins and hence, adenylate cyclase. Promotion of the cAMP pathway did, however, not contribute to redox changes under control conditions, since the cyto-roGFP1 ratio only barely increased by $3.6 \pm 1.4 \%(n=6)$.

To assess a particular contribution of the respective ROS generating candidate mechanisms to steady state baseline redox conditions, the cytoroGFP1 baseline ratios under $\mathrm{Ca}^{2+}$ free conditions and mitochondrial uncoupling as well as NADPH and xanthine oxidases inhibition were calculated and compared with the control cyto-roGFP1 baseline ratios. The largest change in cyto-roGFP1 ratio was observed under mitochondria uncoupling as it decreased by $0.4 \%$ in WT and $\mathrm{Mecp2}^{-/ y}$ neurons (Table 12). 


\begin{tabular}{|c|c|c|}
\hline \multicolumn{3}{|c|}{ Evoked changes in roGFP1 ratio $\left(F_{395} / F_{470}\right)$} \\
\hline WT & \multirow{2}{*}{$\mathrm{Ca}^{2+}$ free } & $1.02 \pm 0.02 \%$ \\
\hline $\operatorname{Mecp2}^{-1 / y}$ & & $\begin{array}{c}1.03 \pm 0.03 \% \\
n=8\end{array}$ \\
\hline WT & \multirow{2}{*}{ FCCP } & $\begin{array}{c}0.96 \pm 0.02 \% \\
n=11\end{array}$ \\
\hline Mecp2 $2^{-y}$ & & $\begin{array}{c}0.96 \pm 0.01 \% \\
n=9\end{array}$ \\
\hline WT & \multirow{2}{*}{ DPI } & $\begin{array}{c}0.99 \pm 0.01 \% \\
n=14\end{array}$ \\
\hline Mecp2 $^{-/ y}$ & & $\begin{array}{c}1.01 \pm 0.02 \% \\
n=11\end{array}$ \\
\hline WT & \multirow{2}{*}{ Allopurinol } & $\begin{array}{c}1.02 \pm 0.01 \% \\
n=7\end{array}$ \\
\hline $\operatorname{Mecp2}^{-/ y}$ & & $\begin{array}{c}1.01 \pm 0.00 \% \\
n=9\end{array}$ \\
\hline
\end{tabular}

Table 12. RoGFP1 baseline changes upon arrest of potential cellular ROS sources.

Listed are changes of the cytosolic roGFP1 baseline ratios of WT and Mecp2 ${ }^{-/ y}$ neurons in various redox conditions in respect to the control roGFP1 baseline ratio under ACSF.

\subsection{The roGFP1-transgenic mice as a novel tool for cellular redox imaging during disease progression.}

To overcome the limitations of cultured preparations and surgery interventions, and to allow for the more complex cellular redox imaging especially at older stages, our group has recently generated transgenic mice, carrying the neuronal specific mitochondrial roGFP1 (roGFP1m) and cytosolic roGFP1 (roGFP1c) sensors, respectively (Wagener et al. 2016).

To assess any potential redox alterations in $M e c p 2^{-1 y}$ neurons of adult symptomatic Rett mice, and to correlate the Rett syndrome disease 
progression with impaired redox balance, the transgenic roGFP1c males were cross-bred with $\mathrm{Mecp}^{-/+}$females. The very first obtained two roGFP1c $M_{\text {ecp2 }}{ }^{-/ y}$ mice were here tested for redox changes specifically in neurons. Acute brain slices from adult (PD 47-52) mice were prepared. Neurons of the hippocampal regions CA1, CA3 and dentate gyrus (DG) as well as cortical neurons were imaged and the cytosolic roGFP1 baseline ratio was calculated by using the recently developed dual laser based excitation ratiometric 2photon imaging (Figure 35, (Wagener et al. 2016).

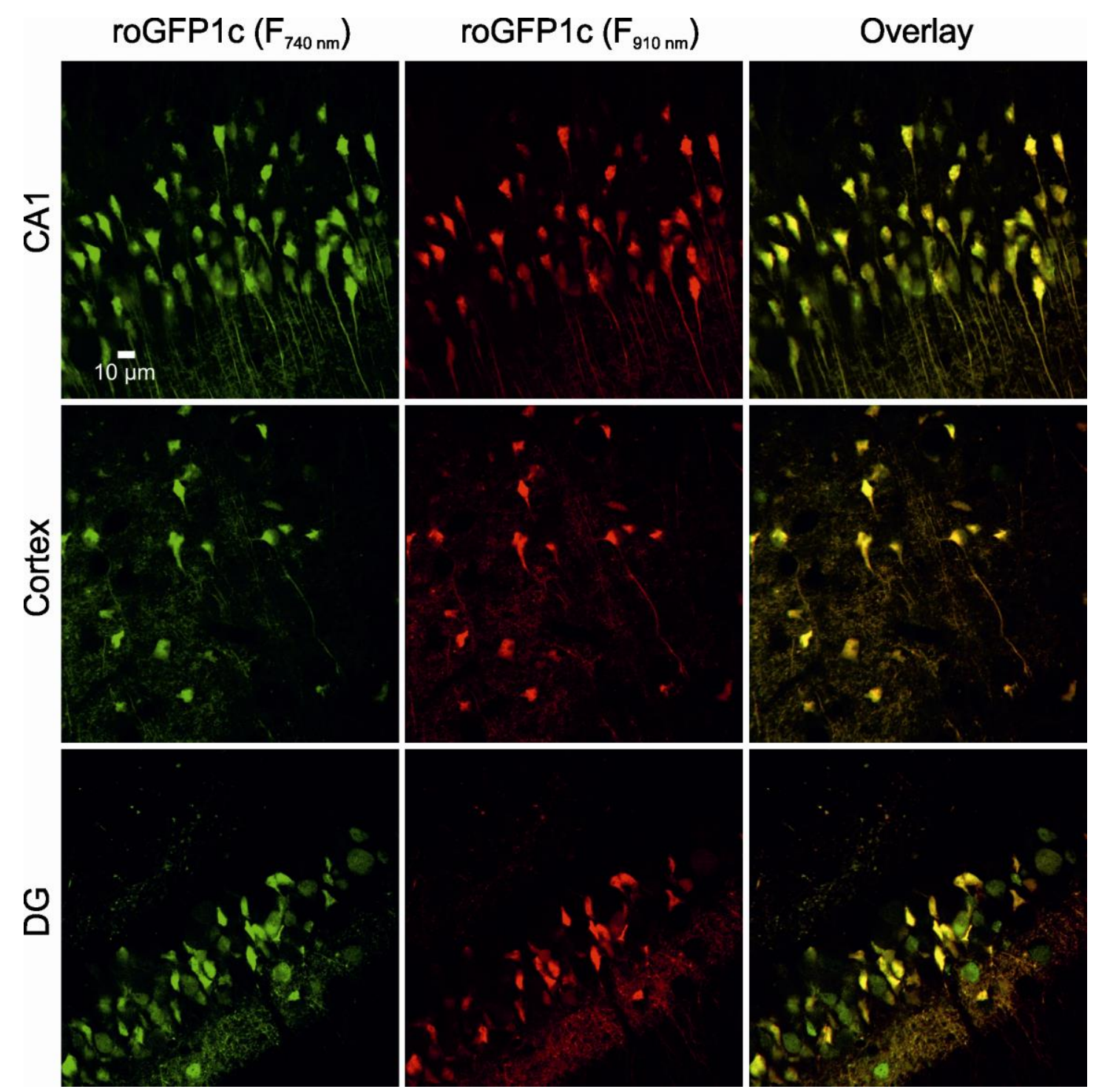

Figure 35. Dual laser ratiometric 2-photon excitation of hippocampal and cortical neurons of roGFP1c transgenic $M_{\text {ecp2 }}{ }^{-y}$ mice.

Cytosolic roGFP1 was homogenously expressed in the cytosol of hippocampal and cortical neurons. For ratiometric redox analyses, the cytosolic roGFP1 was alternatively excited at 
$740 \mathrm{~nm}$ and $910 \mathrm{~nm}$ wavelength by our novel dual laser based excitation ratiometric 2-photon imaging approach. Imaging was performed on acute $400 \mu \mathrm{m}$ thick cortico-hippocampal tissue slices.

This revealed that the cytosolic roGFP1 ratio was more oxidized in roGFP1c Mecp2 ${ }^{-/ y}$ neurons when compared to roGFP1c WT in CA1 $(p<0.01)$ and DG $(p<0.001)$ regions of the hippocampus (Figure 36 , Table 13$)$. In the CA3 subfield and the cortex, those very first tests did not reveal any significant differences of steady state redox balance.

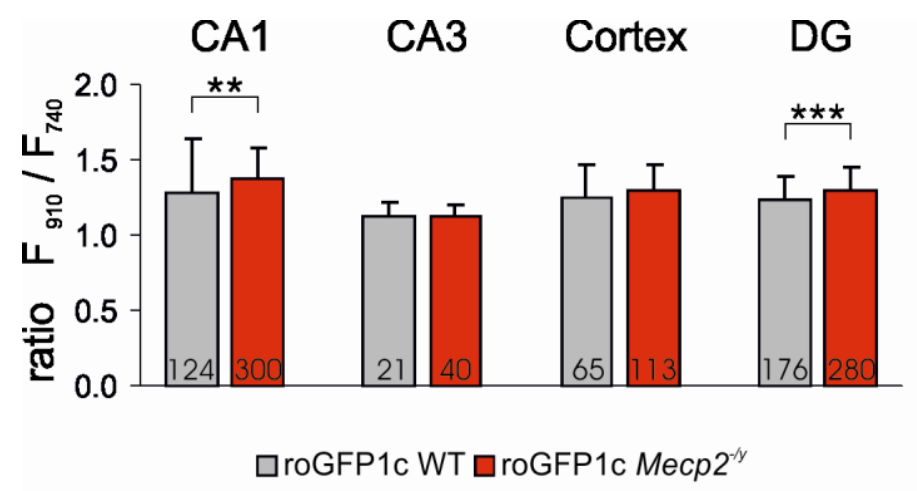

Figure 36. Redox baselines in hippocampal and cortical neurons of adult roGFP1c mice.

The roGFP1c $M_{e c p 2^{-/ y}}$ hippocampal neurons from CA1 and DG regions showed a more oxidized redox steady state balance under control conditions than roGFP1c WT neurons.

Analyses were performed on acute tissue slices. Data were obtained from the very first two roGFPc $M e c p 2^{-/ y}$ mice that became available from the recently started cross-breading of roGFP1c and Rett mice. 


\begin{tabular}{|c|c|c|}
\hline \multicolumn{3}{|c|}{ Evoked changes in roGFP1 ratio $\left(\mathbf{F}_{\mathbf{9 1 0}} / \mathbf{F}_{\mathbf{7 4 0}}\right)$} \\
\cline { 2 - 3 } & roGFP1c WT & ${\text { roGFP1c } \text { Mecp2 }^{-/ y}}^{*}$ \\
\hline \multirow{2}{*}{ CA1 } & $1.3 \pm 0.4 \%$ & $1.4 \pm 0.2 \%$ \\
& $n=124$ & $n=300$ \\
\hline CA3 & $1.1 \pm 0.1 \%$ & $1.1 \pm 0.1 \%$ \\
& $n=21$ & $n=40$ \\
\hline \multirow{2}{*}{ Cortex } & $1.3 \pm 0.2 \%$ & $1.3 \pm 0.2 \%$ \\
& $n=65$ & $n=113$ \\
\hline \multirow{2}{*}{ DG } & $1.2 \pm 0.2 \%$ & $1.3 \pm 0.2 \%$ \\
& $n=176$ & $n=280$ \\
\hline
\end{tabular}

Table 13. RoGFP1 baselines of adult hippocampal and cortical neurons in acute tissue slices of roGFP1c transgenic Mecp2 $^{-/ y}$ mice. 


\section{Discussion}

Changes concerning not only morphology, but also modulating the function of mitochondria were reported in Rett syndrome. These disruptions were obvious in Rett patients and representative mouse models (Ruch et al. 1989; Eeg-Olofsson et al. 1990; Coker and Melnyk 1991; Cornford et al. 1994; Kriaucionis et al. 2006; Gibson et al. 2010; Großer et al. 2012; Pecorelli et al. 2013). However, the previous analyses of mitochondrial metabolism, which had been performed by our group earlier on hippocampal tissue, likely represent mixed neuronal and glial responses from $M e c p 2^{-1 y}$ mice (Großer et al. 2012). Therefore, the aim of the present study was to assess redox changes in mitochondrial and cytosolic compartments specifically in $M e c p 2^{-/ y}$ and WT neurons. Since the hippocampus represents a major structure for studying metabolic and signalling dysfunction as well as neurodegeneration (Wilde et al. 1997; Wang et al. 2007), hippocampal primary cultured neurons as well as hippocampal organotypic slices of $M e c p 2^{-1 y}$ mice were investigated in terms of redox imbalance.

\subsection{The genetically-encoded sensors allow for ratiometric measurements of redox imbalance.}

A wide range of synthetic oxidation-sensitive fluorescent dyes is available to investigate cellular redox changes (Gallop et al. 1984; LeBel et al. 1992; Mohanty et al. 1997). However, these dyes have many disadvantages and limitations, e.g. they respond only to oxidation but not reduction (Foster et al. 2006; Gerich et al. 2009; Funke et al. 2011). More advanced optical sensors such as genetically-encoded optical ROS/redox sensors, are based on fluorescent proteins, in particularl GFP or YFP, and they become redoxsensitive due to an inclusion of reactive cysteine switch, which is inserted closely to the chromophore. While oxidation facilitates forming of a covalent connection between reactive thiols, reduction promotes a breakup of the S-S disulfide bridges. Such fully reversible structural changes modify the fluorescence of the optical redox sensors. Accordingly, these constructs behave as other endogenous redox-sensitive proteins. 
One of the feasible optical redox sensors is HyPer. Based on functional cysteine residues, HyPer allows for the dynamic imaging of sub-cellular $\mathrm{H}_{2} \mathrm{O}_{2}$ levels (Belousov et al. 2006). Previously, HyPer was mostly applied in cell lines, e.g. COS-7, HeLa, PC-12, H4IIE, HThy-ori3.1, HEK 293 but also in rat pancreatic beta-cells primary cultures (Belousov et al. 2006; Markvicheva et al. 2008; Malinouski et al. 2011; Kratschmar et al. 2012; Roma et al. 2012; Weyemi et al. 2012). The detailed response properties of HyPer have been recently investigated in primary cultured hippocampal neurons in mice (Weller et al. 2014).

\subsubsection{Diminished reliability of the redox sensor HyPer}

The first aim of the current thesis was to choose a suitable and reliable redox indicator in order to analyse redox changes within neurons. Using the HyPer sensor would be advantageous to investigate ROS changes within cells as it directly and specifically detects $\mathrm{H}_{2} \mathrm{O}_{2}$ (Belousov et al. 2006). Since chromophores based on YFP are potentially pH-sensitive (Elsliger et al. 1999; Wachter et al. 2000; Belousov et al. 2006; Forkink et al. 2010; Schwarzländer et al. 2011), a crucial goal was to assess the properties of the HyPer sensor. For this, the responses of cytosolic HyPer to various oxidative challenges and different $\mathrm{pH}$ conditions have been evaluated by using the optical $\mathrm{pH}$ sensor SypHer. As shown earlier, this modified redox-insensitive biosensor SypHer can be targeted to the cytosol of hippocampal neurons as well as glial cells, and it reacts only to $\mathrm{pH}$ changes, but no longer to redox modulation (Poburko et al. 2011; Weller et al. 2014).

In the current study, exposure of SypHer-transfected hippocampal primary neurons to different types of stimuli such as propionate, $\mathrm{H}_{2} \mathrm{O}_{2}, \mathrm{tBHP}$, DEDTC, $\mathrm{CN}^{-}$, glutamate, DTT, and hypoxia, caused clear $\mathrm{pH}$ responses. Treatment with a lower concentration $(50 \mu \mathrm{M})$ of $\mathrm{H}_{2} \mathrm{O}_{2}$ induced a slight alkalinization, whereas a higher concentration $(5 \mathrm{mM})$ of $\mathrm{H}_{2} \mathrm{O}_{2}$ resulted in an acidification. Hence, the actual effect of $\mathrm{H}_{2} \mathrm{O}_{2}$ as well as cellular redox changes measured by HyPer upon oxidation might be mimicked by $\mathrm{pH}$ modulations. Interestingly, not only $\mathrm{H}_{2} \mathrm{O}_{2}(50 \mu \mathrm{M})$, but also tBHP, DEDTC and DTT as well as 
reoxygenation after hypoxia revealed similar effects, shifting the SypHer ratio towards alkalinization, whereas $\mathrm{CN}^{-}$, hypoxia and glutamate elicited a marked acidification. Finally, the unequivocal confirmation of $\mathrm{pH}$-sensitivity of the HyPer sensor was obtained with propionate - an intracellular $\mathrm{pH}$ modulator (Sharp and Thomas 1981; Schlue and Thomas 1985) - which caused a marked acidification as confirmed by SypHer responses, and which was followed next by an alkalinization upon washout.

The high pH-sensitivity of HyPer is, however, not the only disadvantage of this redox sensor. Although HyPer has been shown to ensure a pronounced photostability and cell-retention, the unresponsiveness to reducing stimuli questions the reliability of this optical sensor for quantitative redox measurements (Weller et al. 2014).

\subsection{The genetically-encoded optical redox sensor roGFP1 allows for detailed and quantitative monitoring of ROS/redox changes}

Even though recent investigations increased our knowledge on the redox imbalance in Rett syndrome, the detailed role of the cellular redox conditions, and the very contribution of mitochondria to ROS-mediated signalling and pathology are still unclear. Fresh frozen and formalin fixed tissues of brain samples from Rett patients are stored in tissue banks and they are available for research investigations. However, using animal models, which reflect human-related diseases, overcomes many limitations, giving therefore a unique chance to study these disorders on organotypic, cellular and molecular levels. Taking into account, that most of the knowledge about redox modulations in brains of Rett mice has been gathered from mixed responses of neuronal and glial cells, it was important to assess such responses now specifically in Mecp2 $^{-/ y}$ neurons.

Since mitochondria and the cytosol are the main areas, where most metabolic reactions take place within a cell, it was mandatory to investigate redox changes in both of these compartments. Hence, to determine the cellular 
redox imbalance in $\mathrm{Mecp2}^{-/ y}$ mice, the genetically-encoded optical sensor roGFP1 was used to perform quantitative analyses. Similarly to HyPer, roGFP1 is based on thiol groups and functional S-S bridge formation, enabling dynamic and reversible ratiometric measurements of redox modulation (Hanson et al. 2004). In comparison to other redox sensors and synthetic oxidation-sensitive fluorescent dyes, the roGFP1 indicator seems to be one of the most reliable current tools used for redox analyses, since its ratiometric properties cancel out potential artefacts (Bokman and Ward 1981; Funke et al. 2011).

In earlier studies of our group, roGFP1 expression within neurons has been mostly obtained by transfection procedures using lipofectamine (Funke et al. 2011; Großer et al. 2012). In the current thesis, in order to evaluate any defects in mitochondrial function and redox imbalance in $M e c p 2^{-1 y}$ mice, the primary cultured neurons as well as organotypic hippocampal slices have been transduced with viral vectors (AAV-6) to express efficiently the optical sensors mito-roGFP1 and cyto-roGFP1, and to ensure a specific neuronal targeting. Counterlabeling with the neuronal marker MAP2 confirmed the specific localisation of the roGFP1 construct in neuronal cells. Likewise, the mitochondria-specific dye MitoTracker RED FM confirmed the mitochondrial targeting of mito-roGFP1.

Semi-quantitative analyses were possible by taking advantage of the two distinct excitation peaks (395 nm and $470 \mathrm{~nm}$ ) of roGFP1, which respond oppositely to reduction and oxidation (Dooley et al. 2004; Hanson et al. 2004; Funke et al. 2011; Großer et al. 2012). Potential errors, which could arise from diverse fluorophore concentrations and/or illumination intensities, were ruled out by calculating the fluorescence intensity ratio $\left(F_{395} / F_{470}\right)$ (Grynkiewicz et al. 1985). At the very first, roGFP1 had to be calibrated to the specific optical components of the particular experimental set-up and the detailed recording conditions. The states of maximum reduction $(0 \%$ oxidation) and maximum oxidation (100\% oxidation) were forced by applying saturating doses of DTT and $\mathrm{H}_{2} \mathrm{O}_{2}$, respectively (Hanson et al. 2004; Funke et 
al. 2011; Großer et al. 2012). Exposure to reducing agents decreased the roGFP1 fluorescence ratio, which was then increased upon oxidation.

Interestingly, the lowest ratio response of mito-roGFP1 occurred in organotypic hippocampal slices of all preparations. This might be due to a decreased $\mathrm{O}_{2}$ supply bye a diffusional barrier in slice cultures. Moreover, because of a more condensed neuronal network and the presence of glial cells, drug penetration inside the tissue could be slower and/or less efficient than in dissociated cell cultures. Furthermore, the cellular scavenging systems might have already intercepted part of the applied ROS during oxidative challenge before it actually reached the mitochondria. A diminished response range for the mito-roGFP1 ratio in hippocampal slices could have also result from technical aspects such as loss of focus and/or partial shifts of the area of interest due to mitochondrial motility. Indeed, mitochondria are mobile and dynamic organelles with changing shapes and sizes. They might be further modulated by fusion and fission processes. Such mitochondrial fission and arrest of mitochondrial motility have been observed during oxidative stress (Pletjushkina et al. 2006; Gerich et al. 2009; Fan et al. 2010).

\subsection{Redox homeostasis and mitochondrial function are impaired in Mecp2 $^{-/ y}$ neurons}

Modulations not only in the mitochondrial morphology but also in the functional activity of mitochondria have been previously observed in Rett patients' brains (Coker and Melnyk 1991). Reported changes include for example lower levels of cytochrome $c$ oxidase and succinate cytochrome $c$ reductase. Furthermore, recent reports have suggested changes also on the gene level, showing diversities in the expression pattern of genes directly correlated with mitochondrial function (Pecorelli et al. 2013).

Since mitochondria are cellular power houses, the question arose, whether disturbed mitochondrial function might correlate with a lower ATP production and/or lower ATP content. Indeed, Saywell and colleagues showed disruptions in mitochondrial metabolism and reported lower ATP levels in 
brain tissue of $M e c p 2^{-/ y}$ mice (Saywell et al. 2006). However, these results could not be further confirmed by our group for acute adult Mecp2-/y hippocampal slices, which did not contain a lowered cellular ATP content when compared to WT tissue (Fischer et al. 2009). Additionally, more recent findings have demonstrated even increased ATP formation, but also increased ATP consumption in neonatal $\mathrm{Mecp}^{-/ y}$ hippocampus (Toloe et al. 2014). Thus, altered mitochondria might run at higher turn-over rates in Rett mice.

The hypothesis of more intensively working mitochondria in Rett syndrome arose from the findings of Kraucionis and colleagues, who detected significantly increased respiration rates and a larger respiration capacity in $M_{e c p 2^{-/ y}}$ mice (Kriaucionis et al. 2006). Since an appropriate proton gradient needs to be maintained between the intermembrane space and the mitochondrial matrix, the electron transport chain has been suggested to operate at higher rates and to consume more $\mathrm{O}_{2}$ in an attempt to overcome the proton leak across the inner mitochondrial membrane (Kriaucionis et al. 2006). This was also supported by our groups' recent observations of an increased mitochondrial $\mathrm{O}_{2}$ consumption of isolated adult $M e c p 2^{-1 / y}$ hippocampal mitochondria (Menzfeld et al. 2014). Moreover, increased pyruvic and lactic acid levels were found in Rett patients, which might also correlate with functional disturbances in the mitochondrial respiratory chain and the urea cycle (Matsuishi et al. 1992; Dotti et al. 1993; Lappalainen and Riikonen 1994).

Such impaired mitochondria may in consequence activate a vicious circle, leading to additional ROS formation and redox imbalance, and further oxidative stress. In particular, these changes might be even more strengthened due to the impaired breathing in Rett syndrome, e.g. apnoeas, thus causing further cellular network damage and contributing to the disease progression (Figure 37). 


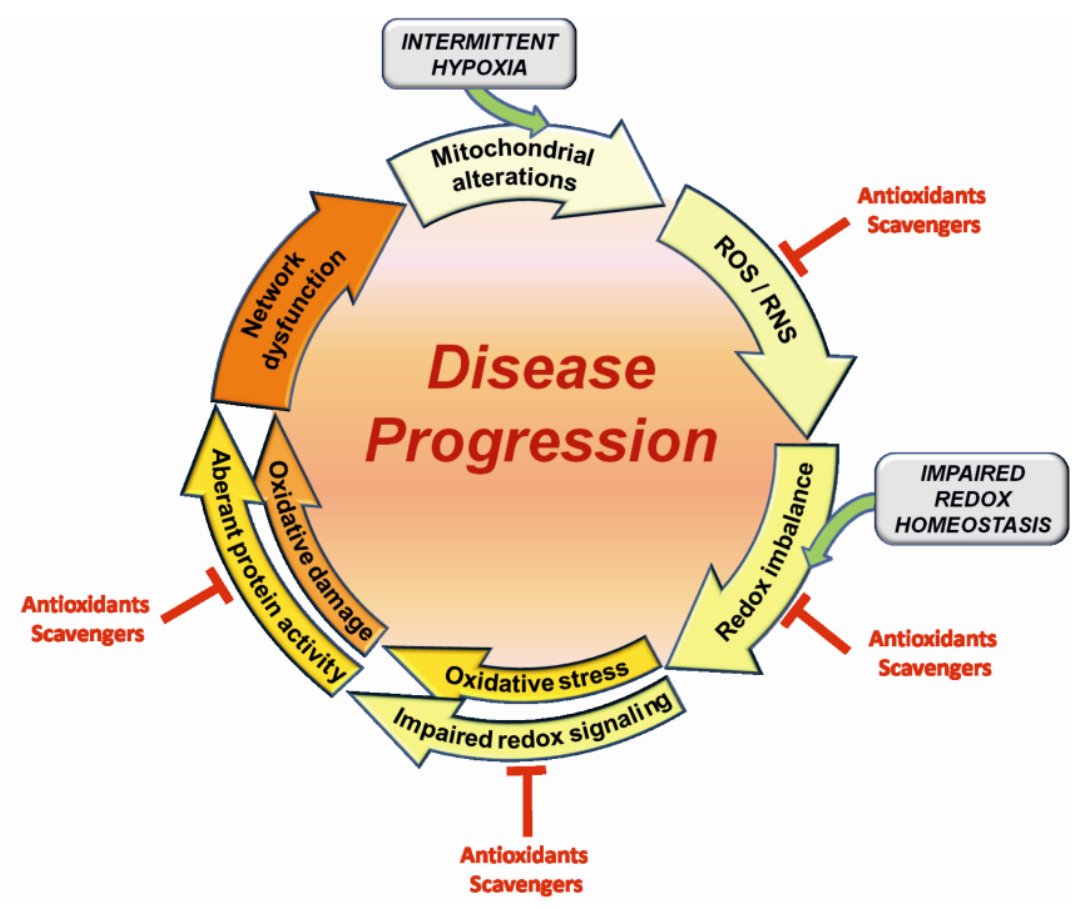

Figure 37. Redox hypothesis of Rett syndrome disease progression.

Functional damage of mitochondria results in more intensified ROS and RNS generation in Rett syndrome. This may contribute to impaired cellular redox signalling and aberrant activity of various cellular proteins such as structural proteins, enzymes, ion channels and neurotransmitter receptors. Redox imbalance and oxidative stress lead further to cellular network dysfunction, thereby accelerating the progression of this neurodevelopmental disease.

This research was originally published in Portland Press Limited. Müller M, Can K. Aberrant redox homoeostasis and mitochondrial dysfunction in Rett syndrome. 2014 Aug; 42(4):95964, Biochemical Society Transactions.

\subsubsection{Mecp2 ${ }^{-/ y}$ neurons are more oxidized already under control conditions}

Changes in redox homeostasis in Rett syndrome have been previously verified by our group (Großer et al. 2012). First, mitochondrial alterations were demonstrated by performing optical imaging and ratiometric analyses of cellular NADH and FAD autofluorescence in acute hippocampal slices of Mecp2 $^{-1 y}$ mice. The elevated FAD/NADH ratio indicates a shift towards oxidation already under control conditions in neonatal (PD 7-10) and adult Mecp2 $2^{-1 y}$ hippocampi (Großer et al. 2012). Furthermore, a more oxidized pretreatment baseline ratio was also detected for $M{ }{ }^{-1 y}$ organotypic hippocampal slices, transfected with cytosolic roGFP1 (Großer et al. 2012). 
Even more oxidizing conditions in $M e c p 2^{-1 y}$ hippocampal tissue have been shown in the absence of B-27 supplement, which contains antioxidants and is commonly used in cell culture media (Großer et al. 2012). However, these results were gained from both glial cells and neurons, and represent responses of a mixed population (Großer et al. 2012).

The present thesis unequivocally confirms that mitochondria of $M e c p 2^{-1 / y}$ neurons suffer from an increased oxidative burden. Indeed, significant changes were found in organotypic hippocampal Mecp2 $2^{-1 y}$ slices, in which the mitochondrial roGFP1 baseline ratio was already markedly increased in comparison to WT hippocampus. A clear increase of redox baseline ratios was also verified for the cytosolic compartment of $M e c p 2^{-1 y}$ slices. Since the cytosol constitutes of a complex mixture of macromolecules, metabolites, ions and other solutes, any changes in the redox conditions might further influence several signalling pathways, which control cellular processes such as cell cycle, cell proliferation, apoptosis, metalloproteinase function, oxygen sensing, protein kinases, phosphatases and transcription factors (Brookes et al. 2004).

\subsubsection{Redox imbalance is more pronounced in somatic areas of hippocampal neurons}

Several studies have previously shown changes in neuronal dendrites during aging and in neurodegeneration. For example, physiological and structural impairments of dendrites include a reduction in arborisation, a lower number of spines or higher mitochondrial oxidative stress (Dickstein et al. 2007; Greenwood et al. 2007; Gomez-Isla et al. 2008; Rocher et al. 2008; Dryanovski et al. 2013; Siskova et al. 2014; Hasel et al. 2015). Interestingly, the current investigation of redox status in $M e c p 2^{-1 / y}$ and WT hippocampal neurons revealed a significantly higher cytosolic baseline roGFP1 ratio in neuronal somata in comparison to dendrites, suggesting a more oxidized basal redox state in the somatic areas. However, neuronal exposure to oxidative challenge by a local neuronal stimulation $\left(\mathrm{H}_{2} \mathrm{O}_{2}, 200 \mu \mathrm{M}, 200 \mathrm{~ms}\right)$, shifted the roGFP1 ratio towards more oxidizing conditions in dendrites of 
these cells, in particular. Similarly to these observations, recent findings have indicated more dramatic fluctuations in the redox potential in cortical dendrites in response to $\mathrm{H}_{2} \mathrm{O}_{2}$ treatment (Hasel et al. 2015). This was confirmed for mouse embryos E17.5 by using the genetically-encoded reporter of the glutathione redox potential Grx1-roGFP2 (Hasel et al. 2015).

Noteworthy, upon $\mathrm{H}_{2} \mathrm{O}_{2}$ administration, Mecp2 $2^{-y}$ neurons showed a tendency towards a higher oxidation in both dendritic and somatic areas as compared to WT. This indicates an increased vulnerability of the cytosolic redox homeostasis in somata and dendrites of $M_{\text {ecp2 }}{ }^{-1 y}$ hippocampus. Indeed, similarly to other ROS, $\mathrm{H}_{2} \mathrm{O}_{2}$ impairs various cellular processes including mitochondrial ATP generation, regulation of $\mathrm{Ca}^{2+}$ levels, activity and gating of ion channels, and the axonal transport of mitochondria (Denu and Tanner 1998; Forte et al. 2007; Marino et al. 2007; Gerich et al. 2009; Fang et al. 2012).

Surprisingly, although $\mathrm{H}_{2} \mathrm{O}_{2}$ was locally applied to dendrites of primary neurons, the first and clear responses to redox modulation were observed rather in the soma. This was true for Mecp2 $2^{-1 y}$ and WT neurons. It is well known that $\mathrm{H}_{2} \mathrm{O}_{2}$ reacts very fast, affecting almost every single molecule. However, although $\mathrm{H}_{2} \mathrm{O}_{2}$ is highly membrane permeable, how exactly it crosses the cellular membrane of neurons, still remains unclear. It was considered that $\mathrm{H}_{2} \mathrm{O}_{2}$ is capable of crossing the membrane of mammalian cells via selected aquaporins (Bienert et al. 2006). Aquaporin-8 (AQP8), which is present in many tissues and organs, was found to have an increased sensitivity to $\mathrm{H}_{2} \mathrm{O}_{2}$ (Bienert et al. 2006). It was shown to be in the plasma membrane, but later studies have indicated that AQP8 is also expressed in the inner mitochondrial membrane (Calamita et al. 2005; Liu et al. 2006; Bienert et al. 2007). Therefore, AQP8 was suggested to be involved in cellular $\mathrm{H}_{2} \mathrm{O}_{2}$ diffusion. On the other hand, recently it has been reported that most neurons in the central nervous system, especially pyramidal neurons, do not express functional aquaporins, which might serve to prevent any unwanted cellular volume changes upon acute osmotic stress (Andrew et al. 2007). 
No matter how $\mathrm{H}_{2} \mathrm{O}_{2}$ crosses the lipid bilayer of neurons, the pathways might differ between dendrites and soma. It would be also interesting to take a closer look at sub-cellular redox buffering capacities within neuronal cells, which might vary in dendritic and somatic compartments, and which may also be different between WT and Mecp2 ${ }^{-/ y}$ mice. On top of that, it would be also tempting to assess, whether the dendritic scavenging systems differ from the somatic ones, with special regards to the activity and localisation of catalase, as it is involved in the degradation of $\mathrm{H}_{2} \mathrm{O}_{2}$ to $\mathrm{H}_{2} \mathrm{O}$ and $\mathrm{O}_{2}$ (Luck 1954).

\subsubsection{Mecp2 $^{-/ y}$ neurons reveal altered responses to redox challenge and show less efficient superoxide dismutation}

It has been previously shown by our group that the roGFP1 ratio was increased under oxidative challenge in $M e c p 2^{-/ y}$ hippocampus (Großer et al. 2012). This finding could be extended and specified in the current work. First, it could be demonstrated that differences in response to redox challenge between Mecp2 $^{-/ y}$ vs. WT mice were obvious especially in hippocampal organotypic slices as compared to neuronal cell culture preparations. It could be also shown that $M e c p 2^{-1 y}$ neurons responded with increased oxidative shifts to $\mathrm{H}_{2} \mathrm{O}_{2}(200 \mu \mathrm{M})$ than WT. This was markedly visible in cytosolic and mitochondrial compartments. However, more excessive oxidation was found rather in cytosol than in mitochondria, suggesting either a higher ROS production and/or a less efficient ROS scavenging, and thus a more pronounced redox imbalance in this compartment. As an intensified extramitochondrial ROS generation in $M e c p 2^{-/ y}$ hippocampus was earlier excluded by our group, and even less pronounced extramitochondrial ROS production was found in Mecp2 $2^{-y}$ neonatal slices (Großer et al. 2012). It therefore seems that especially the redox-maintaining cytosolic buffering systems are impaired in Rett syndrome.

In the current work, differing redox responses of $M e c p 2^{-1 y}$ vs. WT neurons were detected upon SOD inhibition. Previously, the first suggestions towards an impaired SOD in Rett syndrome have been made as a reduced activity of this enzyme was found in blood samples of Rett patients (Sierra et al. 2001). 
Changes in Cu-Zn-SOD (SOD1) - localized in cytoplasm, nucleus and lysosomes of mammalian cells (Chang et al. 1988; Keller et al. 1991; Crapo et al. 1992; Liou et al. 1993; Zelko et al. 2002) - have been also shown on the genetic level, e.g. the SOD1 was upregulated in the adult Mecp ${ }^{-/ y}$ Cornu ammonis 1 (CA1) region of hippocampus (Großer et al. 2012).

Impairment of SOD could be also confirmed specifically in $M e c p 2^{-/ y}$ hippocampal neurons in the current thesis, and this was especially obvious for slice cultures. Upon SOD inhibition, the roGFP1 ratio shifted towards oxidation, which was significantly less intense in $M e c p 2^{-1 /}$ neurons in comparison to WT. This might be due to the assumed reduced activity of SOD in Rett syndrome. Interestingly, these modulations were obvious in cytosol as well as in mitochondria of $M e c p 2^{-1 y}$ hippocampal neurons, thus suggesting alterations not only in cytosolic SOD1 but also in the mitochondrial Mn-SOD (SOD2) (Weisiger and Fridovich 1973; Zelko et al. 2002). Since a mitochondrion is surrounded by two-phospholipid bilayers, weaker responses to SOD inhibition might be an effect of a resistant barrier against drug diffusion. This might also apply to slice culture preparations, since mitochondria of WT and Mecp2 ${ }^{-1 /}$ slices reacted less to SOD inhibition. Mitochondria may be therefore more resistant to SOD blockade.

Also, sub-cellular cyto-roGFP1 ratio changes have been observed for hippocampal neurons in response to SOD inhibition. Interestingly, the DEDTC induced increase of the cyto-roGFP1 ratio was less pronounced in dendrites than in somata, suggesting less efficiency of SOD in dendrites than in the soma of hippocampal neurons. This change was especially clear for WT neurons.

\subsubsection{Mecp2 $^{-/ y}$ hippocampus is more susceptible to hypoxia and mitochondrial challenge}

The $M e c p 2^{-1 y}$ hippocampus has been previously confirmed to be more vulnerable to hypoxia (Fischer et al. 2009; Großer et al. 2012). An increased hypoxia-susceptibility could be now confirmed specifically for $M e c p 2^{-/ y}$ 
neurons. It could be shown that during hypoxia, the roGFP1 ratio decreased more in Mecp2 $^{-/ y}$ hippocampal organotypic slices and neuronal cultures as compared to WT. A substrate reduction and/or inhibition of NOX and/or XO might serve as a possible explanation of this observation. Indeed, NOX and XO have been reported to constitute extramitochondrial ROS sources and were shown to be $\mathrm{O}_{2}$-dependent (Abramov et al. 2007; Funke et al. 2011). Along this line, NOX was suggested to be involved in ROS generation especially during reoxygenation after hypoxia. It was shown that $\mathrm{O}_{2}$ and glucose deprivation as well as reoxygenation led to a cellular $\mathrm{Ca}^{2+}$ increase and further NOX activation (Abramov et al. 2007). Activation of XO also seems to require $\mathrm{Ca}^{2+}$ influx (Dykens et al. 1987). However, other studies questioned the $\mathrm{Ca}^{2+}$-dependent activity of XO (Abramov et al. 2007).

Yet, no clear changes in the roGFP1 ratio traces were observed upon reoxygenation in the current work. Nevertheless, it should be noted that changes in the expression of NOX, XO, ATPase and the rate of mitochondrial depolarization are not stationary processes and they represent an intense and progressive cross-talk between $\mathrm{Ca}^{2+}$, ATP consumption and mitochondrial membrane potential. Therefore, detection of generated ROS depends on how the mitochondrial potential is maintained. Well maintained mitochondria are constantly producing ROS, which might be untraceable though in case of fast depolarizing mitochondria under hypoxic conditions (Abramov et al. 2007). A significant role of mitochondria in hippocampal neurons during hypoxia was also confirmed by the present observations. More pronounced responses to hypoxia were found in mitochondria and cytosol in hippocampal slices, but also in mitochondrial compartments in cell culture, which was more intense in $M e c p 2^{-/ y}$ neurons as compared to WT. Such effects might be also supported by the fact, that the hippocampal network together with the neocortical and cerebellar circuits, constitute the most sensitive parts of the brain to hypoxic/ischemic conditions (Pulsinelli et al. 1982; Schmidt-Kastner and Freund 1991; Fischer et al. 2009).

Furthermore, morphological changes might also enhance hypoxic responses. Smaller and more densely packed neurons as well as less complex dendritic 
arborizations and reduced spine numbers, observed in Rett syndrome (Belichenko et al. 1994; Bauman et al. 1995), could also go in line with a higher hypoxia vulnerability. However, the elevated levels of the hypoxiainducible factor 1-alpha (HIF1 $\alpha$ ) found in hippocampus, neocortex, cerebellum and lower brain stem of $M e c p 2^{-/ y}$ mice, indicated that not only hippocampus, but also other parts of $M e c p 2^{-/ y}$ brain seem to be more hypoxia-sensitive (Fischer et al. 2009).

\subsection{Intact neurotransmission is required for a proper neuronal network functioning}

In spite of the incomplete knowledge on detailed molecular mechanisms, regulating neuronal plasticity, there are indications, pointing to the involvement of neurotransmitters and neurotrophic pathways in neuroplasticity processes (Mattson 2007). Extensive studies have shown a pivotal role of mitochondria in plasticity-related mechanisms such as neuronal polarity formation and neurite outgrowth control (Vayssiere et al. 1992; Mattson and Partin 1999; Chada and Hollenbeck 2004; Lee and Peng 2006). Also, damaged mitochondria contribute to changes in neuronal plasticity as ROS serve signalling functions by modulating activities of kinases or the phosphatase calcineurin (Kamsler and Segal 2003; Hongpaisan et al. 2004).

Since the extracellular electrical stimulation of primary neurons was not suitable to perform detailed studies on neuronal activity, primary neurons were exposed to various neurotransmitters. It is well known that exaggerated ROS production, redox imbalance and further aberrant energy states, lead to neuronal network disturbances and functional changes within cells. Data on neuronal responses to glutamate, norepinephrine, serotonin and dopamine confirmed such disruptions by shifting the cellular redox conditions towards oxidation. With all transmitters tested, this was significantly more pronounced in $M e c p 2^{-/ y}$ than in WT neurons.

The current thesis is the first study showing very clear redox responses of Mecp2 ${ }^{-1 y}$ neurons to neurotransmitters, which additionally confirms the cellular 
redox impairment in $M e c p 2^{-1 / y}$ hippocampus. Therefore, another aim of the current work was to evaluate possible candidate processes, which might stand behind such redox alterations, e.g. activation of $\mathrm{NOX}$ and $\mathrm{XO}, \mathrm{Ca}^{2+}$ influx, engagement of mitochondrial respiratory chain or increase of cAMP levels. Since glutamate is the principal excitatory neurotransmitter and mediates also neuronal excitotoxicity when excessively released (Atlante et al. 2001), it was interesting to assess the possible signalling pathways of redox changes in response to glutamate. Also, the responses to dopamine under above-mentioned conditions have been investigated as dopamine was the only one, which evoked redox responses with full recovery in $M e c p 2^{-y}$ and WT neurons.

\subsubsection{Activation of NADPH oxidase and xanthine oxidase contributes to ROS production in response to glutamate}

Disruptions in various neurotransmitters have been already proposed for Rett syndrome (Hamberger et al. 1992; Lappalainen and Riikonen 1996; Viemari et al. 2005; Chao et al. 2010; Maezawa and Jin 2010). In the current thesis, it became evident that exposure to neurotransmitters glutamate, norepinephrine, serotonin and dopamine, induced redox alterations and shifted redox status towards more oxidizing conditions. It was especially $M_{e c p 2^{-/ y}}$ neurons, which overreacted and which did not show a proper recovery of the cyto-roGFP1 ratio to its pre-treatment baseline upon wash out of the tested compounds. This might suggest a persistent redox impairment in $M_{e c p 2^{-1 y}}$ neurons. Such changes have been particularly obvious during glutamate application as the cyto-roGFP1 ratio continued to increase even when the treatment was terminated.

Glutamate has been previously shown to induce an irreversible neuronal injury, leading to cell death (Choi 1988; Hamberger et al. 1992; Lappalainen and Riikonen 1996; Simonian and Coyle 1996; Meldrum 2000; Ha et al. 2009; Maezawa and Jin 2010). It was suggested to activate lactate production, causing neuronal acidification and damaging mitochondria by decreasing mitochondrial membrane potential and provoking mitochondrial uncoupling, 
which gives rise to ROS generation. Moreover, a decrease in the efficiency of the cellular antioxidant defences by glutamate has been shown to enhance an oxidative stress (Ciani et al. 1996). Surprisingly, treatment with glutamate, in the presence of the mitochondrial uncoupler FCCP, failed to prevent the increase in cyto-roGFP1 ratio. This strongly suggests that the major redox response to glutamate is mediated by other cellular sources rather than mitochondria. However, it should be mentioned that mitochondrial uncoupling evoked the largest decrease of the cyto-roGFP1 ratio baseline in WT and $M{ }^{2} 2^{-1 y}$ neurons when compared to ACSF conditions. Therefore, it indicates that mitochondria do contribute to redox changes and ROS production in hippocampal neurons, at least under control baseline conditions.

Glutamate was proposed to mediate its effects on different molecular levels, namely through ionotropic receptors, i.e. NMDA, $\alpha$-amino-3-hydroxy-5-methyl4-isoxazolepropionic acid (AMPA) and kainate receptors in postsynapses (Nicholls and Budd 2000). Activation of NMDA and non-NMDA receptors leads to a neuronal injury due to intracellular $\mathrm{Ca}^{2+}$ overload (Olney 1969; Michaels and Rothman 1990; Nicholls and Budd 2000). In the current study, the cultured $M e c p 2^{-/ y}$ neurons still markedly reacted to glutamate in $\mathrm{Ca}^{2+}$ free conditions, shifting the cyto-roGFP1 ratio towards oxidation. However, the responses of WT neurons to glutamate were almost abolished.

Glutamate may also operate through G-protein coupled glutamate metabotropic receptors (mGluR) by affecting ion channels or secondary messengers such as diacylglycerol and cAMP (Schoepp and Conn 1993; Pin and Duvoisin 1995; Conn and Pin 1997; Meldrum 2000). Yet, activation of the adenylate cyclase and hence, elevation of the intracellular CAMP concentration by forskolin, did not reveal any changes in the cyto-roGFP1 ratio, which excludes an involvement of cAMP-coupled mGluR in redox modulation during neuronal exposure to glutamate.

Interestingly, it could be also shown, that upon blocking NOX by DPI, glutamate did not cause any longer major redox changes. This indicates that NOX might be crucially engaged in redox responses to glutamate. The NOX 
was earlier proposed to intensify ROS production by neurons, whereas the generation of $\mathrm{O}_{2}^{-}$was restrained upon NOX inhibition in the presence of glutamate (Brennan et al. 2009). Furthermore, ROS formation induced by NOX under glutamate administration, seemed to be independent on mitochondria. Neurons have been confirmed to generate ROS even upon an overexpression of the mitochondrial SOD2, whereas ROS output was markedly reduced in neurons lacking NOX (Brennan et al. 2009; Demaurex and Scorrano 2009).

Nevertheless, other studies suggested also a role of $\mathrm{XO}$ in the production of ROS due to glutamate neurotoxicity in cerebellar granule cells. This occurs via a conversion of xanthine dehydrogenase $(\mathrm{XDH})$ to $\mathrm{XO}$ in response to $\mathrm{Ca}^{2+}$ influx (Atlante et al. 1997). Moreover, generation of peroxide was previously shown to be prevented by laupeptin, which inhibits the conversion of XDH to XO (Atlante et al. 1997). Indeed, the inhibition of XO by allopurinol prevented glutamate-mediated changes in the roGFP1 ratio, confirming a crucial XO involvement in these redox responses.

It should be mentioned, that $\mathrm{XO}$ seems to act as the most efficient catalyzer of. $\mathrm{O}_{2}^{-}$production, since the time course of $\mathrm{XO}$ activity coincides with the generation of oxygen radicals after glutamate stimulation of cerebellar granule cells (Atlante et al. 1997). On top of that, the same studies showed that XO activity was lost in the presence of the NMDA receptor antagonist MK-801 or ethylene glycol tetraacetic acid (EGTA), which captures $\mathrm{Ca}^{2+}$. This suggests that activation of $\mathrm{XO}$, and therefore, conversion of $\mathrm{XDH}$ to $\mathrm{XO}$, is mediated by NMDA receptors and $\mathrm{Ca}^{2+}$ influx. Thus, $\mathrm{Ca}^{2+}$ influx is a crucial trigger of $\mathrm{ROS}$ production as it may activate $\mathrm{XO}$ and NOX. This view is supported by the observation that WT neurons do not longer become oxidized when glutamate is applied under $\mathrm{Ca}^{2+}$ free conditions. However, the latest studies showed that NOX may be also active even in low magnesium $\left(\mathrm{Mg}^{2+}\right)$ and $\mathrm{Ca}^{2+}$-free conditions (Kovac et al. 2014).

It should be mentioned, that glutamate was the only neurotransmitter, which induced an initial drop in the cyto-roGFP1 ratio traces. The reason of this 
response changes is, however, not clear. It could be that there are other glutamate receptor subtypes, which mediate this only moderate response.

\subsubsection{Dopamine modulates mitochondrial function in $\mathrm{Mecp2}^{-/ y}$ hippocampal neurons}

The link between neuron-specific oxidative stress and dopamine was previously indicated by several studies (Cubells et al. 1994; Wrona et al. 1997; Cadet and Brannock 1998; Miyazaki and Asanuma 2008). In the current thesis, it was shown that clear cytosolic redox changes could be evoked by the dopaminergic system in $M e c p 2^{-/ y}$ and WT hippocampal neurons. Indeed, dopaminergic neurotoxicity was proposed to be strictly related with the generation of ROS and dopamine quinine formation (Graham 1978; Sulzer et al. 2000). Since dopaminergic neurons are enriched with iron and other transition metals, $\mathrm{H}_{2} \mathrm{O}_{2}$ may lead to the production of $\cdot \mathrm{OH}$ in a Fenton reaction. Moreover, dopamine was suggested to provoke the mitochondrial impairment, e.g. dopamine has been shown to inhibit the mitochondrial complex I, demolish mitochondrial motility and dissipate the mitochondrial membrane potential (Ben-Shachar 2002; Brenner-Lavie et al. 2008; Miyazaki and Asanuma 2008). Such changes clearly suggest that dopamine indeed interacts with the mitochondrial respiration and may lead to cellular redox impairment. However, since dopamine shifted the roGFP1 ratio towards oxidation despite the presence of FCCP, mitochondria are not the only molecular player through which dopamine acts, although their contribution seemed to be higher in $\mathrm{Mecp}^{-/ y}$ than in WT neurons. The $\mathrm{Ca}^{2+}$ free conditions did not prevent an increased cyto-roGFP1 ratio upon dopamine application, suggesting $\mathrm{Ca}^{2+}$ release from other cellular sources. Interestingly, the roGFP1 responses to dopamine were markedly dampened when NOX was inhibited by DPI in the current thesis, which again indicated a pronounced role of NOX in ROS producing signalling pathways.

Yet, the question remains, whether such pronounced responses of the cytoroGFP1 ratio were simply due to high concentration of dopamine applied, as a concentration of $30 \mathrm{nM}$ is considered to be within the physiological range 
(Lee et al. 2000). Nevertheless, the $500 \mu \mathrm{M}$ dopamine used here, can be considered not to impair cell viability as the redox changes fully recovered upon wash-out. Also, studies on schizophrenia revealed that intact, coupled and respiring mitochondria could accumulate saturated doses of dopamine, and non-lethal dopamine-mitochondria interactions were detected (BrennerLavie et al. 2008).

\section{Redox imbalance occurs not only in $M e c p 2^{-/ y}$ neonates but also in neurons of adult and symptomatic Rett mice}

Transgenic mice, carrying specifically mitochondrial (roGFP1m) and cytosolic (roGFP1c) roGFP1 sensors within neurons, could be recently successfully generated in our lab (Wagener et al. 2016). Cross-breeding of these mice with heterozygote $M e c p 2^{+/ y}$ females will allow to obtain symptomatic Mecp2-ly mice, which carry roGFP1 redox indicators for mitochondria and cytosol, specifically in neurons.

In the current work, the first tests towards an investigation of a redox imbalance under control conditions in hippocampal and cortical neurons of adult roGFP1c Mecp2 ${ }^{-/ y}$ mice could be performed. It could be confirmed, that roGFP1c Mecp2 $2^{-/ y}$ neurons (PD 47-52) of CA1 and DG regions of hippocampus were more oxidized as compared to the respective WT mice. Although a proper evaluation of neuronal responses to oxidative challenge conditions is lacking so far, these are the first tests confirming redox impairment by using functional redox imaging in living neurons of symptomatic, adult Rett mice. 


\section{Conclusion and future perspectives}

Extensive research has been carried out to evaluate the redox changes in $\mathrm{Mecp2}^{-/ y}$ neonatal hippocampus by taking advantage of the geneticallyencoded optical sensor roGFP1. In the current thesis, it could be shown that $M e c p 2^{-/ y}$ hippocampal organotypic slices suffer from an increased oxidative burden, which has been confirmed also for $M \mathrm{Cp}^{-/ y}$ dissociated neurons. They were more susceptible to hypoxia and revealed more intense oxidizing shifts in response to oxidative challenge. Moreover, Mecp2 $^{-/ y}$ neurons possess less efficiently working cellular scavenging systems.

Upon redox challenge, genotypically different redox responses could be found in the cytosol as well as at the level of mitochondria. This confirms that Rett syndrome is indeed associated with early mitochondrial alterations. Although there are studies questioning that Rett syndrome constitutes a primary mitochondrial disease (Matsuishi et al. 2011), it could be confirmed that neuronal mitochondria of $M e c p 2^{-1 / y}$ hippocampus do show a more oxidized and a more vulnerable redox balance already at neonatal and presymptomatic stages. These findings therefore support the hypothesis, that mitochondria constitute a primary cause or at least very crucial events for disease progression.

Furthermore, the current thesis underlines the key role of neurotransmitters in neuronal redox events and provides evidence that even subtle changes in the neuronal activity may lead to a substantially modified redox balance. However, more knowledge must be gathered in order to better understand the fine molecular mechanisms by which neurotransmitters lead to intensified ROS production and cellular redox changes. Without doubt, the exaggerated redox responses seen in $M e c p 2^{-1 y}$ neurons with all neurotransmitters tested, may shed a new light on disturbed neurotransmission in Rett syndrome. So far, the responses to the tested neurotransmitters have been assessed in individual $\mathrm{Mecp2}^{-1 /}$ cultured neurons. Now, it would be interesting to investigate similar reactions in organotypic or even acute hippocampal $M e c p 2^{-1 y}$ slices. It would be evenly tempting to assess redox changes in the 
mitochondrial compartments of $M e c p 2^{-/ y}$ and WT neurons under the treatment with these neurotransmitters.

Transfection and viral transduction procedures required to deliver roGFP1coding DNA limited the current studies to cell and slice cultures, and hence neonates. The next crucial step would be therefore to use the novel transgenic redox-sensor mice. They specifically express roGFP1 in the cytosol and the mitochondrial matrix, and they will finally enable sub-cellular analyses of redox changes also in adult neurons (Wagener et al. 2016). Cross-breeding these transgenic lines with the $M e c p 2^{-1 y}$ mice will provide the tremendous opportunity to assess redox imbalance of adult stages of $M_{e c p 2^{-/ y}}$ mice and to correlate it in detail with the disease progression of Rett syndrome. 


\section{References}

Abramov A. Y., Scorziello A. and Duchen M. R. (2007) Three distinct mechanisms generate oxygen free radicals in neurons and contribute to cell death during anoxia and reoxygenation. $J$ Neurosci 27, 1129-1138.

Acampa M. and Guideri F. (2006) Cardiac disease and Rett syndrome. Arch Dis Child 91, 440-443.

Amir R. E., Van den Veyver I. B., Wan M., Tran C. Q., Francke U. and Zoghbi H. Y. (1999) Rett syndrome is caused by mutations in X-linked MECP2, encoding methyl-CpG-binding protein 2. Nat Genet 23, 185-188.

Andrew R. D., Labron M. W., Boehnke S. E., Carnduff L. and Kirov S. A. (2007) Physiological evidence that pyramidal neurons lack functional water channels. Cereb Cortex 17, 787-802.

Archer H. L., Evans J., Edwards S., Colley J., Newbury-Ecob R., O'Callaghan F., Huyton M., O'Regan M., Tolmie J., Sampson J., Clarke A. and Osborne J. (2006) CDKL5 mutations cause infantile spasms, early onset seizures, and severe mental retardation in female patients. J Med Genet 43, 729-734.

Archer H. L., Whatley S. D., Evans J. C., Ravine D., Huppke P., Kerr A., Bunyan D., Kerr B., Sweeney E., Davies S. J., Reardon W., Horn J., MacDermot K. D., Smith R. A., Magee A., Donaldson A., Crow Y., Hermon G., Miedzybrodzka Z., Cooper D. N., Lazarou L., Butler R., Sampson J., Pilz D. T., Laccone F. and Clarke A. J. (2006) Gross rearrangements of the MECP2 gene are found in both classical and atypical Rett syndrome patients. J Med Genet 43, 451-456.

Ariani F., Hayek G., Rondinella D., Artuso R., Mencarelli M. A., SpanholRosseto A., Pollazzon M., Buoni S., Spiga O., Ricciardi S., Meloni I., Longo I., Mari F., Broccoli V., Zappella M. and Renieri A. (2008) FOXG1 is responsible for the congenital variant of Rett syndrome. Am J Hum Genet 83, 89-93.

Armstrong D. D. (2005) Neuropathology of Rett syndrome. J Child Neurol 20, 747-753.

Atlante A., Gagliardi S., Minervini G. M., Ciotti M. T., Marra E. and Calissano P. (1997) Glutamate neurotoxicity in rat cerebellar granule cells: a major role for xanthine oxidase in oxygen radical formation. J Neurochem 68, 20382045.

Atlante A., Calissano P., Bobba A., Giannattasio S., Marra E. and Passarella S. (2001) Glutamate neurotoxicity, oxidative stress and mitochondria. FEBS Lett 497, 1-5.

Balmer D., Goldstine J., Rao Y. M. and LaSalle J. M. (2003) Elevated methylCpG-binding protein 2 expression is acquired during postnatal human brain development and is correlated with alternative polyadenylation. $J$ Mol Med 81, 61-68. 
Bauman M. L., Kemper T. L. and Arin D. M. (1995) Pervasive neuroanatomic abnormalities of the brain in three cases of Rett's syndrome. Neurology 45, 1581-1586.

Belichenko P. V. and Dahlstrom A. (1995) Studies on the 3-dimensional architecture of dendritic spines and varicosities in human cortex by confocal laser scanning microscopy and Lucifer yellow microinjections. J Neurosci Methods 57, 55-61.

Belichenko P. V., Oldfors A., Hagberg B. and Dahlstrom A. (1994) Rett syndrome: 3-D confocal microscopy of cortical pyramidal dendrites and afferents. Neuroreport 5, 1509-1513.

Belichenko P. V., Wright E. E., Belichenko N. P., Masliah E., Li H. H., Mobley W. C. and Francke U. (2009) Widespread changes in dendritic and axonal morphology in Mecp2-mutant mouse models of Rett syndrome: evidence for disruption of neuronal networks. J Comp Neurol 514, 240-258.

Belousov V. V., Fradkov A. F., Lukyanov K. A., Staroverov D. B., Shakhbazov K. S., Terskikh A. V. and Lukyanov S. (2006) Genetically encoded fluorescent indicator for intracellular hydrogen peroxide. Nat Methods 3, 281-286.

Ben-Shachar D. (2002) Mitochondrial dysfunction in schizophrenia: a possible linkage to dopamine. J Neurochem 83, 1241-1251.

Bienert G. P., Schjoerring J. K. and Jahn T. P. (2006) Membrane transport of hydrogen peroxide. Biochim Biophys Acta 1758, 994-1003.

Bienert G. P., Moller A. L., Kristiansen K. A., Schulz A., Moller I. M., Schjoerring J. K. and Jahn T. P. (2007) Specific aquaporins facilitate the diffusion of hydrogen peroxide across membranes. J Biol Chem 282, 11831192.

Bokman S. H. and Ward W. W. (1981) Renaturation of Aequorea greefluorescent protein. Biochem Biophys Res Commun 101, 1372-1380.

Boveris A. and Chance B. (1973) The mitochondrial generation of hydrogen peroxide. General properties and effect of hyperbaric oxygen. Biochem J 134, 707-716.

Boveris A., Oshino N. and Chance B. (1972) The cellular production of hydrogen peroxide. Biochem J 128, 617-630.

Brand M. D. (2010) The sites and topology of mitochondrial superoxide production. Exp Gerontol 45, 466-472.

Brennan A. M., Suh S. W., Won S. J., Narasimhan P., Kauppinen T. M., Lee H., Edling Y., Chan P. H. and Swanson R. A. (2009) NADPH oxidase is the primary source of superoxide induced by NMDA receptor activation. Nat Neurosci 12, 857-863. 
Brenner-Lavie H., Klein E., Zuk R., Gazawi H., Ljubuncic P. and Ben-Shachar D. (2008) Dopamine modulates mitochondrial function in viable SH-SY5Y cells possibly via its interaction with complex I: relevance to dopamine pathology in schizophrenia. Biochim Biophys Acta 1777, 173-185.

Brookes P. S., Yoon Y., Robotham J. L., Anders M. W. and Sheu S. S. (2004) Calcium, ATP, and ROS: a mitochondrial love-hate triangle. Am J Physiol Cell Physiol 287, C817-833.

Brucke T., Sofic E., Killian W., Rett A. and Riederer P. (1987) Reduced concentrations and increased metabolism of biogenic amines in a single case of Rett-syndrome: a postmortem brain study. J Neural Transm 68, 315-324.

Cadet J. L. and Brannock C. (1998) Free radicals and the pathobiology of brain dopamine systems. Neurochem Int 32, 117-131.

Calamita G., Ferri D., Gena P., Liquori G. E., Cavalier A., Thomas D. and Svelto M. (2005) The inner mitochondrial membrane has aquaporin-8 water channels and is highly permeable to water. J Biol Chem 280, 17149-17153.

Calfa G., Percy A. K. and Pozzo-Miller L. (2011) Experimental models of Rett syndrome based on Mecp2 dysfunction. Exp Biol Med (Maywood) 236, 3-19.

Chada S. R. and Hollenbeck P. J. (2004) Nerve growth factor signaling regulates motility and docking of axonal mitochondria. Curr Biol 14, 1272 1276.

Chahrour M. and Zoghbi H. Y. (2007) The story of Rett syndrome: from clinic to neurobiology. Neuron 56, 422-437.

Chahrour M., Jung S. Y., Shaw C., Zhou X., Wong S. T., Qin J. and Zoghbi H. Y. (2008) MeCP2, a key contributor to neurological disease, activates and represses transcription. Science 320, 1224-1229.

Chan P. H. (2001) Reactive oxygen radicals in signaling and damage in the ischemic brain. J Cereb Blood Flow Metab 21, 2-14.

Chang L. Y., Slot J. W., Geuze H. J. and Crapo J. D. (1988) Molecular immunocytochemistry of the CuZn superoxide dismutase in rat hepatocytes. $J$ Cell Biol 107, 2169-2179.

Chao H. T., Chen H., Samaco R. C., Xue M., Chahrour M., Yoo J., Neul J. L., Gong S., Lu H. C., Heintz N., Ekker M., Rubenstein J. L., Noebels J. L., Rosenmund C. and Zoghbi H. Y. (2010) Dysfunction in GABA signalling mediates autism-like stereotypies and Rett syndrome phenotypes. Nature 468, 263-269.

Chen C. S. and Gee K. R. (2000) Redox-dependent trafficking of 2,3,4,5, 6pentafluorodihydrotetramethylrosamine, a novel fluorogenic indicator of cellular oxidative activity. Free Radic Biol Med 28, 1266-1278. 
Chen R. Z., Akbarian S., Tudor M. and Jaenisch R. (2001) Deficiency of methyl-CpG binding protein-2 in CNS neurons results in a Rett-like phenotype in mice. Nat Genet 27, 327-331.

Choi D. W. (1988) Glutamate neurotoxicity and diseases of the nervous system. Neuron 1, 623-634.

Christodoulou J., Grimm A., Maher T. and Bennetts B. (2003) RettBASE: The IRSA MECP2 variation database-a new mutation database in evolution. Hum Mutat 21, 466-472.

Ciani E., Groneng L., Voltattorni M., Rolseth V., Contestabile A. and Paulsen R. E. (1996) Inhibition of free radical production or free radical scavenging protects from the excitotoxic cell death mediated by glutamate in cultures of cerebellar granule neurons. Brain Res 728, 1-6.

Clayton-Smith J., Watson P., Ramsden S. and Black G. C. (2000) Somatic mutation in MECP2 as a non-fatal neurodevelopmental disorder in males. Lancet 356, 830-832.

Cohen D. R., Matarazzo V., Palmer A. M., Tu Y., Jeon O. H., Pevsner J. and Ronnett G. V. (2003) Expression of MeCP2 in olfactory receptor neurons is developmentally regulated and occurs before synaptogenesis. Mol Cell Neurosci 22, 417-429.

Coker S. B. and Melnyk A. R. (1991) Rett syndrome and mitochondrial enzyme deficiencies. J Child Neurol 6, 164-166.

Conn P. J. and Pin J. P. (1997) Pharmacology and functions of metabotropic glutamate receptors. Annu Rev Pharmacol Toxicol 37, 205-237.

Cornford M. E., Philippart M., Jacobs B., Scheibel A. B. and Vinters H. V. (1994) Neuropathology of Rett syndrome: case report with neuronal and mitochondrial abnormalities in the brain. J Child Neurol 9, 424-431.

Crapo J. D., Oury T., Rabouille C., Slot J. W. and Chang L. Y. (1992) Copper,zinc superoxide dismutase is primarily a cytosolic protein in human cells. Proc Natl Acad Sci U S A 89, 10405-10409.

Cubells J. F., Rayport S., Rajendran G. and Sulzer D. (1994) Methamphetamine neurotoxicity involves vacuolation of endocytic organelles and dopamine-dependent intracellular oxidative stress. J Neurosci 14, 2260 2271.

De Felice C., Signorini C., Leoncini S., Pecorelli A., Durand T., Valacchi G., Ciccoli L. and Hayek J. (2012) The role of oxidative stress in Rett syndrome: an overview. Ann N Y Acad Sci 1259, 121-135.

De Felice C., Ciccoli L., Leoncini S., Signorini C., Rossi M., Vannuccini L., Guazzi G., Latini G., Comporti M., Valacchi G. and Hayek J. (2009) Systemic oxidative stress in classic Rett syndrome. Free Radic Biol Med 47, 440-448. 
de la Monte S. M., Luong T., Neely T. R., Robinson D. and Wands J. R. (2000) Mitochondrial DNA damage as a mechanism of cell loss in Alzheimer's disease. Lab Invest 80, 1323-1335.

Dean R. T., Fu S., Stocker R. and Davies M. J. (1997) Biochemistry and pathology of radical-mediated protein oxidation. Biochem $\mathrm{J}$ 324, 1-18.

Deguchi K., Antalffy B. A., Twohill L. J., Chakraborty S., Glaze D. G. and Armstrong D. D. (2000) Substance P immunoreactivity in Rett syndrome. Pediatr Neurol 22, 259-266.

Demaurex N. and Scorrano L. (2009) Reactive oxygen species are NOXious for neurons. Nat Neurosci 12, 819-820.

Demchenko I. T., Oury T. D., Crapo J. D. and Piantadosi C. A. (2002) Regulation of the brain's vascular responses to oxygen. Circ Res 91, 10311037.

Denu J. M. and Tanner K. G. (1998) Specific and reversible inactivation of protein tyrosine phosphatases by hydrogen peroxide: evidence for a sulfenic acid intermediate and implications for redox regulation. Biochemistry 37, 5633-5642.

Dickstein D. L., Kabaso D., Rocher A. B., Luebke J. I., Wearne S. L. and Hof P. R. (2007) Changes in the structural complexity of the aged brain. Aging Cell 6, 275-284.

Dooley C. T., Dore T. M., Hanson G. T., Jackson W. C., Remington S. J. and Tsien R. Y. (2004) Imaging dynamic redox changes in mammalian cells with green fluorescent protein indicators. J Biol Chem 279, 22284-22293.

Dotti M. T., Manneschi L., Malandrini A., De Stefano N., Caznerale F. and Federico A. (1993) Mitochondrial dysfunction in Rett syndrome. An ultrastructural and biochemical study. Brain Dev 15, 103-106.

Dryanovski D. I., Guzman J. N., Xie Z., Galteri D. J., Volpicelli-Daley L. A., Lee V. M., Miller R. J., Schumacker P. T. and Surmeier D. J. (2013) Calcium entry and alpha-synuclein inclusions elevate dendritic mitochondrial oxidant stress in dopaminergic neurons. J Neurosci 33, 10154-10164.

Duchen M. R. (2000) Mitochondria and calcium: from cell signalling to cell death. J Physiol 529, 57-68.

Dugan L. L., Sensi S. L., Canzoniero L. M., Handran S. D., Rothman S. M., Lin T. S., Goldberg M. P. and Choi D. W. (1995) Mitochondrial production of reactive oxygen species in cortical neurons following exposure to $\mathrm{N}$-methyl-Daspartate. J Neurosci 15, 6377-6388.

Dykens J. A., Stern A. and Trenkner E. (1987) Mechanism of kainate toxicity to cerebellar neurons in vitro is analogous to reperfusion tissue injury. $J$ Neurochem 49, $1222-1228$. 
Eeg-Olofsson O., al-Zuhair A. G., Teebi A. S., Daoud A. S., Zaki M., Besisso M. S. and Al-Essa M. M. (1990) Rett syndrome: a mitochondrial disease? J Child Neurol 5, 210-214.

Elsliger M. A., Wachter R. M., Hanson G. T., Kallio K. and Remington S. J. (1999) Structural and spectral response of green fluorescent protein variants to changes in $\mathrm{pH}$. Biochemistry 38, 5296-5301.

Engineer C. T., Rahebi K. C., Borland M. S., Buell E. P., Centanni T. M., Fink M. K., Im K. W., Wilson L. G. and Kilgard M. P. (2015) Degraded neural and behavioral processing of speech sounds in a rat model of Rett syndrome. Neurobiol Dis 83, 26-34.

Esposti M. D., Hatzinisiriou I., McLennan H. and Ralph S. (1999) Bcl-2 and mitochondrial oxygen radicals. New approaches with reactive oxygen species-sensitive probes. J Biol Chem 274, 29831-29837.

Fan X., Hussien R. and Brooks G. A. (2010) $\mathrm{H}_{2} \mathrm{O}_{2}$-induced mitochondrial fragmentation in $\mathrm{C}_{2} \mathrm{C}_{12}$ myocytes. Free Radic Biol Med 49, 1646-1654.

Fang C., Bourdette D. and Banker G. (2012) Oxidative stress inhibits axonal transport: implications for neurodegenerative diseases. Mol Neurodegener 7, 29.

Fernández-Checa J. C., Fernández A., Morales A., Mari M., Garcia-Ruiz C. and Colell A. (2010) Oxidative stress and altered mitochondrial function in neurodegenerative diseases: lessons from mouse models. CNS Neurol Disord Drug Targets 9, 439-454.

Finkel T. (2011) Signal transduction by reactive oxygen species. J Cell Biol 194, 7-15.

Fischer M., Reuter J., Gerich F. J., Hildebrandt B., Hägele S., Katschinski D. and Müller M. (2009) Enhanced hypoxia susceptibility in hippocampal slices from a mouse model of Rett syndrome. J Neurophysiol 101, 1016-1032.

Forkink M., Smeitink J. A., Brock R., Willems P. H. and Koopman W. J. (2010) Detection and manipulation of mitochondrial reactive oxygen species in mammalian cells. Biochim Biophys Acta 1797, 1034-1044.

Formichi P., Battisti C., Dotti M. T., Hayek G., Zappella M. and Federico A. (1998) Vitamin E serum levels in Rett syndrome. J Neurol Sci 156, 227-230.

Forte M., Gold B. G., Marracci G., Chaudhary P., Basso E., Johnsen D., Yu X., Fowlkes J., Rahder M., Stem K., Bernardi P. and Bourdette D. (2007) Cyclophilin $\mathrm{D}$ inactivation protects axons in experimental autoimmune encephalomyelitis, an animal model of multiple sclerosis. Proc Natl Acad Sci U S A 104, 7558-7563.

Foster K. A., Galeffi F., Gerich F. J., Turner D. A. and Müller M. (2006) Optical and pharmacological tools to investigate the role of mitochondria during oxidative stress and neurodegeneration. Prog Neurobiol 79, 136-171. 
Funke F., Gerich F. J. and Müller M. (2011) Dynamic, semi-quantitative imaging of intracellular ROS levels and redox status in rat hippocampal neurons. Neuroimage 54, 2590-2602.

Gallop P. M., Paz M. A., Henson E. and Latt S. A. (1984) Dynamic approaches to the delivery of reporter reagents into living cells. BioTechniques 2, 32-36.

Galvao T. C. and Thomas J. O. (2005) Structure-specific binding of MeCP2 to four-way junction DNA through its methyl CpG-binding domain. Nucleic Acids Res 33, 6603-6609.

Gerich F. J., Funke F., Hildebrandt B., Faßhauer M. and Müller M. (2009) $\mathrm{H}_{2} \mathrm{O}_{2}$-mediated modulation of cytosolic signaling and organelle function in rat hippocampus. Pflügers Arch 458, 937-952.

Gibson J. H., Slobedman B., K N. H., Williamson S. L., Minchenko D., El-Osta A., Stern J. L. and Christodoulou J. (2010) Downstream targets of methyl CpG binding protein 2 and their abnormal expression in the frontal cortex of the human Rett syndrome brain. BMC Neurosci 11, 53.

Gomez-Isla T., Spires T., De Calignon A. and Hyman B. T. (2008) Neuropathology of Alzheimer's disease. Handb Clin Neurol 89, 233-243.

Graham D. G. (1978) Oxidative pathways for catecholamines in the genesis of neuromelanin and cytotoxic quinones. Mol Pharmacol 14, 633-643.

Greenwood S. M., Mizielinska S. M., Frenguelli B. G., Harvey J. and Connolly C. N. (2007) Mitochondrial dysfunction and dendritic beading during neuronal toxicity. J Biol Chem 282, 26235-26244.

Großer E., Hirt U., Janc O. A., Menzfeld C., Fischer M., Kempkes B., Vogelgesang S., Manzke T. U., Opitz L., Salinas-Riester G. and Müller M. (2012) Oxidative burden and mitochondrial dysfunction in a mouse model of Rett syndrome. Neurobiol Dis 48, 102-114.

Grynkiewicz G., Poenie M. and Tsien R. Y. (1985) A new generation of $\mathrm{Ca}^{2+}$ indicators with greatly improved fluorescence properties. J Biol Chem 260, 3440-3450.

Gutscher M., Pauleau A. L., Marty L., Brach T., Wabnitz G. H., Samstag Y., Meyer A. J. and Dick T. P. (2008) Real-time imaging of the intracellular glutathione redox potential. Nat Methods 5, 553-559.

Guy J., Hendrich B., Holmes M., Martin J. E. and Bird A. (2001) A mouse Mecp2-null mutation causes neurological symptoms that mimic Rett syndrome. Nat Genet 27, 322-326.

Guzy R. D., Hoyos B., Robin E., Chen H., Liu L., Mansfield K. D., Simon M. C., Hammerling U. and Schumacker P. T. (2005) Mitochondrial complex III is required for hypoxia-induced ROS production and cellular oxygen sensing. Cell Metab 1, 401-408. 
Ha J. S., Lee C. S., Maeng J. S., Kwon K. S. and Park S. S. (2009) Chronic glutamate toxicity in mouse cortical neuron culture. Brain Res 1273, 138-143.

Hagberg B. (2005) Rett syndrome: long-term clinical follow-up experiences over four decades. J Child Neurol 20, 722-727.

Hagberg B., Berg M. and Steffenburg U. (2001) Three decades of sociomedical experiences from West Swedish Rett females 4-60 years of age. Brain Dev 23 Suppl 1, S28-31.

Hagberg B., Aicardi J., Dias K. and Ramos O. (1983) A progressive syndrome of autism, dementia, ataxia, and loss of purposeful hand use in girls: Rett's syndrome: report of 35 cases. Ann Neurol 14, 471-479.

Halliwell B. and Gutteridge J. M. (1984) Oxygen toxicity, oxygen radicals, transition metals and disease. Biochem $J$ 219, 1-14.

Halliwell B. and Cross C. E. (1994) Oxygen-derived species: their relation to human disease and environmental stress. Environ Health Perspect 102 Suppl 10, 5-12.

Hamberger A., Gillberg C., Palm A. and Hagberg B. (1992) Elevated CSF glutamate in Rett syndrome. Neuropediatrics 23, 212-213.

Hanson G. T., Aggeler R., Oglesbee D., Cannon M., Capaldi R. A., Tsien R. Y. and Remington S. J. (2004) Investigating mitochondrial redox potential with redox-sensitive green fluorescent protein indicators. J Biol Chem 279, 1304413053.

Harman D. (2003) The free radical theory of aging. Antioxid Redox Signal 5, 557-561.

Hasel P., McKay S., Qiu J. and Hardingham G. E. (2015) Selective dendritic susceptibility to bioenergetic, excitotoxic and redox perturbations in cortical neurons. Biochim Biophys Acta 1853, 2066-2076.

Heck S., Lezoualc'h F., Engert S. and Behl C. (1999) Insulin-like growth factor-1-mediated neuroprotection against oxidative stress is associated with activation of nuclear factor kappaB. J Biol Chem 274, 9828-9835.

Hendrich B. and Bird A. (1998) Identification and characterization of a family of mammalian methyl-CpG binding proteins. Mol Cell Biol 18, 6538-6547.

Hongpaisan J., Winters C. A. and Andrews S. B. (2004) Strong calcium entry activates mitochondrial superoxide generation, upregulating kinase signaling in hippocampal neurons. J Neurosci 24, 10878-10887.

Jahn U., Galano J. M. and Durand T. (2008) Beyond prostaglandins-chemistry and biology of cyclic oxygenated metabolites formed by free-radical pathways from polyunsaturated fatty acids. Angew Chem Int Ed Engl 47, 5894-5955. 
Jellinger K., Armstrong D., Zoghbi H. Y. and Percy A. K. (1988) Neuropathology of Rett syndrome. Acta Neuropathol 76, 142-158.

Jellinger K. A. (2003) Rett Syndrome -- an update. J Neural Transm (Vienna) 110, 681-701.

Jentarra G. M., Olfers S. L., Rice S. G., Srivastava N., Homanics G. E., Blue M., Naidu S. and Narayanan V. (2010) Abnormalities of cell packing density and dendritic complexity in the MeCP2 A140V mouse model of Rett syndrome/X-linked mental retardation. BMC Neurosci 11, 19.

Julu P. O., Kerr A. M., Apartopoulos F., Al-Rawas S., Engerström I. W., Engerström L., Jamal G. A. and Hansen S. (2001) Characterisation of breathing and associated central autonomic dysfunction in the Rett disorder. Arch Dis Child 85, 29-37.

Kadiiska M. B., Gladen B. C., Baird D. D., Graham L. B., Parker C. E., Ames B. N., Basu S., Fitzgerald G. A., Lawson J. A., Marnett L. J., Morrow J. D., Murray D. M., Plastaras J., Roberts L. J., 2nd, Rokach J., Shigenaga M. K., Sun J., Walter P. B., Tomer K. B., Barrett J. C. and Mason R. P. (2005) Biomarkers of oxidative stress study III. Effects of the nonsteroidal antiinflammatory agents indomethacin and meclofenamic acid on measurements of oxidative products of lipids in $\mathrm{CCl} 4$ poisoning. Free Radic Biol Med 38, 711-718.

Kamsler A. and Segal M. (2003) Paradoxical actions of hydrogen peroxide on long-term potentiation in transgenic superoxide dismutase-1 mice. $J$ Neurosci 23, 10359-10367.

Katz D. M., Dutschmann M., Ramirez J. M. and Hilaire G. (2009) Breathing disorders in Rett syndrome: progressive neurochemical dysfunction in the respiratory network after birth. Respir Physiol Neurobiol 168, 101-108.

Keller G. A., Warner T. G., Steimer K. S. and Hallewell R. A. (1991) Cu,Zn superoxide dismutase is a peroxisomal enzyme in human fibroblasts and hepatoma cells. Proc Natl Acad Sci U S A 88, 7381-7385.

Kerr A. M., Armstrong D. D., Prescott R. J., Doyle D. and Kearney D. L. (1997) Rett syndrome: analysis of deaths in the British survey. Eur Child Adolesc Psychiatry 6 Suppl 1, 71-74.

Kishi N. and Macklis J. D. (2004) MECP2 is progressively expressed in postmigratory neurons and is involved in neuronal maturation rather than cell fate decisions. Mol Cell Neurosci 27, 306-321.

Klose R. J., Sarraf S. A., Schmiedeberg L., McDermott S. M., Stancheva I. and Bird A. P. (2005) DNA binding selectivity of MeCP2 due to a requirement for A/T sequences adjacent to methyl-CpG. Mol Cell 19, 667-678.

Kolossov V. L., Spring B. Q., Sokolowski A., Conour J. E., Clegg R. M., Kenis P. J. and Gaskins H. R. (2008) Engineering redox-sensitive linkers for genetically encoded FRET-based biosensors. Exp Biol Med 233, 238-248. 
Kovac S., Domijan A. M., Walker M. C. and Abramov A. Y. (2014) Seizure activity results in calcium- and mitochondria-independent ROS production via NADPH and xanthine oxidase activation. Cell Death Dis 5, e1442.

Kratschmar D. V., Calabrese D., Walsh J., Lister A., Birk J., AppenzellerHerzog C., Moulin P., Goldring C. E. and Odermatt A. (2012) Suppression of the Nrf2-dependent antioxidant response by glucocorticoids and 11betaHSD1-mediated glucocorticoid activation in hepatic cells. PLOS ONE 7, e36774.

Kriaucionis S., Paterson A., Curtis J., Guy J., Macleod N. and Bird A. (2006) Gene expression analysis exposes mitochondrial abnormalities in a mouse model of Rett syndrome. Mol Cell Biol 26, 5033-5042.

Kron M. and Müller M. (2010) Impaired hippocampal $\mathrm{Ca}^{2+}$ homeostasis and concomitant $\mathrm{K}^{+}$channel dysfunction in a mouse model of Rett syndrome during anoxia. Neuroscience 171, 300-315.

Kron M., Zimmermann J. L., Dutschmann M., Funke F. and Müller M. (2011) Altered responses of MeCP2-deficient mouse brain stem to severe hypoxia. $J$ Neurophysiol 105, 3067-3079.

Kroncke K. D. (2003) Nitrosative stress and transcription. Biol Chem 384, 1365-1377.

Lappalainen R. and Riikonen R. S. (1994) Elevated CSF lactate in the Rett syndrome: cause or consequence? Brain Dev 16, 399-401.

Lappalainen R. and Riikonen R. S. (1996) High levels of cerebrospinal fluid glutamate in Rett syndrome. Pediatr Neurol 15, 213-216.

Lappalainen R., Lindholm D. and Riikonen R. (1996) Low levels of nerve growth factor in cerebrospinal fluid of children with Rett syndrome. J Child Neurol 11, 296-300.

Lawson-Yuen A., Liu D., Han L., Jiang Z. I., Tsai G. E., Basu A. C., Picker J., Feng J. and Coyle J. T. (2007) Ube3a mRNA and protein expression are not decreased in Mecp2R168X mutant mice. Brain Res 1180, 1-6.

LeBel C. P., Ischiropoulos H. and Bondy S. C. (1992) Evaluation of the probe 2',7'-dichlorofluorescin as an indicator of reactive oxygen species formation and oxidative stress. Chem Res Toxicol 5, 227-231.

Lee B., Cao R., Choi Y. S., Cho H. Y., Rhee A. D., Hah C. K., Hoyt K. R. and Obrietan K. (2009) The CREB/CRE transcriptional pathway: protection against oxidative stress-mediated neuronal cell death. J Neurochem 108, 1251-1265.

Lee C. W. and Peng H. B. (2006) Mitochondrial clustering at the vertebrate neuromuscular junction during presynaptic differentiation. J Neurobiol 66, 522-536. 
Lee D., Huang W., Wang L., Copolov D. and Lim A. T. (2000) Glucocorticoid modulation of dopamine mediated effects on hypothalamic atrial natriuretic factor neurons. Mol Psychiatry 5, 332-336.

Lekman A., Witt-Engerström I., Holmberg B., Percy A., Svennerholm L. and Hagberg B. (1990) CSF and urine biogenic amine metabolites in Rett syndrome. Clin Genet 37, 173-178.

Li Y., Wang H., Muffat J., Cheng A. W., Orlando D. A., Loven J., Kwok S. M., Feldman D. A., Bateup H. S., Gao Q., Hockemeyer D., Mitalipova M., Lewis C. A., Vander Heiden M. G., Sur M., Young R. A. and Jaenisch R. (2013) Global transcriptional and translational repression in human-embryonic-stemcell-derived Rett syndrome neurons. Cell Stem Cell 13, 446-458.

Liou W., Chang L. Y., Geuze H. J., Strous G. J., Crapo J. D. and Slot J. W. (1993) Distribution of CuZn superoxide dismutase in rat liver. Free Radic Biol Med 14, 201-207.

Lipton P. (1999) Ischemic cell death in brain neurons. Physiol Rev 79, 1431 1568.

Lipton S. A. and Nicotera P. (1998) Calcium, free radicals and excitotoxins in neuronal apoptosis. Cell Calcium 23, 165-171.

Liu K., Nagase H., Huang C. G., Calamita G. and Agre P. (2006) Purification and functional characterization of aquaporin-8. Biol Cell 98, 153-161.

Loschen G., Flohe L. and Chance B. (1971) Respiratory chain linked $\mathrm{H}_{2} \mathrm{O}_{2}$ production in pigeon heart mitochondria. FEBS Lett 18, 261-264.

Luck H. (1954) Quantitative determination of catalase activity of biological material. Enzymologia 17, 31-40.

Maezawa I. and Jin L. W. (2010) Rett syndrome microglia damage dendrites and synapses by the elevated release of glutamate. $J$ Neurosci 30, 53465356.

Malinouski M., Zhou Y., Belousov V. V., Hatfield D. L. and Gladyshev V. N. (2011) Hydrogen peroxide probes directed to different cellular compartments. PLOS ONE 6, e14564.

Marino S., Marani L., Nazzaro C., Beani L. and Siniscalchi A. (2007) Mechanisms of sodium azide-induced changes in intracellular calcium concentration in rat primary cortical neurons. Neurotoxicology 28, 622-629.

Markvicheva K. N., Bogdanova E. A., Staroverov D. B., Lukyanov S. and Belousov V. V. (2008) Imaging of intracellular hydrogen peroxide production with HyPer upon stimulation of HeLa cells with epidermal growth factor. Methods Mol Biol 476, 79-86. 
Matsuishi T., Yamashita Y., Takahashi T. and Nagamitsu S. (2011) Rett syndrome: the state of clinical and basic research, and future perspectives. Brain Dev 33, 627-631.

Matsuishi T., Urabe F., Komori H., Yamashita Y., Naito E., Kuroda Y., Horikawa M. and Ohtaki E. (1992) The Rett syndrome and CSF lactic acid patterns. Brain Dev 14, 68-70.

Matsuishi T., Nagamitsu S., Yamashita Y., Murakami Y., Kimura A., Sakai T., Shoji H., Kato H. and Percy A. K. (1997) Decreased cerebrospinal fluid levels of substance P in patients with Rett syndrome. Ann Neurol 42, 978-981.

Mattson M. P. (2007) Mitochondrial regulation of neuronal plasticity. Neurochem Res 32, 707-715.

Mattson M. P. and Partin J. (1999) Evidence for mitochondrial control of neuronal polarity. J Neurosci Res 56, 8-20.

Meldrum B. S. (2000) Glutamate as a neurotransmitter in the brain: review of physiology and pathology. J Nutr 130, 1007S-1015S.

Meloni I., Bruttini M., Longo I., Mari F., Rizzolio F., D'Adamo P., Denvriendt K., Fryns J. P., Toniolo D. and Renieri A. (2000) A mutation in the rett syndrome gene, MECP2, causes $X$-linked mental retardation and progressive spasticity in males. Am J Hum Genet 67, 982-985.

Menzfeld C., Janc O. A., Dudek J., Rehling P. and Müller M. (2014) Mitochondrial dysfunction in Rett syndrome viewed from the biochemical perspective. 13th annual Rett syndrome symposium, Chantilly, VA, Abstract p.78.

Michaels R. L. and Rothman S. M. (1990) Glutamate neurotoxicity in vitro: antagonist pharmacology and intracellular calcium concentrations. J Neurosci 10, 283-292.

Milne G. L., Yin H., Hardy K. D., Davies S. S. and Roberts L. J., 2nd (2011) Isoprostane generation and function. Chem Rev 111, 5973-5996.

Milton V. J. and Sweeney S. T. (2011) Oxidative stress in synapse development and function. Dev Neurobiol 72, 100-110.

Mironov S. L., Skorova E., Hartelt N., Mironova L. A., Hasan M. T. and Kügler S. (2009) Remodelling of the respiratory network in a mouse model of Rett syndrome depends on brain-derived neurotrophic factor regulated slow calcium buffering. J Physiol 587, 2473-2485.

Miyazaki I. and Asanuma M. (2008) Dopaminergic neuron-specific oxidative stress caused by dopamine itself. Acta Med Okayama 62, 141-150.

Mohanty J. G., Jaffe J. S., Schulman E. S. and Raible D. G. (1997) A highly sensitive fluorescent micro-assay of $\mathrm{H}_{2} \mathrm{O}_{2}$ release from activated human 
leukocytes using a dihydroxyphenoxazine derivative. $J$ Immunol Methods 202, 133-141.

Mullaney B. C., Johnston M. V. and Blue M. E. (2004) Developmental expression of methyl-CpG binding protein 2 is dynamically regulated in the rodent brain. Neuroscience 123, 939-949.

Müller M. and Can K. (2014) Aberrant redox homoeostasis and mitochondrial dysfunction in Rett syndrome. Biochem Soc Trans 42, 959-964.

Nan X., Campoy F. J. and Bird A. (1997) MeCP2 is a transcriptional repressor with abundant binding sites in genomic chromatin. Cell 88, 471-481.

Nathan C. (2003) Specificity of a third kind: reactive oxygen and nitrogen intermediates in cell signaling. J Clin Invest 111, 769-778.

Nicholls D. G. and Budd S. L. (2000) Mitochondria and neuronal survival. Physiol Rev 80, 315-360.

Ogier M. and Katz D. M. (2008) Breathing dysfunction in Rett syndrome: understanding epigenetic regulation of the respiratory network. Respir Physiol Neurobiol 164, 55-63.

Olney J. W. (1969) Brain lesions, obesity, and other disturbances in mice treated with monosodium glutamate. Science 164, 719-721.

Orrico A., Lam C., Galli L., Dotti M. T., Hayek G., Tong S. F., Poon P. M., Zappella M., Federico A. and Sorrentino V. (2000) MECP2 mutation in male patients with non-specific X-linked mental retardation. FEBS Lett 481, 285288.

Ostergaard H., Henriksen A., Hansen F. G. and Winther J. R. (2001) Shedding light on disulfide bond formation: engineering a redox switch in green fluorescent protein. Embo J 20, 5853-5862.

Pecorelli A., Leoni G., Cervellati F., Canali R., Signorini C., Leoncini S., Cortelazzo A., De Felice C., Ciccoli L., Hayek J. and Valacchi G. (2013) Genes related to mitochondrial functions, protein degradation, and chromatin folding are differentially expressed in lymphomonocytes of Rett syndrome patients. Mediators Inflamm 2013, 137629.

Pelka G. J., Watson C. M., Christodoulou J. and Tam P. P. (2005) Distinct expression profiles of Mecp2 transcripts with different lengths of 3'UTR in the brain and visceral organs during mouse development. Genomics 85, 441-452.

Pelka G. J., Watson C. M., Radziewic T., Hayward M., Lahooti H., Christodoulou J. and Tam P. P. (2006) Mecp2 deficiency is associated with learning and cognitive deficits and altered gene activity in the hippocampal region of mice. Brain 129, 887-898.

Percy A. K. (1992) Neurochemistry of the Rett syndrome. Brain Dev 14 Suppl, S57-62. 
Pereira L. O., Nabinger P. M., Strapasson A. C., Nardin P., Goncalves C. A., Siqueira I. R. and Netto C. A. (2009) Long-term effects of environmental stimulation following hypoxia-ischemia on the oxidative state and BDNF levels in rat hippocampus and frontal cortex. Brain Res 1247, 188-195.

Pin J. P. and Duvoisin R. (1995) The metabotropic glutamate receptors: structure and functions. Neuropharmacology 34, 1-26.

Pletjushkina O. Y., Lyamzaev K. G., Popova E. N., Nepryakhina O. K., Ivanova O. Y., Domnina L. V., Chernyak B. V. and Skulachev V. P. (2006) Effect of oxidative stress on dynamics of mitochondrial reticulum. Biochim Biophys Acta 1757, 518-524.

Poburko D., Santo-Domingo J. and Demaurex N. (2011) Dynamic regulation of the mitochondrial proton gradient during cytosolic calcium elevations. J Biol Chem 286, 11672-11684.

Pulsinelli W. A., Brierley J. B. and Plum F. (1982) Temporal profile of neuronal damage in a model of transient forebrain ischemia. Ann Neurol 11, 491-498.

Rahman T., Hosen I., Islam M. M. T. and Shekhar H. U. (2012) Oxidative Stress and Human Health. Adv Biosci Biotechnol 3, 997-1019.

Reiss A. L., Faruque F., Naidu S., Abrams M., Beaty T., Bryan R. N. and Moser H. (1993) Neuroanatomy of Rett syndrome: a volumetric imaging study. Ann Neurol 34, 227-234.

Rett A. (1966) Über ein eigenartiges hirnatrophisches Syndrom bei Hyperammonämie im Kindesalter. Wien Med Wochenschr 116, 723-726.

Ricceri L., De Filippis B. and Laviola G. (2008) Mouse models of Rett syndrome: from behavioural phenotyping to preclinical evaluation of new therapeutic approaches. Behav Pharmacol 19, 501-517.

Riikonen R. and Vanhala R. (1999) Levels of cerebrospinal fluid nerve-growth factor differ in infantile autism and Rett syndrome. Dev Med Child Neurol 41, 148-152.

Robinson K. M., Janes M. S., Pehar M., Monette J. S., Ross M. F., Hagen T. M., Murphy M. P. and Beckman J. S. (2006) Selective fluorescent imaging of superoxide in vivo using ethidium-based probes. Proc Natl Acad Sci US A 103, 15038-15043.

Rocher A. B., Kinson M. S. and Luebke J. I. (2008) Significant structural but not physiological changes in cortical neurons of 12-month-old Tg2576 mice. Neurobiol Dis 32, 309-318.

Roma L. P., Duprez J., Takahashi H. K., Gilon P., Wiederkehr A. and Jonas J. C. (2012) Dynamic measurements of mitochondrial hydrogen peroxide concentration and glutathione redox state in rat pancreatic beta-cells using 
ratiometric fluorescent proteins: confounding effects of $\mathrm{pH}$ with HyPer but not roGFP1. Biochem J 441, 971-978.

Ross M. A. (2000) Could oxidative stress be a factor in neurodevelopmental disorders? Prostaglandins Leukot Essent Fatty Acids 63, 61-63.

Ruch A., Kurczynski T. W. and Velasco M. E. (1989) Mitochondrial alterations in Rett syndrome. Pediatr Neurol 5, 320-323.

Saywell V., Viola A., Confort-Gouny S., Le Fur Y., Villard L. and Cozzone P. J. (2006) Brain magnetic resonance study of Mecp2 deletion effects on anatomy and metabolism. Biochem Biophys Res Commun 340, 776-783.

Schanen N. C., Dahle E. J., Capozzoli F., Holm V. A., Zoghbi H. Y. and Francke U. (1997) A new Rett syndrome family consistent with X-linked inheritance expands the X chromosome exclusion map. Am J Hum Genet 61, 634-641.

Schlue W. R. and Thomas R. C. (1985) A dual mechanism for intracellular pH regulation by leech neurones. J Physiol 364, 327-338.

Schmidt-Kastner R. and Freund T. F. (1991) Selective vulnerability of the hippocampus in brain ischemia. Neuroscience 40, 599-636.

Schoepp D. D. and Conn P. J. (1993) Metabotropic glutamate receptors in brain function and pathology. Trends Pharmacol Sci 14, 13-20.

Schultz R. J., Glaze D. G., Motil K. J., Armstrong D. D., del Junco D. J., Hubbard C. R. and Percy A. K. (1993) The pattern of growth failure in Rett syndrome. Am J Dis Child 147, 633-637.

Schwarzländer M., Logan D. C., Fricker M. D. and Sweetlove L. J. (2011) The circularly permuted yellow fluorescent protein cpYFP that has been used as a superoxide probe is highly responsive to $\mathrm{pH}$ but not superoxide in mitochondria: implications for the existence of superoxide 'flashes'. Biochem $J$ 437, 381-387.

Segawa M. (1997) Pathophysiology of Rett syndrome from the standpoint of early catecholamine disturbance. Eur Child Adolesc Psychiatry 6 Suppl 1, 5660.

Shahbazian M., Young J., Yuva-Paylor L., Spencer C., Antalffy B., Noebels J., Armstrong D., Paylor R. and Zoghbi H. (2002) Mice with truncated MeCP2 recapitulate many Rett syndrome features and display hyperacetylation of histone H3. Neuron 35, 243-254.

Shahbazian M. D., Antalffy B., Armstrong D. L. and Zoghbi H. Y. (2002) Insight into Rett syndrome: MeCP2 levels display tissue- and cell-specific differences and correlate with neuronal maturation. Hum Mol Genet 11, 115124. 
Sharp A. P. and Thomas R. C. (1981) The effects of chloride substitution on intracellular $\mathrm{pH}$ in crab muscle. J Physio/ 312, 71-80.

Sierra C., Vilaseca M. A., Brandi N., Artuch R., Mira A., Nieto M. and Pineda M. (2001) Oxidative stress in Rett syndrome. Brain Dev 23 Suppl 1, S236239.

Sies H. (1991) Oxidative stress: from basic research to clinical application. Am J Med 91, 31S-38S.

Simonian N. A. and Coyle J. T. (1996) Oxidative stress in neurodegenerative diseases. Annu Rev Pharmacol Toxicol 36, 83-106.

Sinha K., Das J., Pal P. B. and Sil P. C. Oxidative stress: the mitochondriadependent and mitochondria-independent pathways of apoptosis. Arch Toxicol 87, 1157-1180.

Sinha K., Das J., Pal P. B. and Sil P. C. (2013) Oxidative stress: the mitochondria-dependent and mitochondria-independent pathways of apoptosis. Arch Toxicol 87, 1157-1180.

Siskova Z., Justus D., Kaneko H., Friedrichs D., Henneberg N., Beutel T., Pitsch J., Schoch S., Becker A., von der Kammer H. and Remy S. (2014) Dendritic structural degeneration is functionally linked to cellular hyperexcitability in a mouse model of Alzheimer's disease. Neuron 84, 10231033.

Sofić E., Riederer P., Killian W. and Rett A. (1987) Reduced concentrations of ascorbic acid and glutathione in a single case of Rett syndrome: a postmortem brain study. Brain Dev 9, 529-531.

Stamer K., Vogel R., Thies E., Mandelkow E. and Mandelkow E. M. (2002) Tau blocks traffic of organelles, neurofilaments, and APP vesicles in neurons and enhances oxidative stress. J Cell Biol 156, 1051-1063.

Stettner G. M., Huppke P., Gärtner J., Richter D. W. and Dutschmann M. (2008) Disturbances of breathing in Rett syndrome: Results from patients and animal models. Adv Exp Biol Med 605, 503-507.

Sudha K., Rao A. V. and Rao A. (2001) Oxidative stress and antioxidants in epilepsy. Clin Chim Acta 303, 19-24.

Sulzer D., Bogulavsky J., Larsen K. E., Behr G., Karatekin E., Kleinman M. H., Turro N., Krantz D., Edwards R. H., Greene L. A. and Zecca L. (2000) Neuromelanin biosynthesis is driven by excess cytosolic catecholamines not accumulated by synaptic vesicles. Proc Natl Acad Sci U S A 97, 1186911874.

Tao J., Wu H. and Sun Y. E. (2009) Deciphering Rett syndrome with mouse genetics, epigenomics, and human neurons. Int Rev Neurobio/ 89, 147-160. 
Tao J., Van Esch H., Hagedorn-Greiwe M., Hoffmann K., Moser B., Raynaud M., Sperner J., Fryns J. P., Schwinger E., Gecz J., Ropers H. H. and Kalscheuer V. M. (2004) Mutations in the X-linked cyclin-dependent kinaselike 5 (CDKL5/STK9) gene are associated with severe neurodevelopmental retardation. Am J Hum Genet 75, 1149-1154.

Toloe J., Mollajew R., Kügler S. and Mironov S. L. (2014) Metabolic differences in hippocampal 'Rett' neurons revealed by ATP imaging. Mol Cell Neurosci 59C, 47-56.

Trappe R., Laccone F., Cobilanschi J., Meins M., Huppke P., Hanefeld F. and Engel W. (2001) MECP2 mutations in sporadic cases of Rett syndrome are almost exclusively of paternal origin. Am J Hum Genet 68, 1093-1101.

Tropea D., Giacometti E., Wilson N. R., Beard C., McCurry C., Fu D. D., Flannery R., Jaenisch R. and Sur M. (2009) Partial reversal of Rett Syndrome-like symptoms in MeCP2 mutant mice. Proc Natl Acad Sci U S A 106, 2029-2034.

Valinluck V., Tsai H. H., Rogstad D. K., Burdzy A., Bird A. and Sowers L. C. (2004) Oxidative damage to methyl-CpG sequences inhibits the binding of the methyl-CpG binding domain (MBD) of methyl-CpG binding protein 2 (MeCP2). Nucleic Acids Res 32, 4100-4108.

Vayssiere J. L., Cordeau-Lossouarn L., Larcher J. C., Basseville M., Gros F. and Croizat B. (1992) Participation of the mitochondrial genome in the differentiation of neuroblastoma cells. In Vitro Cell Dev Biol 28A, 763-772.

Viemari J. C., Roux J. C., Tryba A. K., Saywell V., Burnet H., Pena F., Zanella S., Bevengut M., Barthelemy-Requin M., Herzing L. B., Moncla A., Mancini J., Ramirez J. M., Villard L. and Hilaire G. (2005) Mecp2 deficiency disrupts norepinephrine and respiratory systems in mice. J Neurosci 25, 11521-11530.

Villard L., Kpebe A., Cardoso C., Chelly P. J., Tardieu P. M. and Fontes M. (2000) Two affected boys in a Rett syndrome family: clinical and molecular findings. Neurology 55, 1188-1193.

Wachter R. M., Yarbrough D., Kallio K. and Remington S. J. (2000) Crystallographic and energetic analysis of binding of selected anions to the yellow variants of green fluorescent protein. J Mol Biol 301, 157-171.

Wagener K. C., Kolbrink B., Dietrich K., Kizina K. M., Terwitte L. S., Kempkes B., Bao G. and Müller M. (2016) Redox-indicator mice stably expressing genetically-encoded neuronal roGFP: Versatile tools to decipher subcellular redox dynamics in neuropathophysiology. Antioxid Redox Signal in press, doi:10.1089/ars.2015.6587.

Wan M., Lee S. S., Zhang X., Houwink-Manville I., Song H. R., Amir R. E., Budden S., Naidu S., Pereira J. L., Lo I. F., Zoghbi H. Y., Schanen N. C. and Francke U. (1999) Rett syndrome and beyond: recurrent spontaneous and familial MECP2 mutations at CpG hotspots. Am J Hum Genet 65, 1520-1529. 
Wang X., Pal R., Chen X. W., Kumar K. N., Kim O. J. and Michaelis E. K. (2007) Genome-wide transcriptome profiling of region-specific vulnerability to oxidative stress in the hippocampus. Genomics 90, 201-212.

Weaving L. S., Ellaway C. J., Gecz J. and Christodoulou J. (2005) Rett syndrome: clinical review and genetic update. J Med Genet 42, 1-7.

Weaving L. S., Christodoulou J., Williamson S. L., Friend K. L., McKenzie O. L., Archer H., Evans J., Clarke A., Pelka G. J., Tam P. P., Watson C., Lahooti H., Ellaway C. J., Bennetts B., Leonard H. and Gecz J. (2004) Mutations of CDKL5 cause a severe neurodevelopmental disorder with infantile spasms and mental retardation. Am J Hum Genet 75, 1079-1093.

Wegener E., Brendel C., Fischer A., Hulsmann S., Gartner J. and Huppke P. (2014) Characterization of the MeCP2R168X Knockin Mouse Model for Rett Syndrome. PLoS One 9, e115444.

Weisiger R. A. and Fridovich I. (1973) Mitochondrial superoxide simutase. Site of synthesis and intramitochondrial localization. J Biol Chem 248, 47934796.

Weitzman S. A., Turk P. W., Milkowski D. H. and Kozlowski K. (1994) Free radical adducts induce alterations in DNA cytosine methylation. Proc Natl Acad Sci U S A 91, 1261-1264.

Weller J., Kizina K. M., Can K., Bao G. and Muller M. (2014) Response properties of the genetically encoded optical $\mathrm{HO}$ sensor HyPer. Free Radic Biol Med 76C, 227-241.

Weng S. M., Bailey M. E. and Cobb S. R. (2011) Rett syndrome: from bed to bench. Pediatr Neonatol 52, 309-316.

Wenk G. L. (1997) Rett syndrome: neurobiological changes underlying specific symptoms. Prog Neurobiol 51, 383-391.

Wenk G. L. and Mobley S. L. (1996) Choline acetyltransferase activity and vesamicol binding in Rett syndrome and in rats with nucleus basalis lesions. Neuroscience 73, 79-84.

Wenk G. L. and Hauss-Wegrzyniak B. (1999) Altered cholinergic function in the basal forebrain of girls with Rett syndrome. Neuropediatrics 30, 125-129.

Weyemi U., Lagente-Chevallier O., Boufraqech M., Prenois F., Courtin F., Caillou B., Talbot M., Dardalhon M., Al Ghuzlan A., Bidart J. M., Schlumberger M. and Dupuy C. (2012) ROS-generating NADPH oxidase NOX4 is a critical mediator in oncogenic H-Ras-induced DNA damage and subsequent senescence. Oncogene 31, 1117-1129.

Wilde G. J., Pringle A. K., Wright P. and lannotti F. (1997) Differential vulnerability of the CA1 and CA3 subfields of the hippocampus to superoxide and hydroxyl radicals in vitro. $J$ Neurochem $69,883-886$. 
Wrona M. Z., Yang Z., Zhang F. and Dryhurst G. (1997) Potential new insights into the molecular mechanisms of methamphetamine-induced neurodegeneration. NIDA Res Monogr 173, 146-174.

Yano T., Oku M., Akeyama N., Itoyama A., Yurimoto H., Kuge S., Fujiki Y. and Sakai Y. (2010) A novel fluorescent sensor protein for visualization of redox states in the cytoplasm and in peroxisomes. Mol Cell Biol 30, 37583766.

Young D., Nagarajan L., de Klerk N., Jacoby P., Ellaway C. and Leonard H. (2007) Sleep problems in Rett syndrome. Brain Dev 29, 609-616.

Young N., Mikhalkevich N., Yan Y., Chen D. and Zheng W. P. (2005) Differential regulation of osteoblast activity by Th cell subsets mediated by parathyroid hormone and IFN-gamma. J Immunol 175, 8287-8295.

Zelko I. N., Mariani T. J. and Folz R. J. (2002) Superoxide dismutase multigene family: a comparison of the CuZn-SOD (SOD1), Mn-SOD (SOD2), and EC-SOD (SOD3) gene structures, evolution, and expression. Free Radic Biol Med 33, 337-349.

Zhang J., Perry G., Smith M. A., Robertson D., Olson S. J., Graham D. G. and Montine T. J. (1999) Parkinson's disease is associated with oxidative damage to cytoplasmic DNA and RNA in substantia nigra neurons. Am J Pathol 154, 1423-1429.

Zoghbi H. (1988) Genetic aspects of Rett syndrome. J Child Neurol 3 Suppl, S76-78.

Zoghbi H. Y., Percy A. K., Glaze D. G., Butler I. J. and Riccardi V. M. (1985) Reduction of biogenic amine levels in the Rett syndrome. N Engl J Med 313, 921-924.

Zoghbi H. Y., Milstien S., Butler I. J., Smith E. O., Kaufman S., Glaze D. G. and Percy A. K. (1989) Cerebrospinal fluid biogenic amines and biopterin in Rett syndrome. Ann Neurol 25, 56-60. 


\section{Acknowledgements}

I would like to express sincere gratitude to my supervisor Prof. Michael Müller for giving me the opportunity to work in his lab. I am gratefully thankful for his outstanding guidance, generous support, great leadership, good advice and critics.

I greatly appreciate my thesis committee members Prof. Detlev Schild and Dr. Sebastian Kügler for insightful discussions, advice and helpful suggestions to my project. I also thank Dr. Sebastian Kügler and Dr. Johan Tolö for their collaboration in order to make the viral vector available for my thesis.

I am very grateful to Prof. S. James Remington for making available to our group the roGFP coding plasmids.

I sincerely thank the DFG CNMPB for accepting my project to the CNMPB program.

I would like to truly thank Prof. Swen Hülsmann for his good advice and stimulating discussions about the project.

My special appreciation goes to Axel Zigan for his commitment in taking care of my mice. A big thank you goes also to all the people from the mechanical workshop.

I would like to gratefully acknowledge my friends and colleagues. Especially, I thank Belinda Kempkes for the best technical assistance, her abundant help during my whole work and for spreading her unique positive attitude in our lab ;) Furthermore, I would like to express my deepest thanks to my close friends Dr. Oliwia Janc, Dr. Christiane Menzfeld and Dr. Chiranjeevi Bodda for their precious time spent on valuable discussions and advice. Thank you all for your priceless support, patience and for your presence during my moments of weakness. Thank you that you have never doubted me. I will always appreciate your motivation and encouragement. My warm thanks go also to 
Dr. Katharina Dietrich and Lukas Terwitte for helping me with slicing and taking images. Moreover, I thank Anja-Annett Grützner, Kerstin Wagener, Mark Hüser as well as all former students of MM and Swen Hülsmann's groups for their great company in the lab.

Finally, the absolutely biggest thank you I would like to address to people closest to my heart: Teresa Can, Jerzy Can and Katarzyna Can. Kochana Mamo, Kochany Tato, Kochana Siostro, dziękuję Wam za to, że nigdy we mnie nie zwątpiliście. Dziękuję Wam za Wasze nieocenione wsparcie. Jestem wdzięczna za Waszą nieustającą troskę, pomoc, poświęcenie oraz niegasnącą miłość. Dziękuję Wam za to, że zawsze jesteście dla mnie obecni i uczestniczycie w moim życiu każdego dnia, choć dzieli nas spora odleglość. Dziękuję Wam za niekończącą się walkę o mnie. Dziękuję Wam również za to, że znacie mnie lepiej, niż ja samą siebie. Bez Was, bez Waszej dobrej rady, otuchy, jak rownież potrzebnych słow krytyki, poległabym na tej trudnej drodze.

I dedicate this work to my beloved and never forgotten Grandparents Weronika Can, Zofia Wróbel, Stanisław Can and Czesław Wróbel. 


\section{Publications}

\section{Publications:}

Müller M. and Can K. (2014) Aberrant redox homoeostasis and mitochondrial dysfunction in Rett syndrome. Biochem Soc Trans 42, 959-964.

Weller J., Kizina K. M., Can K., Bao G. and Muller M. (2014) Response properties of the genetically encoded optical $\mathrm{HO}$ sensor HyPer. Free Radic Biol Med 76C, 227-241.

Bodda C., Tantra M., Mollajew R., Arunachalam J. P., Laccone F. A., Can K., Rosenberger A., Mironov S. L., Ehrenreich H. and Mannan A. U. (2013) Mild overexpression of Mecp2 in mice causes a higher susceptibility toward seizures. Am J Pathol 183, 195-210.

\section{Oral presentations:}

Can K., Tolö J., Kügler S., Janc O.A., Müller M. (2015) Rett syndrome provokes redox imbalance already in neonatal neurons, affecting the cytosol and the mitochondria. $11^{\text {th }}$ Göttingen Meeting of German Neuroscience Society, 'Breaking News', Göttingen, Germany, o. S23-4

Can K. (2014) Importance of monitoring pH changes during redox imaging. PhD Retreat, Leipzig, Germany

Can K. (2013) Redox imbalance and oxidative stress in MeCP2 deficient neurons. Neurobiology Doctoral Students Workshop, Berlin, Germany

Can K. (2013) Dynamic recording of cellular redox status. PhD Retreat, Köln, Germany

\section{Posters:}

Can K., Tolö J., Kügler S., Kempkes B., Kolbrink B., Wagener K., Müller M. (2015) Rett syndrome provokes a cytosolic and mitochondrial redox imbalance in neonatal neurons. Encephalon, $3^{\text {rd }}$ biennial Neuroscience Conference, Göttingen, Germany, Abstract p.2.

Can K., Tolö J., Kügler S., Kempkes B., Kolbrink B., Wagener K., Müller M. (2015) Rett syndrome provokes a cytosolic and mitochondrial redox imbalance in neonatal neurons. APS Conference: Physiological Bioenergetics: From Bench to Bedside, Tampa, USA, Abstract p. 7.4.

Can K., Tolö J., Kügler S., Müller M. (2014) Aberrant redox homeostasis in RETT syndrome affects cytosol and mitochondria. Society for Neuroscience, Washington, USA, Abstract p.V21 515.17. 
Can K., Tolö J., Kügler S., Müller M. (2013) Aberrant mitochondrial redox balance and ROS generation in Rett mouse hippocampal neurons. $3^{\text {rd }}$ European Rett Syndrome Conference, Maastricht, Netherlands, Abstract p.05.

Can K., Tolö J., Kügler S., Müller M. (2013) Aberrant mitochondrial redox balance and ROS generation in Rett mouse hippocampal neurons. Neurobiology Doctoral Students Workshop, Göttingen, Germany, Abstract p.P3. 


\section{Curriculum vitae}

$\begin{array}{ll}\text { Name } & \begin{array}{l}\text { Karolina Can } \\ \text { Address }\end{array} \\ & \begin{array}{l}\text { Leibnizstr.6 } \\ \text { 37083 Göttingen }\end{array} \\ \text { Email } & \begin{array}{l}\text { karolina.can@gmail.com } \\ +4915756744842\end{array} \\ \text { Mobile } & 21.07 .1987 \\ \text { Date of birth } & \begin{array}{l}\text { Bielawa } \\ \text { polish } \\ \text { Place of birth } \\ \text { fationality }\end{array} \\ \text { Sex } & \end{array}$

Education

Since 07/2012 Ph.D Student of the DFG research Center for Nanoscale Microscopy and Molecular Physiology of the Brain (CNMPB), Göttingen

Thesis: Redox imbalance and oxidative stress in Mecp2 deficient neurons

10/2006-09.2012 Master of Science at Jagiellonian University in Cracow, Poland

Thesis: Identification of insertion site for the Mecp2 $2^{R 270 \_E G F P}$ transgene in mouse and functional characterization of Mecp2 $2^{R 270}$ mutant mouse models

11/2009-11/2011 Annual practice and Master thesis executing at the Institute of Human Genetics at Georg-August Universität Göttingen, Göttingen, Germany

09/2003-09/2006 I LO im. Jędrzeja Śniadeckiego, Dzierżoniów, Poland

Higher education entrance qualification, Matura 